\title{
Behavioral Impairment Following Gestational Exposure to Titanium Dioxide Nanomaterial Aerosols in Male and Female Rats
}

\author{
Matthew Leland Eckard \\ West Virginia University, mleckard@mix.wvu.edu
}

Follow this and additional works at: https://researchrepository.wvu.edu/etd

Part of the Experimental Analysis of Behavior Commons, and the Toxicology Commons

\section{Recommended Citation \\ Eckard, Matthew Leland, "Behavioral Impairment Following Gestational Exposure to Titanium Dioxide Nanomaterial Aerosols in Male and Female Rats" (2019). Graduate Theses, Dissertations, and Problem Reports. 7469. \\ https://researchrepository.wvu.edu/etd/7469}

This Dissertation is protected by copyright and/or related rights. It has been brought to you by the The Research Repository @ WVU with permission from the rights-holder(s). You are free to use this Dissertation in any way that is permitted by the copyright and related rights legislation that applies to your use. For other uses you must obtain permission from the rights-holder(s) directly, unless additional rights are indicated by a Creative Commons license in the record and/ or on the work itself. This Dissertation has been accepted for inclusion in WVU Graduate Theses, Dissertations, and Problem Reports collection by an authorized administrator of The Research Repository @ WVU. For more information, please contact researchrepository@mail.wvu.edu. 


\title{
Behavioral Impairment Following Gestational Exposure to Titanium Dioxide Nanomaterial Aerosols in Male and Female Rats
}

\author{
Matthew L. Eckard, M.S. \\ Dissertation submitted to the Eberly College of Arts and Sciences \\ at West Virginia University \\ In partial fulfillment of the requirement for the degree of \\ Doctor of Philosophy in Psychology \\ Karen G. Anderson, Ph.D. (Chair) \\ Michael Perone, Ph.D. \\ Steven G. Kinsey, Ph.D. \\ Cole Vonder Haar, Ph.D. \\ Timothy R. Nurkiewicz, Ph.D. \\ Department of Psychology
}

Morgantown, West Virginia

2019

Keywords: behavior, titanium dioxide, nanomaterial aerosol, rats, sex differences, reversal learning, response inhibition

Copyright 2019 Matthew L. Eckard 


\title{
ABSTRACT \\ Behavioral Impairment Following Gestational Exposure to Titanium Dioxide Nanomaterial Aerosols in Male and Female Rats
}

\author{
Matthew L. Eckard
}

Engineered nanomaterials (ENMs) are beginning to be recognized as hazardous to human and animal health. Titanium dioxide $\left(\mathrm{TiO}_{2}\right)$ is primarily used as a whitening agent in paints, plastics, and sunscreens. While relatively inert in its bulk form, nano- $\mathrm{TiO}_{2}(<100$ nanometer particle diameter) can produce prolonged inflammation and oxidative stress in target tissue. Recently, the potential for nano- $\mathrm{TiO}_{2}$ to cause neuroinflammation and damage has heightened concerns about its continued use. One important concern is that nano- $\mathrm{TiO}_{2}$, and other metal oxide ENMs, may affect fetal neurodevelopment. Accordingly, it is imperative to screen ENMs, like $\mathrm{TiO}_{2}$, for possible neurotoxicity following developmental exposures. The current set of studies assessed behavioral outcomes in male and female rat offspring following gestational exposure to nano$\mathrm{TiO}_{2}$ aerosols. Pregnant Sprague-Dawley rats were exposed to nano- $\mathrm{TiO}_{2}$ aerosols $\left(12 \mathrm{mg} / \mathrm{m}^{3}\right)$ across 6 days from gestational day (GD) 12-20 for $6 \mathrm{~h} /$ day. Testing of the offspring began at postnatal day (PND) 70. While no exposure-related deficits were detected in delayed reinforcement learning, nano- $\mathrm{TiO}_{2}$-exposed male rats showed increased perseverative error and reduced response latencies in a discrimination reversal task. Additionally, nano- $\mathrm{TiO}_{2}$-exposed male rats showed higher response rates and response inefficiency on a DRL 30-s schedule. Behavioral impairment was not evident in nano- $\mathrm{TiO}_{2}$-exposed female rats indicating potential sex-specific effects of ENM exposure. Behavioral impairment was not robust and performance in nano- $\mathrm{TiO}_{2}$-exposed male rats approximated that of controls after sufficient training. These data extend previous work by not only showing that behavioral impairment can be detected in sensitive operant behavioral measures following extremely low-level nano- $\mathrm{TiO}_{2}$ aerosol exposures, but also that males may be more susceptible than females. Together, the current study adds to the emerging literature that developmental exposure to nano- $\mathrm{TiO}_{2}$ can affect executive function in the offspring. 


\section{Acknowledgements}

The culmination of my graduate work, including this dissertation, would not have been possible without the continued support of many different people. I would like to thank my primary advisor Karen Anderson. After an extremely stressful first year at WVU, she took me on as a student my second year, and gave me just the right amount of scientific guidance and freedom to work on many different fulfilling projects. I would also like to thank my informal advisor Steve Kinsey. His attention to detail and desire to communicate science effectively have been major driving forces in my orientation to and communication of my scientific work.

In addition to Karen and Steve, the other members of my dissertation committee have my utmost thanks as well. Michael Perone sparked my interest in computer programming, which has been an invaluable tool in accomplishing all of the projects I have been fortunate enough to work on at WVU. Cole Vonder Haar taught me the utility of alternative statistical strategies and also how to effectively couple operant behavioral methods with sophisticated tissue analyses that will be critically important in my future work. Lastly, Tim Nurkiewicz was gracious enough to allow me to shadow members of his laboratory for a semester to learn about inhalation toxicology, which ultimately put me in a competitive position to secure my next academic position focusing on air pollution exposure.

To all of my friends and lab mates at WVU, my sanity could not have been composed without you.

Finally, I would like to thank my family - my mother Valerie, my father Leland, and my brother Jake. Their unconditional emotional, financial, and practical support throughout both my undergraduate and graduate work has been indispensable. They always seem to bring light to even the darkest days. 


\section{Table of Contents}

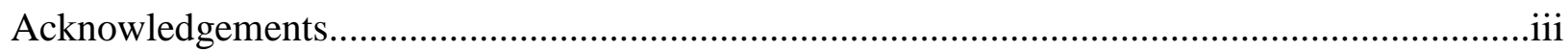

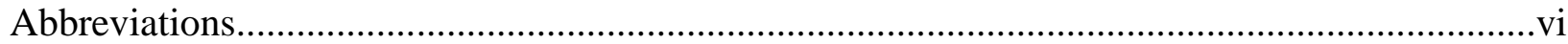

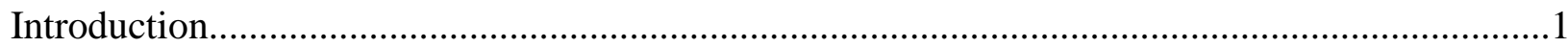

Common Exposure Routes of Engineered Nanomaterial.....................................................2

Neurological and Behavioral Effects of Direct ENM Exposure..............................................

Neurological and Behavioral Effects of Gestational/Developmental ENM Exposure............5

Exposure to Air Pollution as a Proxy for ENM...................................................................

Statement of the Problem.............................................................................................

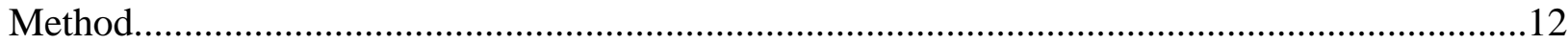

Nanomaterial Aerosol Characterization.....................................................................12

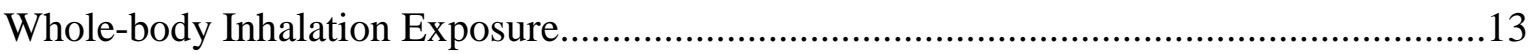

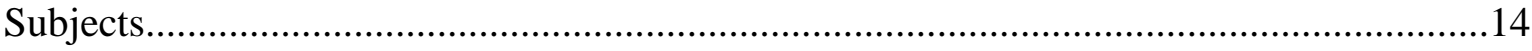

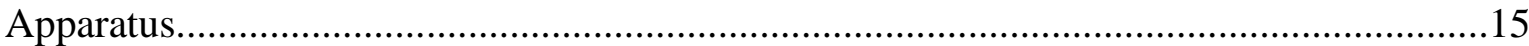

Experiment 1: Response Acquisition with Delayed Reinforcement.....................................15

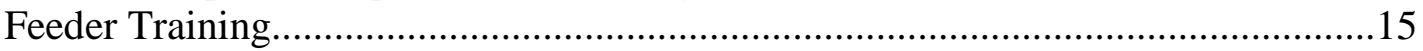

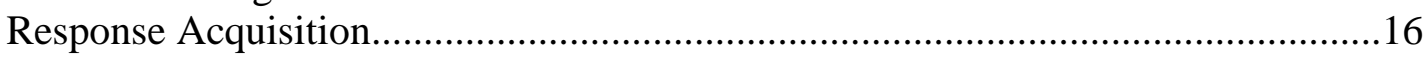

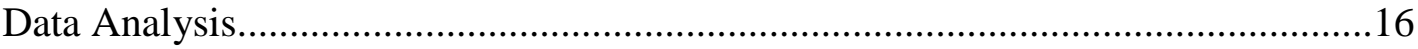

Experiment 2: Spatial Discrimination Reversal...……….............................................17

Alternating FR Training...............................................................................17

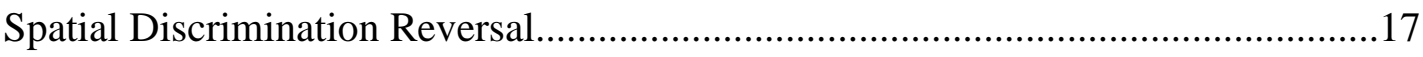

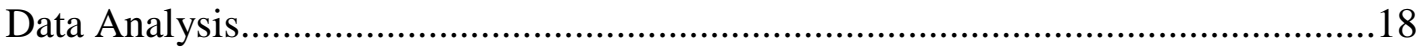

Experiment 3: Differential Reinforcement of Low Rate...................................................19

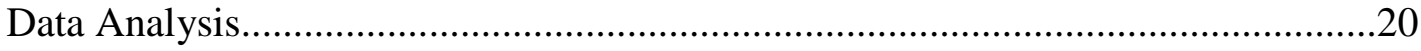

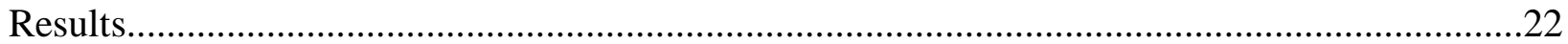

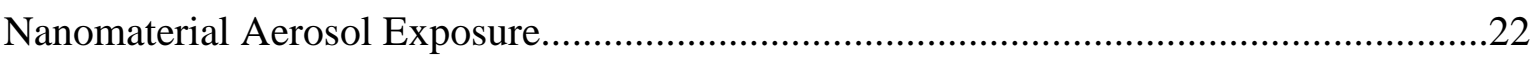

Experiment 1: Response Acquisition.......................................................................22

Experiment 2: Spatial Discrimination Reversal............................................................23

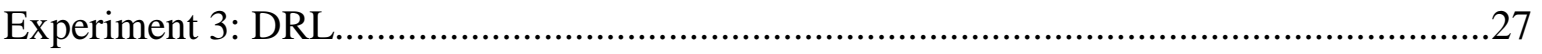

DRL 5-s schedule

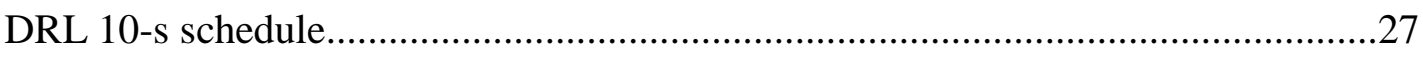

DRL 20-s schedule

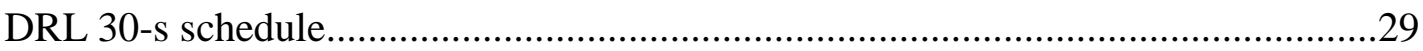

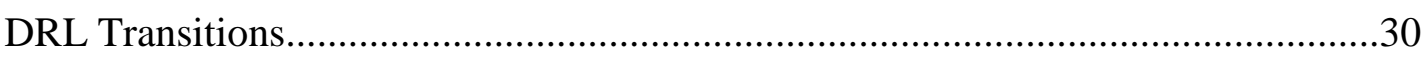

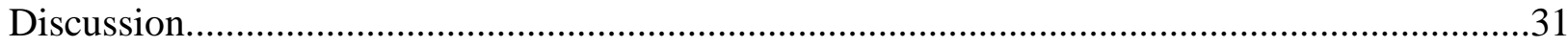

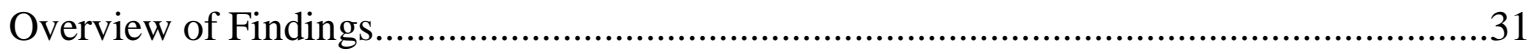

Lack of Impairment in Response Acquisition..................................................................32

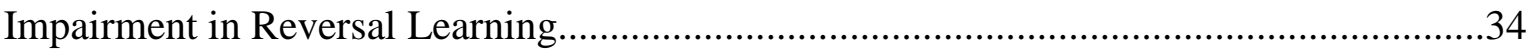

Response Inhibition Impairment........................................................................................

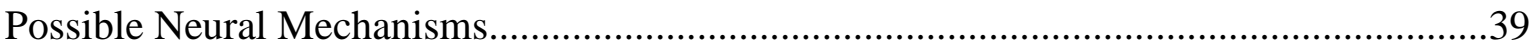

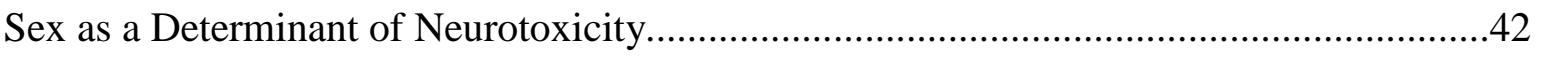

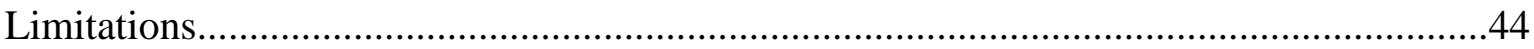

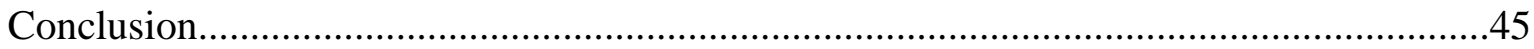

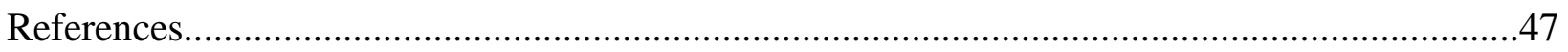




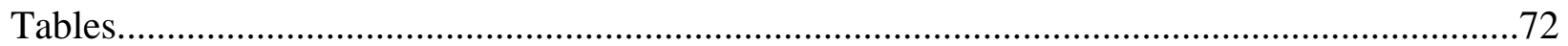

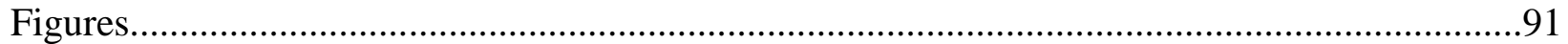

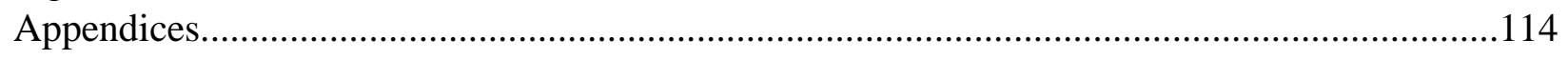




\section{Abbreviations}

ENM - engineered nanomaterial

$\mathrm{TiO} 2$ - titanium dioxide

BBB - blood-brain barrier

GFAP - glial fibrillary protein

GD - gestational day

$\mathrm{PM}$ - particulate matter

UFP - ultrafine particulate matter

DRL - differential reinforcement of low rates

ELPI - electronic low-pressure impactor

SMPS - scanning mobility particle sizer

APS - aerodynamic particle sizer

MOUDI - micro-orifice uniform deposit impactor

HPAG - high-pressure acoustical generator

PND - postnatal day

VT - variable time

FR - fixed ratio

ITI - intertrial interval

SDR - spatial discrimination reversal

ANOVA - analysis of variance

IRT - interresponse time

GSD - geometric standard deviation

PFC - prefrontal cortex

ASD - autism-spectrum disorder

ADHD - attention deficit/hyperactivity disorder

ROS - reactive oxygen species 


\section{Introduction}

Since one of its first initial descriptions (Feynman, 1960), the field of nanotechnology has been continually progressing. In 2015, it was estimated that over 1800 consumer products containing engineered nanomaterials (ENMs) were available to consumers (Vance et al., 2015). ENMs are broadly defined as anthropogenic material with particle diameters measuring < 100 nanometers (nm). Due to their small size, ENMs have physicochemical characteristics distinct from those of their bulk counterparts. For example, they have much greater surface area and reactivity than bulk material allowing for a range of special applications (Guo, Xie, \& Luo, 2014). ENMs, including various metal oxides, are used widely across medical, industrial, and consumer applications (Montazer \& Seifollahzadeh, 2011; Reimhult, 2015; Ujjain, Roy, Kumar, Singha, \& Khare, 2016). For example, titanium dioxide $\left(\mathrm{TiO}_{2}\right)$ nanomaterial is used in many applications including cleaning air and water via photocatalysis, blocking ultra-violet rays in sunscreens, and serving as white pigment in paints, foods, plastics, and toothpastes (Montazer \& Seifollahzadeh, 2011).

Despite their inherent advantages across numerous applications, extensive assessments of ENM toxicity remain warranted (Hendren, Mesnard, Dröge, \& Wiesner, 2011; Nel, Xia, Madler, \& Li, 2006). More specifically, our understanding of how ENM exposure affects neurological and behavioral outcomes are critically lacking. The extent to which gestational ENM exposure affects these outcomes is even less understood. Gestation is a critical window of brain development during which toxicant exposure can produce long-lasting neurological and behavioral deficits relative to similar exposures during adulthood (Rice \& Barone, 2000). To date, only five studies have evaluated the impacts of gestational ENM exposure on behavioral outcomes in the offspring (Cui et al., 2014; Engler-Chiurazzi et al., 2016; Hougaard et al., 2010; Jackson, Vogel, Wallin, \& Hougaard, 2011; Notter et al., 2018). Importantly, these studies differ 
on route of ENM exposure (e.g., injection, oral gavage, and inhalation), dose, and behavioral targets. Moreover, these studies did not include systematic repeated-measures designs to evaluate long-term learning processes, which is a critical component of evaluating neurobehavioral deficits following gestational neurotoxicant exposure (Cory-Slechta, 2010; Laties, 1978). The current set of experiments was designed to assess behavioral deficits in rats exposed during gestation to nano- $\mathrm{TiO}_{2}$ aerosols by maternal inhalation. Additionally, the selected nano- $\mathrm{TiO}_{2}$ exposure regimen represents are more realistic exposure relative to prior "proof-of-concept" exposure doses. Finally, behavioral assays were selected to target specific behavioral processes, which have been consistently perturbed following putative neurotoxicant exposure.

\section{Common Exposure Routes of Engineered Nanomaterial}

Due to continued industrial production of ENMs across a wide range of applications (Mueller \& Nowack, 2008; Piccinno, Gottschalk, Seeger, \& Nowack, 2012; Robichaud, Uyar, Darby, Zucker, \& Wiesner, 2009; Vance et al., 2015), it is important to consider the routes by which humans may be exposed to ENMs. The three primary exposure routes are transdermal, ingestion, and inhalation. Exposure from injection and implanted medical devices are also potential routes of exposure that should not be overlooked but are not considered primary exposure routes. Dermal exposure to ENM mostly occurs by using cosmetic products on the skin, or drug therapies designed for dermal absorption. Aerosol and lotion-based sunscreens are common ENM-containing products in which nano- $\mathrm{TiO}_{2}$ and zinc oxide are effective at reflecting ultraviolet radiation (Montazer \& Seifollahzadeh, 2011). While these materials are relatively ineffective at penetrating intact or damaged skin,hair follicles represent a potential portal of entry for ENMs applied to the skin (Monteiro-Riviere et al., 2011). Ingestion of ENMs primarily occurs through their presence in various food products (Pathakoti, Manubolu, \& Hwang, 2017; 
Srinivas et al., 2010) or by secondary mucosal clearance from the lung of inhaled particles that are subsequently swallowed (Gaté et al., 2017). ENMs are often included in foods and food packaging for antimicrobial purposes to increase shelf life or to increase the solubility of the foods (Pathakoti et al., 2017). After ingestion, ENMs have the potential to translocate to the systemic circulation from the digestive tract and become distributed throughout the body (Jani, Halbert, Langridge, \& Florence, 1990). However, intestinal absorption of ENM tends to be negligible (Kreyling et al., 2017).

Inhalation represents the most physiologically relevant exposure route for ENMs in humans. This is largely based on the relative ease with which airborne particles can enter the lung and the inefficiency of particle clearance from the lung following inhalation. Macrophage phagocytosis is the predominant clearance mechanism for inhaled particulate matter entering the alveoli of the lung (Warheit, Overby, George, \& Brody, 1988), which is the primary area in which nano-sized particles deposit (International Commission on Radiological Protection, 1994). Relative to micron-sized particles, which show approximately $80 \%$ clearance $24 \mathrm{~h}$ after inhalation, only about $0.1 \%$ of nano-sized particles are cleared $24 \mathrm{~h}$ after inhalation (Geiser \& Kreyling, 2010; Geiser et al., 2008). Thus, once inhaled, ENMs tend to remain in the lung longer relative to larger particles. For other routes of exposure, ENMs either do not penetrate easily (dermal) or are excreted more efficiently after entering the body (ingestion). Inhalation of ENM, or ultrafine particulate matter generally, can occur via a wide range of sources including ambient air (Valavanidis \& Fiotakis, 2008) during the production, transportation, and storage of bulk quantities of ENMs (e.g., nano-TiO 2 and nano-silver) (Mueller \& Nowack, 2008; Robichaud et al., 2009), and by inhalation of consumer spray products containing ENMs (Losert et al., 2014; Park et al., 2017). In addition to its physiological relevance, ENM inhalation is perhaps the most 
developed area of ENM toxicity stemming from earlier work on ultrafine particulate matter in air pollution (Oberdörster et al., 2000; Oberdörster, Oberdörster, \& Oberdörster, 2005; Valavanidis $\&$ Fiotakis, 2008). One consequence of the lack of particle clearance from the lung is that it increases the probability that ENM particles will translocate from the lung to systemic circulation eventually becoming deposited in secondary organs leading to systemic inflammation (Geiser et al., 2008; Semmler et al., 2004). Indeed, deposits of inhaled or intratracheally instilled ENMs (e.g., elemental carbon, iridium, silver, uranium, $\mathrm{TiO}_{2}$, manganese oxide) have been found in the liver, spleen, kidneys, and the brain of rats and mice, among other areas (Elder et al., 2006; Oberdörster et al., 2004; Petitot et al., 2013; Pujalté, Dieme, Haddad, Serventi, \& Bouchard, 2017; Semmler et al., 2004; Takenaka et al., 2001; Wang et al., 2008). Thus, there is potential for ENMs to produce a wide range of toxicological effects following inhalation.

\section{Neurological and Behavioral Effects of Direct ENM Exposure}

Both direct and gestational exposure to ENMs can be neurotoxic in animal models (see Bencsik, Lestaevel, \& Guseva Canu, 2018, Cupaioli, Zucca, Boraschi, \& Zecca, 2014, and Oberdörster, Elder, \& Rinderknecht, 2009 for reviews). After direct exposure, ENM particles can gain entry to the brain by either crossing the blood-brain barrier (BBB) or by anterograde axonal transport via the olfactory nerve pathway during inhalation (e.g., De Lorenzo, 1970). Exposure to ENMs through the airways via inhalation or intranasal instillation in rats produces ENM deposits in the olfactory bulb (Oberdörster et al., 2004) with lower but significant concentrations in the striatum, frontal cortex, cerebellum, and the hippocampus (Elder et al., 2006, Wang et al., 2008a; Wang et al., 2008b). After contacting target tissue, ENM can induce neuroinflammation. For example, intragastric administration of high-dose nano- $\mathrm{TiO}_{2}$ (i.e., $50-200 \mathrm{mg} / \mathrm{kg}$ ) increased expression of glial fibrillary acidic protein (GFAP) indicating astrocyte activation (Grissa et al., 
2016). Although these previously mentioned studies did not assess behavioral deficits following exposure, large doses of nano- $\mathrm{TiO}_{2}$ (i.e., $5-50 \mathrm{mg} / \mathrm{kg}$, p.o.) administered repeatedly (i.e., 60-90 days) produce spatial memory deficits in Y-maze and Morris water maze tests in mice (Hu et al., 2010; Ze et al., 2014). In the only study assessing deficits in operant behavior, a single injection of nano- $\mathrm{TiO}_{2}$ suspensions (approximately $20 \mu \mathrm{g}$ ) into the lateral ventricles of rats produced spatial discrimination deficits in a task requiring alternating responses across two levers for up to seven days post-injection (Kim et al., 2013). These behavioral deficits are collectively associated with dose-dependent decreases in dopamine and serotonin either in the whole brain (Hu et al., 2010) or striatum and frontal cortex of mice (Zhang et al., 2011). Thus, behavioral and neurological outcomes are sensitive to direct ENM exposure; however, the majority of these studies used doses that are extremely high, potentially leading to pulmonary overload if they were to be translated into an inhalation paradigm (Warheit, 2013), which perhaps limits their generality. Nonetheless, one primary conclusion to be drawn from direct ENM exposure studies is that ENMs are not benign and represent a realistic threat to neurological health following exposure.

\section{Neurological and Behavioral Effects of Gestational/Developmental ENM Exposure}

Given the observed effects of direct ENM exposure, it follows that exposure during gestation may produce more profound effects. It is widely accepted that gestation represents a critical developmental window in which toxicant exposure can negatively affect neural development and produce long-lasting deficits in executive function (Rice \& Barone, 2000). In rodents, brain formation begins at approximately gestational day (GD) 7 and, in humans, around GD 21 (Rice \& Barone, 2000; Sadler, 2005). During these early stages, healthy brain development can be compromised by an aversive gestational environment via toxicant exposure 
(Rice \& Barone, 2000; Wells et al., 2005). While an understanding of neurological and behavioral outcomes following gestational ENM exposure are still limited, several studies indicate that it is associated with alterations in neurotransmitter systems, inflammatory responses, and behavioral deficits in short-term behavioral assays. For example, systemic administration of nano- $\mathrm{TiO}_{2}$ during gestation produced titanium deposits in the frontal and temporal lobes of mouse offspring up to six weeks after exposure (Takeda et al., 2009). Similar gestational nano- $\mathrm{TiO}_{2}$ exposures in mice also produced alterations in gene expression related to brain development (Shimizu et al., 2009; Umezawa, Tainaka, Kawashima, Shimizu, \& Takeda, 2012) and increased dopamine levels in the prefrontal cortex and striatum (Takahashi, Mizuo, Shinkai, Oshio, \& Takeda, 2010).

As changes in the brain generally carry little meaning without concomitant changes in behavior (Krakauer, Ghazanfar, Gomez-Marin, MacIver, \& Poeppel, 2017), detection of behavioral impairment following gestational ENM exposure is a crucial step in establishing ENM as a developmental neurotoxicant. To date, the few studies reporting behavioral deficits following gestational ENM exposure center around increased depressive-like behavior and deficits in spatial memory and social interaction in rodents (Cui et al., 2014; Engler-Chiurazzi et al., 2016; Fu et al., 2018; Hougaard et al., 2010; Jackson et al., 2011; Mohammadipour et al., 2014; Notter et al., 2018). As might be expected, robust deficits are typically detected after extremely high exposures (e.g., $100 \mathrm{mg} / \mathrm{kg}$ nano- $\mathrm{TiO}_{2}$ orally throughout gestation; Mohammadipour et al., 2014) whereas more subtle deficits are found using realistic exposure routes and doses (e.g., $350 \mu \mathrm{g}$ nano- $\mathrm{TiO}_{2}$ via inhalation throughout gestation; Engler-Chiurazzi et al., 2016). The findings of Engler-Chiurazzi and colleagues (2016) are particularly interesting because longer latencies to locate a platform submerged in water and greater working memory 
errors in a radial arm maze were detected in male rat offspring following maternal inhalation of nano- $\mathrm{TiO}_{2}$ at an extremely low dose (i.e., $350 \mu \mathrm{g}$ total throughout gestation) relative to other studies using unrealistically high exposures. Therefore, there is reason to explore other, longterm behavioral deficits following low-dose maternal inhalation exposures rather than continuing with high-dose, proof-of-concept exposure regimens and simplistic behavioral targets.

\section{Exposure to Air Pollution as a Proxy for ENM}

Despite this promising avenue for future research, no long-term studies of behavior following low-dose gestational exposures similar to Engler-Chiurazzi et al. (2016) have been conducted. This paucity of research not only creates a substantial knowledge gap regarding neurodevelopmental effects of low-dose gestational ENM exposure, but also makes it difficult to select repeated-measures behavioral targets for initial study. However, studies of prenatal and neonatal exposure to fine and ultrafine particulate matter (PM) in ambient air pollution provide some basis for selecting behavioral targets. The constituents of air pollution include course, fine, and ultrafine PM, the latter of which is similar in size to nanomaterial (i.e., approximately 100 $\mathrm{nm}$ in diameter). Ambient air pollution is not only associated with increased mortality (Di, Dai, et al., 2017; Di, Wang, et al., 2017; World Health Organization, 2017), but also with deficits in learning outcomes in children exposed during gestation to air pollution (Chiu et al., 2017; Guxens et al., 2018; Payne-Sturges et al., 2019; Sunyer \& Dadvand, 2019; Sunyer et al., 2015; Volk, Lurmann, Penfold, Hertz-Picciotto, \& McConnell, 2013). Additionally, ambient air pollution is correlated with heightened incidences of attention-deficit/hyperactivity disorder (ADHD) (Min \& Min, 2017), a neurodevelopmental disorder characterized by inattention and impulsivity. For example, fine particle concentration in air pollution during pregnancy was associated with thinner frontal lobe cortices in children and higher response inhibition errors on a 
response set task (Guxens et al., 2018). Deficits in children's executive function also correlate with proximity to high-density air pollution such that children attending schools closer to hightraffic areas have decreased performance in working memory tasks relative to children in lowertraffic areas (Sunyer et al., 2015). Furthermore, there appear to be sex-specific effects of air pollution in which IQ and reaction times were lower and higher, respectively, in male children whereas memory deficits were more commonly detected in female children (Chiu et al., 2017). Importantly, exposures during mid-to-late pregnancy had stronger associations with behavioral outcomes than exposures during early pregnancy (Chiu et al., 2017).

Animal models of gestational and neonatal exposure to ambient air pollution also show consistent deficits in repeated-measures evaluations of executive function that overlap with behavioral deficits found in human populations (Allen et al., 2017(a), 2017(b)). In mice, neonatal exposure to concentrated ultrafine air pollution (UFP), which aligns with the third trimester of human gestation (Rice \& Barone, 2000), produces deficits in operant behavior tasks requiring response inhibition and behavioral flexibility to earn food rewards (Allen et al., 2013; CorySlechta, Allen, Conrad, Marvin, \& Sobolewski, 2018; Morris-Schaffer et al., 2018). One of the tasks used by Cory-Slechta et al. (2018) was a differential-reinforcement-of-low-rates (DRL) schedule of reinforcement. In this task, reinforcers are earned if responses are spaced by some minimum interval (Zimmerman \& Schuster, 1962). If a response occurs too early, no reinforcer is provided, the response timer is reset to zero, and responding must be withheld until the criterion time elapses again. Thus, the subject must learn to inhibit responding appropriately. Following neonatal UFP exposure, adult mice earned fewer reinforcers and lever pressed more frequently than air-exposed mice across successive transitions from short to long DRL schedules without systematic exposure-related deficits within a given DRL schedule (Cory-Slechta et al., 
2018). Similarly, rats chronically exposed to UFP for the first 25 weeks after birth earned fewer reinforcers on short DRL schedules relative to controls with these differences subsiding on longer DRL schedules (Woodward et al., 2018). Furthermore, these deficits in DRL responding were observed more consistently in male mice than in females (Cory-Slechta et al., 2018), but similar data on female rats are lacking (Woodward et al., 2018).

In addition to response inhibition deficits, behavioral flexibility is also impacted by neonatal UFP exposure. In behavioral flexibility paradigms, a discrimination typically is trained between two stimuli that differ in visual or spatial dimensions such that responding to one stimulus is reinforced $(\mathrm{S}+)$ while responding to the other is not reinforced (S-) (Dias, Robbins, \& Roberts, 1996). Then, the contingencies for reinforcement are manipulated such that the $\mathrm{S}+$ and S- are reversed or a discrimination for another stimulus dimension is reinforced (e.g., stimulus shape vs. spatial location). The former is usually termed a discrimination reversal or intradimensional shift whereas the latter is termed an extradimensional shift. Morris-Schaffer et al. (2018) reported that male, but not female, mice exposed to UFP had increased discrimination errors during initial training relative to air-exposed controls. Interestingly, no exposure-related deficits were detected shortly after a discrimination reversal occurred. However, when the informational cue changed from lever position (i.e., left vs. right) to a randomly alternating light above each lever (i.e., extradimensional shift), UFP-exposed males displayed increased response errors across sessions relative to controls with no differences detected in female mice (MorrisSchaffer et al., 2018). These deficits in response inhibition and behavioral flexibility following UFP exposure also align with previous work using similar behavioral paradigms to establish lead, methylmercury, and other substances as developmental neurotoxicants (Bushnell, 1988; Paletz, Craig-Schmidt, \& Newland, 2006; Paletz, Day, Craig-Schmidt, \& Newland, 2007; Reed, 
Paletz, \& Newland, 2006; Rice, 1992, 1998; Rice \& Gilbert, 1985; Sable, Eubig, Powers, Wang, \& Schantz, 2009; Widholm et al., 2001). Thus, these tasks may also be useful in uncovering possible deficits caused by gestational ENM exposure.

Disruptions in brain morphology, neuroimmune response, and neurochemistry also align with behavioral outcomes following gestational and neonatal UFP exposures. Mice exposed to UFP during the neonatal window showed enlarged ventricles, microglia and astrocyte activation in the hippocampus, reduced corpus callosum myelination, and increased dopamine, norepinephrine, and glutamate in the striatum (Allen, Liu, Pelkowski, et al., 2014a; Allen, Liu, Weston, Conrad, et al., 2014b; Allen, Liu, Weston, Prince, et al., 2014c; Allen et al., 2017). Furthermore, neonatal UFP exposure increased glutamate excitotoxicity and microglia activation in the hippocampus of mice (Allen et al., 2017; Allen, Liu, Weston, Conrad, et al., 2014b). These neurological effects are also largely male-specific, which mirror observed behavioral deficits. Gestational exposure to UFP also resulted in enlarged ventricles, reduced hippocampal volume, and increased corpus callosum myelination; although, it is thought that this latter effect is perhaps a compensatory response (Klocke et al., 2017, 2018). Interestingly, sex-specific differences in neurological outcomes were minimized following gestational UFP exposure (Klocke et al., 2017). However, behavioral outcomes following gestational UFP exposure remain largely unknown.

To the extent that gestational UFP exposure mimics consequences of ENM exposures, the current literature on neurodevelopmental consequences of UFP justifies similar investigations following ENM exposure. Moreover, there appear to be similar mechanisms of neural insult following exposure to either UFP or ENM such that monoamine systems are dysregulated, inflammatory markers are upregulated, and brain regions critical for memory and decision- 
making are impacted (Allen, Liu, Weston, Conrad, et al., 2014b; Cui et al., 2014; Klocke et al., 2018; Mohammadipour et al., 2014). Additionally, selection of similar behavioral targets for ENM neurotoxicity allows some degree of comparison to other, more established neurotoxicants like heavy metals. Finally, this inductive approach beginning with standard operant behavioral paradigms will provide a basis for exploring additional behavioral phenotypes that may manifest following gestational ENM exposure, which may be undetected by focusing on behavioral inhibition and flexibility targets specifically (e.g., fine motor function and choice).

\section{Statement of the Problem}

Given the behavioral and neurological outcomes following gestational/neonatal ultrafine air pollution exposure, it is unclear if gestational ENM aerosol exposure will produce similar outcomes. To date, such comparisons are the closest approximation to what might occur following gestational ENM exposure. With current data suggesting that low-level gestational exposure to nano- $\mathrm{TiO}_{2}$ aerosols produces detectable behavioral deficits in male rat offspring using short-term, relatively simplistic behavioral tasks (Engler-Chiurazzi et al., 2016), use of more complex, long-term tasks to probe behavioral phenotypes like those reported following air pollution exposures is warranted. Specifically, response inhibition, behavioral flexibility, and operant learning in general are commonly used behavioral targets to establish a given environmental agent as a neurotoxicant, including developmental exposure to ultrafine or nanosized aerosols (Cory-Slechta et al., 2018; Klocke et al., 2018; Morris-Schaffer et al., 2018). These assessments are necessary in the continued effort of ENM hazard identification such that the safe designs of ENMs can progress.

The present series of experiments was aimed at elucidating behavioral impacts of gestational exposure to nano- $\mathrm{TiO}_{2}$ aerosols. Nano- $\mathrm{TiO}_{2}$ was used because it is one of the most 
commonly used commercial ENMs with estimated yearly production of approximately 40,000 metric tons in 2008, which is expected to continually rise as ENMs gain popularity (Hendren et al., 2011; Robichaud et al., 2009). The current approach will use methods commonly implemented in the characterization of neurotoxicants to assess lever-press acquisition in the context of delayed reinforcement (Experiment 1), perseveration and behavioral flexibility (Experiment 2), and response inhibition (Experiment 3). Additionally, given the male-biased toxicity of UFP exposures in mice and higher susceptibility of male rodents to neurotoxic insult generally (Kern et al., 2017), male and female rat offspring were included in the current study. It is hypothesized that gestational exposure to nano- $\mathrm{TiO}_{2}$ aerosols will result in 1) impaired response acquisition, 2) increased errors in a discrimination reversal task, 3) inefficient responding across a series of DRL schedules, and 4) these impairments will be largely specific to male rats.

\section{Method}

\section{Nanomaterial aerosol characterization}

Nano- $\mathrm{TiO}_{2} \mathrm{P} 25$ powder was obtained from Evonik (Aeroxide $\mathrm{TiO}_{2}$, Parsippany, NJ). This powder is a mixture containing anatase $(80 \%)$ and rutile $(20 \%) \mathrm{TiO}_{2}$, with a primary particle size of $21 \mathrm{~nm}$, a surface area of $48.08 \mathrm{~m}^{2} / \mathrm{g}$, and a Zeta potential of $-56.6 \mathrm{mV}$ (Stapleton et al., 2018). The $\mathrm{TiO}_{2}$ powder was stored in a glass desiccator to maintain dryness for aerosolizing. Aerosol size distributions were measured during aerosol exposures while $\mathrm{TiO}_{2}$ mass concentration was maintained at the target concentration of approximately $12 \mathrm{mg} / \mathrm{m}^{3} \mathrm{using}$ : 1) a high-resolution electrical low-pressure impactor (ELPI+; Dekati, Tampere, Finland), 2) a scanning mobility particle sizer (SMPS 3938; TSI, Inc., St. Paul, MN), 3) an aerodynamic particle sizer (APS 3321; 
TSI Inc., St. Paul, MN) and 4) a nano micro-orifice uniform deposit impactor (MOUDI 115R, MSP Corp, Shoreview, MN).

\section{Whole-body inhalation exposure}

Inhalation exposures were conducted using a high-pressure acoustical generator (HPAG, IEStechno, Morgantown, WV) to produce nano- $\mathrm{TiO}_{2}$ aerosols as previously described (Abukabda et al., 2019; See Appendix A for a schematic of the exposure system). After leaving the acoustical generator, the aerosols were fed through a Venturi pump (JS-60 M, Vaccon, Medway, MA) to further deagglomerate the particles prior to entering the exposure chamber. A personal DataRAM (pDR-1500; Thermo Fisher Environmental Instruments, Inc., Franklin, MA) was used to sample the exposure chamber air in real-time, and a software controller automatically adjusted the acoustic energy to maintain a constant aerosol mass concentration (12 $\mathrm{mg} / \mathrm{m}^{3}$ ) during exposures. Gravimetric measurements were conducted using Teflon filters in the breathing zone of the animals (sample flow $=0.35 \mathrm{~L} / \mathrm{min}$ ). The results of the gravimetric measurements were used to report the average aerosol mass concentrations for each day of exposure. Sham controls were exposed to HEPA-filtered air, but all other chamber conditions (i.e., temperature, humidity) were held constant.

Four pregnant adult female Sprague-Dawley rats (Hilltop Laboratories; Scottsdale, PA) were used to produce litters following inhalation exposures (two nano- $\mathrm{TiO}_{2}$; two air control). Rats were housed in AAALAC-approved facilities with ad libitum access to food and water. Female rats were monitored for estrus, at which time each female rat was placed with an individual male rat. Female rats subsequently were given a vaginal smear every $12 \mathrm{~h}$ to verify mating via the presence of sperm. Exposures began on GD 11 to allow for uterine implantation of the embryo (Stapleton et al., 2013). Pregnant rats were exposed to a target concentration of 12 
$\mathrm{mg} / \mathrm{m}^{3}$ for $6 \mathrm{~h} /$ day for six days, which was chosen based on previous studies showing compromised cardiovascular health and behavioral deficits in rats following similar exposures (Engler-Chiurazzi et al., 2016; Stapleton et al., 2018; Stapleton et al., 2013). Daily total lung dose of nano- $\mathrm{TiO}_{2}$ aerosols was estimated by accounting for the mass proportion of particles deposited in the rat lung (10\%), the average volume of air breathed in by a rat per minute $(208.33$ $\mathrm{cc})$, the $\mathrm{TiO}_{2}$ mass concentration $\left(12 \mathrm{mg} / \mathrm{m}^{3}\right)$, and the exposure duration in minutes (360 min) (Yi et al., 2013). The cumulative lung dose of the exposure paradigm (6 h/day, 6 days) was estimated to be approximately $525 \mu \mathrm{g}$ (Abukabda et al., 2019). The final exposure occurred approximately $48 \mathrm{~h}$ prior to pup delivery.

\section{Subjects}

Twenty-nine adult male and female Sprague-Dawley rats (Male = 15 nano- $\mathrm{TiO}_{2}=7$; control $=8] ;$ Female $=14\left[\right.$ nano- $\mathrm{TiO}_{2}=6 ;$ control $\left.\left.=8\right]\right)$ across the four litters $\left(\right.$ two nano- $\mathrm{TiO}_{2} ;$ two sham control) were used as subjects. After weaning at PND 21, rats were pair-housed within exposure groups and sex in a temperature- and humidity-controlled $\left(72^{\circ} \mathrm{F}, 60 \%\right)$ vivarium operating on a reverse 12:12 h dark/light cycle. Until PND 60, food and water were freely available in the home cage. Starting on PND 60, unless otherwise specified, food was restricted to $12-15 \mathrm{~g}$ of rat chow per rat per day with water freely available. Rats received this daily allotment of food 30 minutes after daily testing resulting in approximately $22 \mathrm{~h}$ of food restriction prior to sessions. Experimental sessions were conducted five (Experiment 3) or six (Experiment 2) days per week at approximately the same time each day during the rats' dark cycle. Additionally, experimental sessions for male and female rats were always conducted in separate operant-conditioning chambers. Rats were maintained in accordance with National 
Institutes of Health guidelines for Care and Use of Laboratory Animals, and the West Virginia University Animal Care and Use Committee approved all experimental procedures.

\section{Apparatus}

Eight standard operant-conditioning chambers for rats were used for data collection, each enclosed in a sound-attenuating box (Med Associates, VT). Each chamber contained a working area of $30.5 \mathrm{~cm} \mathrm{X} 24.5 \mathrm{~cm} \mathrm{X} 21.0 \mathrm{~cm}$, a grid floor, and a $45-\mathrm{mg}$ pellet dispenser with a pellet receptacle centered between two retractable response levers. Levers were spaced $11.5 \mathrm{~cm}$ apart from each other and required at least $0.25 \mathrm{~N}$ of force for a response to be recorded. The levers were $4.8 \mathrm{~cm}$ wide, protruded $1.9 \mathrm{~cm}$ into the chamber, and were elevated $8 \mathrm{~cm}$ from the grid floor. One $28-\mathrm{V}$ stimulus light, with a $2.5 \mathrm{~cm}$ diameter, was positioned approximately $7 \mathrm{~cm}$ above each lever. A $28-\mathrm{V}$ houselight was positioned on the wall opposite the working area along with a ventilation fan to circulate air and mask extraneous noise. Experimental events and data collection were controlled by a desktop computer in an adjacent room using Med-PC software. (Med Associates, VT).

\section{Experiment 1: Response Acquisition with Delayed Reinforcement}

The following experimental protocol was adapted from Anderson and Elcoro (2007).

Feeder Training. Prior to the response-acquisition session, feeder training occurred on PND 70. Subjects were food restricted for $22 \mathrm{~h}$ prior to feeder training. The start of the session was signaled by the illumination of the houselight. During this session, response-independent food pellets were delivered to the food receptacle according to a variable-time (VT) 60-s schedule of reinforcement (Fleshler \& Hoffman, 1962). On this schedule, pellets were delivered every $60 \mathrm{~s}$ on average, independent of responding. A 0.5 -s flashing of the houselight signaled pellet delivery. Both response levers were retracted during feeder training. Feeder training 
terminated after 60 pellets were delivered in total. At the end of feeder training, food receptacles were checked to ensure all pellets were consumed. All rats consumed all food pellets.

Response Acquisition. The 8-h response-acquisition session on PND 72 occurred approximately $48 \mathrm{~h}$ after feeder training. Session start time was chosen such that all subjects were food-restricted for approximately $36 \mathrm{hr}$. After a 5 -min blackout, the acquisition session was initiated via illumination of the houselight and insertion of both response levers.

During this session, both response levers were extended into the chamber but only one lever was active for a given subject counterbalanced across and within groups. That is, the left lever was active for half the subjects and the right lever was active for the other half. Responses on the active lever were reinforced according to a tandem fixed ratio (FR) 1, fixed-time (FT) 20s schedule of reinforcement. On this schedule, an active lever press began a 20-s delay, at the end of which a pellet was delivered. This delay was not signaled and was non-resetting (Anderson \& Elcoro, 2007). Active lever presses during the delay were recorded but had no other programmed consequences. Likewise, lever presses on the inactive lever throughout the session were recorded but had no other programmed consequences.

Data Analysis. The primary dependent measures were pellets earned, active lever presses, inactive lever presses, and obtained delay (i.e., the average time between the last active lever press and the next pellet delivery) (Anderson \& Elcoro, 2007). Lever-press acquisition was also characterized qualitatively using cumulative response records (see Appendix B). Data from all experiments were analyzed using Statistica software or the R statistical package (http://www.r-project.org/). Variables were transformed as appropriate using the Box-Cox method such that each measure approximated a normal distribution (Box \& Cox, 1964). Specifically, $\operatorname{logarithmic}(\log 10(\mathrm{x}))$ transformations were applied to pellets earned, active 
presses, and inactive presses whereas cubic $\left(\mathrm{x}^{\wedge} 3\right)$ transformations were applied to obtained delay. A 2 (exposure) x 2 (sex) mixed analysis of variance (ANOVA) was used for data analysis. Significant interactions were followed by Tukey's Honestly Significant Differences post hoc test. Outcomes were considered significant if $\mathrm{p}<.05$.

\section{Experiment 2: Spatial Discrimination Reversal}

The following experimental protocol is adapted from Paletz et al. (2007) with modification.

Alternating Fixed-Ratio (FR) Training. Following Experiment 1, responding was established on both levers using an alternating FR procedure. During alternating FR sessions, either the left or right lever was extended at any given time. A single press on the extended lever produced one food pellet followed by a 10-s intertrial interval (ITI). If a lever press did occur within $15 \mathrm{~s}$ of lever extension, no pellet was delivered and the ITI began. During the ITI, the levers were retracted, and the chamber was darkened. The extended lever alternated sides (left vs. right) after every five pellet deliveries. Alternating FR sessions terminated after 60 pellets were delivered resulting in 30 total presses on each lever. This phase was in effect for three sessions.

Spatial Discrimination Reversal (SDR). Following alternating FR training, the SDR procedure began at approximately PND 75 for all rats. During SDR sessions, both levers were extended into the chamber with one lever designated as "correct" and the other lever as “incorrect." A single press on the correct lever (left or right, counterbalanced across subjects) produced one food pellet followed by a 10-s ITI, and a press on the incorrect lever ended the trial and initiated the ITI. Again, if no response occurred within $15 \mathrm{~s}$ of lever extension, the trial ended and the ITI began. The lever designated as correct remained constant throughout each session. Sessions terminated following 60 total trials. The criterion for a discrimination reversal 
was three consecutive sessions with at least 51 correct responses out of 60 total trials $(85 \%$ correct; Paletz et al., 2007). One exception to this was if the third consecutive session at criterion occurred on a Saturday. In this case, a fourth session would occur on the following Monday to ensure that the current discrimination was not disrupted by the Sunday break. This extra session occurred nine times total in both the nano- $\mathrm{TiO}_{2}$ and control groups. Upon reaching criterion, the lever designated as correct switched to the alternative lever, which was not signaled. Four total discrimination reversals occurred.

Data Analysis. Several dependent measures were calculated or recorded to characterize SDR performance. These measures included sessions to criterion - numbers of sessions required to reach the first session with an $85 \%$ accuracy criterion for the original discrimination and each reversal, errors - trials ending after an incorrect lever press for each reversal, correct:stay ratio the proportion of trials with a correct lever press that were also followed by a correct lever press, incorrect:shift ratio - the proportion of trials with an incorrect lever press that were followed by a correct lever press, and response latency - the time between trial initiation and a lever press. Various aspects of incorrect response patterns were also assessed including error runs - the number of incorrect lever press strings during a session, first-run error - the number of errors in the first error run of the session, and error run length - the number of incorrect lever presses per "run" (Paletz et al., 2007). For example, the following represents a string of trial outcomes during a reversal session: $\mathrm{C}, \mathrm{C}, \mathrm{I}, \mathrm{I}, \mathrm{I}, \mathrm{O}, \mathrm{C}$, I with $\mathrm{C}=$ correct, $\mathrm{I}=$ incorrect, and $\mathrm{O}=$ omission. In this example, there are two error runs each containing three errors and one error, respectively. The first-run error would be " 3 " and the error run length be " 2 ." Due to logistical issues, two control female rats only completed the first reversal. Thus, those subjects were removed from all analyses. 
All data from Experiment 2 except sessions-to-criterion for the original discrimination were analyzed using linear mixed-effects regression. Mixed-effects regression is a statistical tool being more commonly used by neuroscientists and behavioral researchers, and performs more optimally than ANOVA in accounting for individual subject variance across repeated observations (Boisgontier \& Cheval, 2016; Fox, 2018). Because learning the original discrimination is arguably a separate process from learning a new spatial location after a reversal, sessions-to-criterion for the original discrimination were analyzed separately using independent-samples $t$ tests. For mixed-effects regression analyses logarithmic transformations $(\log 10(\mathrm{x}))$ were applied to sessions to criterion, incorrect responses, and incorrect:shift ratios, and cubic transformations $\left(\mathrm{x}^{\wedge} 3\right)$ were applied to correct:stay ratios. All regression models allowed both intercept and slope to vary. Analyses were split a priori by sex and between the day that each reversal occurred (Session 1) and the day following each reversal (Session 2). This latter analysis allowed for an evaluation of learning across reversals after both "acute" exposure to the discrimination reversal and after having experienced each reversal for one session. Responding during the first three sessions of Reversal 1 and Reversal 2 was also analyzed to assess possible behavioral impairment within the reversals that produced most detected deficits.

\section{Experiment 3: Differential reinforcement of low rate (DRL)}

Experiment 3 began approximately 60 days following completion of the final reversal in Experiment 2. During this 60-day break, all rats remained in the colony room with free access to water and the same daily ration of food. Rats began training on the DRL 5-s schedule at approximately 6 months of age. On this schedule, responses with interresponse times (IRTs) the time between two responses - shorter than the required 5-s interval reset the IRT timer. Only responses with IRTs greater than or equal to $5 \mathrm{~s}$ were reinforced. After a 5-min blackout, DRL 
sessions were initiated by illumination of the houselight and extension of either the left or right lever (counterbalanced across subjects). Session duration was set at $60 \mathrm{~min}$. Across a preset number of sessions, the DRL schedule value increased. The DRL 5-s schedule was in effect for 10 sessions, the DRL 10- and 20-s schedules for 15 sessions, and the final DRL 30-s schedule for 30 sessions. The maximum number of pellets allowed per session was initially set at 150 for all sessions of DRL $5 \mathrm{~s}$ and the first five sessions of DRL $10 \mathrm{~s}$. Thereafter, the maximum number of pellets was set at 60 . Two female $\mathrm{TiO}_{2}$-exposed rats were excluded from all DRL analyses due to compromised health - one for progressive development of ataxia and the other for an abdominal tumor.

Data Analysis. To characterize DRL performance, the following dependent measures were calculated or recorded: reinforcement rate - reinforcers earned per minute during each session, response rate - lever presses per minute during each session, burst responses - the number of lever presses that occur less than $2 \mathrm{~s}$ after the previous lever press (IRT $<2 \mathrm{~s}$ ) per session, efficiency ratio - the ratio of nonreinforced:reinforced lever presses, median IRT - the median IRT during each session, and modal IRT - the rightward peak in IRTs as indicated by IRT distributions from each session, as outlined in Appendix C. To clarify IRT measures, median IRT is a measure of general pauses in responding whereas modal IRT is more specific to the timing of the DRL interval.

Similar to Experiment 2, data from Experiment 3 were stratified by Sex, and analyzed using either linear/nonlinear mixed-effects regression or repeated measures ANOVA. For all linear regressions, variables were transformed as appropriate using the Box-Cox method. Specifically, logarithmic $(\log 10(\mathrm{x}))$ transformations were applied to reinforcement rate, response rate, burst responses, and efficiency ratios, and square-root $\left(V_{\mathrm{x}}\right)$ transformations were applied to 
median and modal IRTs. All linear regressions allowed intercept and slope to vary. Within a given DRL schedule, if a set of data paths had a characteristic form other than linear, then nonlinear regression was used. One exception to this was data from the DRL 5-s schedule, which was analyzed using linear mixed-effects regression due to missing data from equipment malfunction. One of two equations was used depending on the shape of the data path across sessions. Equation 1 was a hyperbolic decay model defined as:

$$
y=\frac{a}{1+x(S)}
$$

where $a$ represents the y-intercept or the Session 1 value, $x$ represents the slope or rate of decrease across sessions, and $S$ represents successive sessions for a given DRL schedule. Equation 2 was a power function defined as:

$$
y=a\left(S^{x}\right)
$$

where the parameters $a$ and $S$ are similar to Equation 1 and $x$ represents the rate of increase across sessions. Whenever possible, both $a$ and $x$ were allowed to vary for each subject for Equations 1 and 2. When failures to fit the two-parameter model occurred, the value of $a$ was set to the mean of the Session 1 value for each group and $x$ was allowed to vary for each subject. Failures of the two-parameter model typically occurred due to between-subject variability in Session 1 values. All nonlinear analyses used raw, non-transformed data.

Transitions between each DRL schedule were analyzed using a 2 (Exposed:Control) x 2 (last:first DRL session) repeated-measures ANOVA. Specifically, the last session of each DRL schedule was compared to the first session of the subsequent DRL schedule. Session served as the within-subjects factor and exposure group served as the between-subjects factor. 


\section{Results}

\section{Nano- $\mathrm{TiO}_{2}$ aerosol exposure characterization}

Data obtained from sampling the nano- $\mathrm{TiO}_{2}$ aerosol during exposures are depicted in Figure 1. Particle agglomerates tended to be approximately in the nanometer range (Fig. 1A). Median particle diameter was estimated using four different methods. MOUDI measurements indicated a median aerodynamic diameter of $1.08 \mu \mathrm{m}$ with a geometric standard deviation (GSD) of 2.13 (Fig. 1B). ELPI measurements indicated a count median diameter of $156 \mathrm{~nm}$ with a GSD of 1.70 (Fig. 1C). SMPS and APS data were combined to yield an additional measure of count median diameter indicating a count median diameter of $184 \mathrm{~nm}$ with a GSD of 2.01 (Fig. 1D). The real-time concentration measures from an average 6-h exposure session typically approximated the target concentration of $12 \mathrm{mg} / \mathrm{m}^{3}$ (Fig. 1E). Gravimetric mass concentrations across the six, 6-h exposure sessions are shown in Fig. 1F. Across the six exposure days, four days approximated the target concentration whereas two exposure days were lower than target concentration. On the four days of target concentration exposure, rats $23 \mathrm{X}$ and $25 \mathrm{X}$ were exposed to average concentrations of $12.13 \pm 0.27 \mathrm{mg} / \mathrm{m}^{3}$ and $11.69 \pm 0.14 \mathrm{mg} / \mathrm{m}^{3}$, respectively. On the two days of unintended reduced mass concentrations, the pregnant rats were exposed to an average concentration of $4.99 \pm 0.11 \mathrm{mg} / \mathrm{m}^{3}$.

\section{Experiment 1: Response Acquisition}

All statistical outcomes for Experiment 1 can be found in Table 1.

During feeder training, each rat consumed all 60 food pellets within the session. Thirtysix hours after feeder training, the 8-h response acquisition session occurred. Overall, response acquisition was variable within groups (see Appendix B for individual cumulative records). Due to this variability, data were analyzed two ways. First, regardless of response characteristics, all 
rats were included for statistical analysis. Second, only rats that earned more than 20 pellets during the session were included (range $=4-351$ pellets across all rats). This 20 -pellet criterion was arbitrary and was chosen primarily to allow at least two rats in each group in the $2 \times 2$ ANOVA.

Figure 2 shows pellets earned, obtained delay, active lever responses, and inactive lever responses from the response acquisition session including all rats. While there was no effect of nano- $\mathrm{TiO}_{2}$ exposure for any measure (all $p$ 's $\left.>0.44\right)$, males earned fewer pellets $(F[1,27]=5.61$, $p=0.025$; Fig. 2A $)$ and had fewer active $(F[1,27]=6.04, p=0.02$; Fig. 2C $)$ and inactive responses $(F[1,27]=7.76, p<0.01$; Fig. 2A $)$. There was also a Group $\times$ Sex interaction for obtained delays $(F[1,27]=7.03, p=0.013$; Fig. 2B $)$. However, post-hoc tests were nonsignificant (all $p$ 's $>0.1$ ).

Figure 3 shows the same data as in Figure 2 but only for rats that earned at least 20 pellets during the acquisition session. For pellets earned, there was a Group $\times$ Sex interaction $(F[1,27]=5.65, p=0.035$; Fig. 3A $)$. Post-hoc tests indicated that, in this subset of rats, nano$\mathrm{TiO}_{2}$-exposed males earned fewer pellets than control males $(p=0.032)$. A Group $\times$ Sex interaction was also present for active lever responses $(F[1,27]=8.31, p=0.014$; Fig. 3C). Posthoc tests revealed that, similar to pellets earned, $\mathrm{TiO}_{2}$-exposed males had fewer active lever responses than control males $(p=0.039)$. For obtained delay, there a significant Group $\times$ Sex interaction $(F[1,27]=8.31, p=0.014$; Fig. 3B $)$, but post hoc tests were nonsignificant (all $p$ 's $>$ 0.1). Inactive lever responses were not different between groups or sexes (all $p$ 's $>0.37$ ).

\section{Experiment 2: Spatial Discrimination Reversal}

All statistical outcomes for Experiment 2 can be found in Tables 2 and 3. 
Once responding to both levers had been established, spatial discrimination training began. When a given rat completed three consecutive sessions at $\geq 85 \%$ accuracy, the correct lever changed from the left to right or right to left until four total reversals occurred across sessions. Figure 4 shows the number of sessions required to reach $85 \%$ accuracy during the original discrimination and across reversals, total errors, and response latencies across reversals. Overall, nano- $\mathrm{TiO}_{2}$-exposed males $(M=2.37, S E M=0.18)$ required more sessions to reach $85 \%$ accuracy during the original discrimination compared to control males $(M=1.71, S E M=0.17)$ $(t[13]=2.53, p=0.025$; Fig. 4G) with no difference observed in females $(t[12]=0.89, p=0.38)$. There were no group differences in sessions to criterion across reversals for either sex ( $p$ 's > 0.064; Fig. 4A/B).

On Session 1 of each reversal, errors decreased across reversals, but there was no difference between groups for male ( $p$ 's $>0.47$; Fig. 4C) or female rats ( $p$ 's $>0.068$; Fig. 4D). Response latencies were also not significantly different between groups, although, shorter latencies observed in nano- $\mathrm{TiO}_{2}$-exposed males approached significance $(p=0.059 ; \mathbf{F i g}$. 4E), which was evident not in females $(p=0.78$; Fig. 4F). For error patterning analyses, males exposed to nano- $\mathrm{TiO}_{2}$ again showed nearly significant fewer error runs during Reversal 1 ( $p=$ 0.06), but also had shallower slopes relative to control males $(\beta=0.48, t=2.45, p=0.018$; Fig. 5A) without similar effects in females ( $p$ 's $>0.09$; Fig. 5B). There were no group differences for either sex in first-run error (male: p's > 0.49; Fig. 5C; female: p's > 0.97; Fig. 5D). Unlike error runs, nano- $\mathrm{TiO}_{2}$-exposed males showed nearly significant increases in Reversal 1 error run lengths relative to control males $(\beta=1.12, t=1.95, p=0.056$; Fig. $5 \mathrm{E})$ with no difference in slope $(p=0.1)$, which was not evident in females ( $p$ 's $>0.86$; Fig. 5F). Trial-by-trial analyses also showed few differences between exposure groups for both sexes. Across reversals, Session 
1 correct:stay ratios were equivalent in males ( $p$ 's $>0.18$; Fig. 6A). Nano- $\mathrm{TiO}_{2}$-exposed females showed nearly significant increased correct:stay ratios during Reversal $1(p=0.066)$ but did show shallower slopes relative to control females $(\beta=-0.34, t=2.15, p=0.037$; Fig. 6B). No differences were detected for incorrect:shift ratios during Session 1 across reversals for either sex (p’s > 0.14; Fig. 6C/6D).

On Session 2 of each reversal, male-specific impairments were more apparent. Nano$\mathrm{TiO}_{2}$-exposed males had slightly more total errors during Reversal 1 relative to control males ( $\beta$ $=1.19, t=2.2, p=0.03$; Fig. 7A) and steeper slopes indicating that responding approached controls levels across reversals $(\beta=-0.39, t=2.17, p=0.03$; Fig. 7A). No differences were detected in females ( $p$ 's $>0.38$; Fig. 7B). Similar to trends observed during Session 1 reversals, $\mathrm{TiO}_{2}$-exposed males showed consistently shorter response latencies relative to control males $(\beta=$ $-1.73, t=3.65, p=0.001$; Fig. 7C), and $\mathrm{TiO}_{2}$-exposed male latencies remained constant across reversals whereas control male latencies decreased $(ß=0.32, t=3.3, p=0.002$; Fig. 7C). Females did not show any consistent differences in response latencies $(p=0.31)$, although $\mathrm{TiO}_{2}$ exposed females showed slightly steeper slopes than control females $(\beta=-0.24, t=2.08, p=$ 0.044; Fig. 7D). There were no group differences in error runs for males ( $p$ 's $>0.7$; Fig. 8A) or females ( $p$ 's $>0.57$; Fig. 8B). First-run errors, however, were significantly higher in nano- $\mathrm{TiO}_{2-}$ exposed males during Reversal $1(\beta=1.47, t=2.62, p=0.01$; Fig. 8C $)$ and decreased across reversals $(\beta=-0.46, t=2.31, p=0.025$; Fig. 8C). No differences in first-run errors were detected in females ( $p$ 's $>0.82$; Fig. 8D), although a transient increase was observed in $\mathrm{TiO}_{2}$-exposed females during Reversal 2. Nano- $\mathrm{TiO}_{2}$-exposed males also showed higher error run lengths during Session 2 of the first reversal as well $(\beta=1.26, t=2.11, p=0.039$; Fig. 8E $)$ and decreased across sessions $(~(\beta=-0.46, t=2.34, p=0.023$; Fig. 8E), which was likely due to 
differences in first-run error. No differences in error run lengths were detected in females ( $p$ 's $>$ 0.87; Fig. 8F). Similarly, no group differences were detected in Session 2 correct:stay ratios (male: $p$ 's $>0.08$; Fig. 9A; female: $p$ 's $>0.13$ ) or incorrect:shift ratios (male: $p$ 's $>0.063$; Fig. 9C; female: $p$ 's $>0.55$; Fig. 9D).

Because behavioral impairment was primarily driven by the first and second reversals, responding during the first three sessions of the first two reversals was also analyzed to assess within-reversal behavioral adjustment. This analysis was limited to the first three sessions to avoid reductions in statistical power due to floor effects (Morris-Schaffer et al., 2018). During Reversal 1, no group differences were detected across sessions for either sex in total error ( $p$ 's > 0.45; Fig. 10A/B), correct:stay ratios ( $p$ 's $>0.13$; Fig. 10C/D), or incorrect:shift ratios ( $p$ 's $>$ 0.11; Fig. 10E/F). Similarly, no differences were detected across sessions for either sex in error runs ( $p$ 's $>0.086$; Fig. 11A/B) or first-run error ( $p$ 's $>0.24$; Fig. 11C/D). However, nano- $\mathrm{TiO}_{2-}$ exposed males showed higher error lengths during Session 1 of Reversal 1 ( $\beta=1.08, t=2.4, p=$ 0.022; Fig. 11E), which was not evident in females $(p=0.84$; Fig. 11F). Responding across sessions during Reversal 2 also showed negligible evidence of impairment between groups. There were no group differences for either sex in Reversal 2 total error ( $p$ 's $>0.17$; Fig. 12 A/B) or incorrect:shift ratios ( $p$ 's $>0.14$; Fig. $12 \mathrm{E} / \mathbf{F}$ ). Differences between $\mathrm{TiO}_{2}$-exposed and control males for correct:stay ratios during Session 1 approached significance $(B=-1.02, t=1.96, p=$ 0.056; Fig. 12C), which was not evident in females $(p=0.99$; Fig. 12D). Again, there were no group differences detected across sessions for either sex in error runs $(p$ 's $>0.22 ; \mathbf{F i g} . \mathbf{1 3 A} / \mathbf{B})$, first-run error ( $p$ 's $>0.49$; Fig. 13C/D), or error run length ( $p$ 's $>0.4$; Fig. 13E/F). 


\section{Experiment 3: DRL}

All statistical outcomes for Experiment 3 can be found in Tables 4 (linear regressions), 5 (nonlinear regressions), 6 (DRL transition - male), and 7 (DRL transition - female)

DRL 5-s schedule. Following a 60-day break from behavioral testing, rats were trained to respond on a DRL 5-s schedule. Overall, responding readily adapted to the contingencies of the DRL 5-s schedule in both groups. Figure 14 shows reinforcement rate, response rate, and burst responses for male and female rats across the 10 sessions of DRL 5 s. Overall, reinforcement rates slightly increased (males) or remained constant (females) across sessions. Response rates did not change systematically across sessions for either sex, and burst responses sharply decreased after the first session and remained constant thereafter. There were no significant differences between groups for any dependent measure (all $p$ 's $>0.11$; Fig. 14A-F). Figure 15 shows efficiency ratios, median IRT, and modal IRT across sessions for DRL 5. Again, as expected, responding become more efficient and pauses in responding approximated the DRL 5s interval across sessions. No group differences were found for either sex in efficiency ratios or IRT measures (all p’s $>0.25$; Fig. 15A-F).

DRL 10-s schedule. After 10 sessions of the DRL 5-s schedule, a DRL 10-s schedule began the following session with no signal to subjects. Figure 16 shows reinforcement rates, response rates, and burst responses across the 15 sessions of DRL $10 \mathrm{~s}$. Recall that 150 pellets could be earned during the first five sessions, which was reduced to 60 pellets for the remaining 10 sessions. Like the DRL 5-s schedule, responding adapted to the DRL 10-s schedule in both groups, but in a more progressive manner. No differences were found between exposure groups for reinforcement rates or response rates for either sex ( $p$ 's $>0.09$; Fig. 16A-D). Burst responses decreased and became less variable following the reduction in maximum pellets at Session 6; 
thus, linear regressions were used to analyze burst responses both before and, separately, after this change. Burst responses were not different between groups at either time point (all $p$ 's $>$ 0.12; Fig. 16E/F). Figure 17 shows efficiency ratios and IRT measures across sessions during DRL 10. Efficiency ratios in both exposure groups decreased at similar rates and stabilized to similar levels after approximately five sessions ( $p=0.86$; Fig. 17A/B). Similarly, median IRTs increased across sessions with no effect of nano- $\mathrm{TiO}_{2}$ exposure (Fig. 17C/D). In contrast to median IRT, nano- $\mathrm{TiO}_{2}$-exposed males had steeper slopes in modal IRT relative to controls $(t=$ 2.33, $p=0.02$; Fig. 17E) suggesting a mild overestimation of the 10-s DRL interval, which was not evident in females $(p=0.17)$.

DRL 20-s schedule. After 15 sessions of the DRL 10-s schedule, a DRL 20-s schedule began the following session and was in effect for 15 sessions. Figure 18 shows reinforcement rates, response rates, and burst responses across sessions for the DRL 20-s schedule. Overall, reinforcement rates increased, and response rates and burst responses decreased across sessions. No group differences were detected for either sex in reinforcement rates or response rates ( $p$ 's $>$ 0.23 ; Fig. 18A-D). Nano- $\mathrm{TiO}_{2}$-exposed males, however, did show slightly shallower slopes in burst IRTs across sessions relative to control males $(B=0.02, t=2.36, p=0.034$; Fig. 18E) suggesting elevated burst responses with no such effect in females $(p=0.51 ;$ Fig. 18F). Figure 19 shows response efficiency and IRT measures across sessions during the DRL 20-s schedule. In both males and females, efficiency ratios decreased rapidly whereas median and modal IRT approached the 20-s criterion gradually. No group differences were detected for either sex in efficiency ratios ( $p$ 's $>0.26$; Fig. 19A/B) or modal IRT ( $p$ 's $>0.62$; Fig. 19E/F). Interestingly, nano- $\mathrm{TiO}_{2}$-exposed males showed slightly shallower slopes in median IRT across sessions 
relative to control males $(~(=-0.06, t=2.23, p=0.04$; Fig. 19C) without a similar effect in females ( $p=0.28$; Fig. 19D).

DRL 30-s schedule. Following the DRL 20-s schedule, a DRL 30-s schedule began the following session and was in effect for 30 sessions. To allow for similar comparison to outcomes of previous DRL schedules, the first 15 sessions of DRL 30 s were analyzed in addition to all 30 sessions. Figure 20 shows reinforcement rates, response rates, and burst responses across DRL 30 s sessions. Like the previous DRL schedules, reinforcement rates increased, and response rates and burst responses decreases across sessions. During the first 15 sessions, there was no difference in reinforcement rates between $\mathrm{TiO}_{2}$-exposed and control females. There was, however, a near-significant difference in slopes between nano- $\mathrm{TiO}_{2}$-exposed and control males for reinforcement rate $(B=-0.06, t=2.12, p=0.054$; Fig. 20A $)$ during the first 15 sessions possibly highlighting the continued increase in reinforcement rates in control males while rates for $\mathrm{TiO}_{2}$-exposed males flattened. Response rates during the first 15 sessions nearly mirrored the trends in reinforcement rate for both sexes, but group comparisons were nonsignificant (all p's > 0.1; Fig. 20C/D). Similarly, burst responses were not different between groups for either sex (all p's > 0.26; Fig. 20E/F). Across all 30 sessions, no differences were found in reinforcement rates or burst responses for either males or females (all p's >0.23; Fig. 20 A/B/E/F). Interestingly, nano- $\mathrm{TiO}_{2}$-exposed males had shallower slopes in response rate than control males across all DRL 30 s sessions $(t=2.15, p=.035$; Fig. 20C) but not females $(p=0.57$; Fig. 20D $)$. High response rates are counterproductive on a DRL schedule; thus, this suggests disrupted response inhibition in nano- $\mathrm{TiO}_{2}$-exposed males.

Figure 21 shows response efficiency and IRT measures across sessions for DRL $30 \mathrm{~s}$. In males, unreinforced:reinforced response ratios decreased and IRT measures increased steadily 
across sessions. In contrast, these measures for females changed abruptly (efficiency ratios and median IRT) during the first several sessions or approximated asymptotic performance from the onset of the DRL 30-s schedule (modal IRT). During the first 15 sessions, nano- $\mathrm{TiO}_{2}$-exposed males and females had shallower and steeper slopes, respectively, in efficiency ratios (male: $t=$ 2.61, $p=0.02$; female: $t=2.75, p=0.006$; Fig. 21A/B) indicating inefficient responding relative to control rats. It is important to note, again, that group differences observed in males were not evident from the onset of DRL $30 \mathrm{~s}$ - differences emerged as sessions progressed. For females, group differences were driven by inefficient responding in $\mathrm{TiO}_{2}$-exposed rats during the first two sessions but approximated control levels thereafter. There were no group differences in median or modal IRTs during the first 15 sessions of DRL 30 s for either sex (all p's $>0.11$; Fig. 21CF). Across all 30 sessions, there were no group differences observed in response efficiency or IRT measures. The lack of group differences in response efficiency across all sessions likely reflects detected differences during the first 15 sessions subsiding as sessions progressed.

DRL transitions. In addition to session-by-session analyses of DRL responding, transitions between the different DRL schedules were also assessed by comparing the last session of each DRL schedule to the first session of the subsequent DRL schedule. Figure 22 shows reinforcement rate, response rate, and burst responses for each DRL transition. There were no differences between groups for either sex on these measures of DRL transitions (all $p$ 's $>0.13$; Fig. 22A-F). Figure 23 shows response efficiency and IRT measures for each DRL transition. Overall, there were no differences between control and $\mathrm{TiO}_{2}$-exposed males for any of these measures (all $p$ 's $>0.15$ ). There was a significant DRL x Group interaction for modal IRT during the transition from the DRL 10 to DRL $20(\mathrm{~F}[1,13]=5.62, p=0.03$; Fig. 23E), but post hoc tests did not reveal any significant comparisons. Similarly, no differences were detected in females for 
either efficiency ratios or median IRT (all $p$ 's $>0.07$; Fig. 23B/D). There was, however, a significant DRL x Group interaction for modal IRT during the transition from DRL 5 to DRL 10 for females $(\mathrm{F}[1,12]=4.9, p=0.046$; Fig. 23F $)$. Post hoc tests revealed that modal IRT in nano$\mathrm{TiO}_{2}$-exposed females increased from the last session of DRL $5 \mathrm{~s}$ to the first session of DRL $10 \mathrm{~s}$ with no change in modal IRT detected in control females.

\section{Discussion}

\section{Overview of findings}

The aim of the present series of experiments was to evaluate behavioral impairment in adult male and female rats following gestational exposure to nano- $\mathrm{TiO}_{2}$ aerosols. Behavioral flexibility and response inhibition served as primary behavioral targets, with acquisition of an operant response in a delayed-reinforcement context serving as a novel extension of previous work. Accordingly, this is the first reported study to date evaluating effects of maternal nano$\mathrm{TiO}_{2}$ inhalation on impairment of operant behavior in adult offspring. Furthermore, the nano$\mathrm{TiO}_{2}$ exposure regimen was selected to model, insofar as possible, a more realistic exposure route and dose relative to previous work on ENM neurotoxicity.

Collectively, although no exposure-related differences were found in response acquisition (Exp. 1), male-specific deficits in nano- $\mathrm{TiO}_{2}$-exposed rats were detected in both the spatial discrimination (Exp. 2) and DRL tasks (Exp. 3), which partially mirror previous findings of developmental UFP exposures in mice (Cory-Slechta et al., 2018; Morris-Schaffer et al., 2018). These deficits also were not initially apparent but emerged following continued experience with the given tasks. For example, the first day of each discrimination reversal produced disruptions in responding to an equal extent in both exposure groups. However, on the day following each reversal, responding in nano- $\mathrm{TiO}_{2}$-exposed males continued to be disrupted via increased errors, 
which were likely driven by the first two reversals. Similarly, no systematic differences in DRL responding were evident until the final DRL 30-s schedule. Increased response rates and inefficiency ratios were primarily evident during the first 15 sessions, which approximated control levels as sessions progressed.

\section{Lack of impairment in response acquisition with delayed reinforcement}

Overall, results from the response acquisition experiment did not reveal any consistent exposure-related deficits. This lack of exposure effect was primarily due to variability in airexposed males rather than nano- $\mathrm{TiO}_{2}$-exposed males. For example, two control males earned over 190 pellets while no nano- $\mathrm{TiO}_{2}$-exposed males earned over 40 pellets. Variability was relatively consistent between female exposure groups. The 20-s unsignaled delay likely was the primary variable driving this inconsistent pattern in response acquisition. Visual inspection of cumulative records generated from the 8-h acquisition session underscores this heterogeneity of acquisition (see Appendix B). This delay was chosen to mirror a previous study that used the same procedure to highlight acquisition differences between Lewis and Fischer 344 rats (Anderson \& Elcoro, 2007), rat strains which differ in basal dopamine receptor density in brain regions critical for goal-directed behavior (e.g., the striatum and nucleus accumbens) (Flores, Wood, Barbeau, Quirion, \& Srivastava, 1998; Sánchez-Cardoso et al., 2009). In mice, gestational exposure to high-dose nano- $\mathrm{TiO}_{2}$ increased dopaminergic tone in prefrontal cortex regions including the striatum six weeks after birth (Takahashi et al., 2010), but it is unclear if the exposure regimen used in the current study perturbs dopamine in a similar manner in rats or if such perturbation would impact response acquisition in a delayed reinforcement context.

An array of methods has been used to determine environmental and biological determinants of response acquisition in a delayed reinforcement context. For example, the delay 
interval between response and reinforcement can be signaled or unsignaled, responding during the delay interval can reset the delay or have no effect upon the delay, reinforcer magnitude or food restriction can be manipulated, acquisition can be measured across multiple sessions or one extended session, or, other variables being constant, psychoactive drugs may be administered prior to the acquisition session (Doughty, Galuska, Dawson, \& Brierley, 2012; Lattal \& Williams, 2006; Lattal \& Gleeson, 1990; LeSage, Byrne, \& Poling, 1996; Wilkenfield, Nickel, Blakely, \& Poling, 1992). In general, robust establishing operations (e.g., adequate food restriction) coupled with short delays that are signaled and nonresetting tend to facilitate acquisition relative to long, unsignaled delays during which responses reset the delay (Wilkenfield et al., 1992). Thus, in selecting reinforcement parameters in the context of neurotoxicity testing, as in the current study, it is optimal to have an effective balance between the reinforcement delay being so salient and immediate that all rats acquire responding and the delay being so inconspicuous and extended that no rats acquire responding. Interestingly, a parametric analysis of nonresetting delays of $0,4,8$, and $16 \mathrm{~s}$ during an 8-h response acquisition similar to that used here showed a positive relation between reinforcement delay and acquisition suggesting that longer delays do not necessarily preclude consistent acquisition between subjects (Wilkenfield et al., 1992). Nonetheless, the current study suggests that a 20-s nonresetting delay may lead to inconsistent response acquisition to the extent that conclusions regarding toxicant exposure, or even drug administration, are tentative at best. Future studies using this procedure should consider the interaction of the reinforcement parameters mentioned above prior to investigating this relatively understudied aspect of operant behavior. 


\section{Impairment in reversal learning}

In the current study, nano- $\mathrm{TiO}_{2}$-exposed males, but not females, displayed deficits in discrimination reversal learning. In addition to requiring more sessions to reach the mastery criterion during original discrimination training, nano- $\mathrm{TiO}_{2}$-exposed males had increased error, shorter response latencies, and increased first-run error during Session 2 of the first reversal with these effects diminishing across reversals. It is interesting to note that no differences were found in these measures during Session 1 of each reversal, or the day the contingencies reversed, but emerged the following day. Most studies using discrimination reversals to assess neurotoxicity primarily plot performance across reversals only for the session in which contingencies reversed (i.e., Session 1; Paletz et al., 2007; Reed et al., 2006; Widholm, Seo, Strupp, Seegal, \& Schantz, 2003). Analyses of Session 2 were included in the present study to determine if gestational nano$\mathrm{TiO}_{2}$ exposure may affect the extent to which rats "remember" the change in reinforcement contingencies. In this regard, Session 1 may be interpreted as a measure of behavioral flexibility whereas Session 2 could be considered as a stimulus control or "memory" test. Importantly, firstrun errors, but not total error runs or correct:stay ratios, were elevated in nano- $\mathrm{TiO}_{2}$-exposed males indicating that responding perseverated on the incorrect lever at the beginning of the session, then switched to the correct lever as the session progressed. This finding suggests that the prior experience of the reversal (i.e., Session 1) in nano- $\mathrm{TiO}_{2}$-exposed males was not sufficient to maintain that discrimination the following session. This effect was also not likely due to any motivational disruption as response latencies were much shorter in nano- $\mathrm{TiO}_{2-}$ exposed male rats relative to control male rats.

The observed effects of nano- $\mathrm{TiO}_{2}$ exposure on reversal learning are interesting for at least two reasons. First, despite detecting greater total errors and first-run error during Session 2 
of the first reversal, it could be argued that the rats in the current study had more experience pressing both response levers prior to discrimination reversals relative to previous studies using similar procedures. This is largely due to 1) the response acquisition session and 2) the alternating-FR training. In the majority of multi-experiment evaluations of behavioral impairment, discrimination learning is the first experiment conducted because of its sensitivity to reinforcement history (Paletz et al., 2007; Reed et al., 2006; Rice, 1998). The current study, however, required naïve rats for the initial acquisition experiment so some experience pressing levers prior to discrimination training was unavoidable. Also, in other studies, shaping of lever pressing to the spatially distinct levers also tends to occur during separate training sessions. For example, 100 presses on the left lever one day and 100 presses on the right lever the next day (Rice, 1998). If responses to different spatial locations are required within the same session prior to the discrimination training proper, then it typically involves trial-initiation chains such as pressing a rear panel lever to produce the left or right front lever (Reed et al., 2006). To eliminate any potential lever bias from the acquisition experiment, rats in the current study received three consecutive sessions of alternating-FR sessions in which responses were required to alternate between the right and left levers every five reinforcers. It is plausible that this training increased the likelihood of switching to the alternative lever following periods of extinction during reversal sessions or otherwise masked an even greater exposure effect in nano- $\mathrm{TiO}_{2}$-exposed rats. Although, reversal learning deficits following gestational exposure to other environmental contaminants have been reported after similar alternating-FR training protocols (Widholm et al., 2003). One possible challenge to this interpretation is that, in the current study, Session 1 discrimination errors during the first reversal occurred on approximately $75 \%$ of trials for both 
groups suggesting equally high insensitivity to reversed reinforcement contingencies during that session.

Second, these data align with previous studies showing that gestational toxicant exposure may preferentially affect reversal learning (i.e., intradimensional shifts). Similar to behavioral outcomes of UFP-exposed mice reported by Morris-Schaffer and colleagues (2018), nano- $\mathrm{TiO}_{2}$ exposed male rats, and not female rats, in the current study showed impaired learning of the initial discrimination prior to reversals via increased sessions to criterion. Discrimination reversals, however, did not reveal differences between postnatal UFP-exposed and control mice, which were evident in rats following gestational nano- $\mathrm{TiO}_{2}$ exposure in the current study. Morris-Schaffer et al. (2018) reported deficits in UFP-exposed male mice only following extradimensional shifts, which require an attentional shift to another stimulus modality. That is, neonatal UFP aerosol exposures preferentially affected extradimensional shifts whereas, in the current study, gestational nano- $\mathrm{TiO}_{2}$ aerosol exposures affected discrimination reversals. While the present study did not assess extradimensional shifts, similar patterns of results have been observed following gestational vs. adolescent exposure to methylmercury $(\mathrm{MeHg})$ in which postnatal exposure affects extradimensional shifts and gestational exposure affects reversal learning or intradimensional shifts (Boomhower \& Newland, 2017; Paletz et al., 2007). Separate areas of the prefrontal cortex (PFC) control behavioral adjustment to intradimensional and extradimensional shifts, respectively. Specifically, lesions to the orbitofrontal PFC disrupt reversal learning whereas lesions to the medial PFC disrupt extradimensional shifts, which has been shown in both monkeys (Dias et al., 1996) and rats (McAlonan \& Brown, 2003). Given that extradimensional shifts were not assessed in the current study, it is unclear if the deficits detected 
in reversal learning would carry over to or be independent of impaired extradimensional shift learning.

\section{Response inhibition impairment}

Male-specific deficits in response inhibition observed in rats in the current study also align with previous data on male mice exposed to UFP during the neonatal window (CorySlechta et al., 2018). In mice, increased response rates and decreased response efficiency observed following UFP exposure were primarily present upon transitioning from a short DRL schedule to a longer DRL schedule (Cory-Slechta et al., 2018). This “transition" effect has also been observed in rats exposed during gestation to $\mathrm{MeHg}$ after transitioning from a FR 5 schedule to a DRL 10-s schedule (Paletz et al., 2006). Similar response characteristics were disrupted in nano- $\mathrm{TiO}_{2}$-exposed males in the current study, but, importantly, these effects were observed as sessions progressed rather than at DRL transition points. A similar study found that extended perinatal exposure to UFP in rats decreased total reinforcers earned on a DRL 5-, but not a DRL 10- or 20-s schedule; although, this effect was due to decreased responding overall and not response efficiency, per se (Woodward et al., 2018). In the current study, increased response rates and decreased response efficiencies observed on the DRL 30-s schedule suggest an insensitivity to the increasingly difficult DRL interval as opposed to motivational or motoric deficits. Furthermore, the latent emergence of these deficits also partially mirrors the primary findings of Experiment 2 in which reversal learning deficits were detected primarily during Session 2 of each reversal rather than the session in which contingencies reversed.

The impairments detected on the DRL 30-s schedule suggest a deficit in "impulse control" or an inability to withhold responding generally rather than interval timing, specifically. That is, higher response rates and response inefficiency coupled with a lack of differences in 
modal IRT may highlight an overall propensity to respond early rather than inaccurate or imprecise discrimination of the DRL interval. In addition to median IRT, modal IRT - in this case, defined as the most frequently occurring class of IRTs in the rightward peak of IRTs from IRT histograms - was included to provide a more direct measure of interval timing. On DRL schedules, it is common to observe a bimodal IRT distribution in which a high number of "within-bout" IRTs are observed in IRT bins shorter than 2-3 seconds, then IRT frequency drops sharply and begins to rise again as "between-bout" IRTs start approximating the criterion DRL interval (Eckard \& Kyonka, 2018; Richards, Sabol, \& Seiden, 1993). The rightward mode of IRTs is then typically considered a measure of timing accuracy to the extent that it aligns with the DRL criterion, and timing precision is represented by the width of the IRT peak around the criterion time (Richards et al., 1993). In the present study, neither nano- $\mathrm{TiO}_{2}$-exposed males nor females deviated from air-exposed controls with respect to modal IRT. From the response efficiency and median IRT data, it is plausible that nano- $\mathrm{TiO}_{2}$-exposed males may have had wider IRT distributions relative to control males; however, quantification of this IRT distribution peak width on a DRL schedule is less standardized than precision-related measures from other timing tasks (e.g., peak-interval procedure; Catania, 1970; Eckard \& Kyonka, 2018). A more formal method for dissociating response inhibition from interval timing processes generally may involve using tasks specific to one or the other process. For example, the 5-choice serial reaction time task has been used extensively to evaluate response inhibition or "impulse control" and attentional processes specifically (Pattij, Janssen, Vanderschuren, Schoffelmeer, \& van Gaalen, 2007; Robbins, 2002). Similarly, one might use a peak-interval (Eckard \& Kyonka, 2018; Catania, 1970) or temporal bisection task (Cheng, Etchegaray, \& Meck, 2007; Church \& Deluty, 1977) to probe prospective or retrospective timing processes, respectively, more effectively 
relative to a DRL schedule. In this regard, the DRL schedule could be viewed as a useful tool for identifying the presence or absence of neurobehavioral disruption with more refined tasks determining the specific behavioral mechanism(s) involved in the disruption. Overall, the current data warrant further investigation into the dissociation between impulsivity and timing-related deficits following gestational nano- $\mathrm{TiO}_{2}$ exposure.

\section{Possible neural mechanisms}

The behavioral impairment detected in reversal learning and DRL-schedule performance in the current study reflects mounting evidence of disrupted neurological development following perinatal exposure to nano- $\mathrm{TiO}_{2}$. For example, nano- $\mathrm{TiO}_{2}(100 \mathrm{mg} / \mathrm{kg}$, po $)$ exposure throughout gestation decreased cell proliferation in the hippocampus of rat offspring, which aligned with spatial memory deficits in a Morris water maze task (Mohammadipour et al., 2014). In fact, hippocampal dysfunction (e.g., decreased cell proliferation, inhibited dendrite outgrowth, oxidative stress, loss of NMDA receptors) is the most frequently reported neurological outcome following gestational nano- $\mathrm{TiO}_{2}$ exposure in rats and mice (Cui et al., 2014; Gao et al., 2011; Hong et al., 2018; Mohammadipour et al., 2014; Zhou, Ji, Chen, \& Hong, 2019; Zhou, Ji, Hong, Zhuang, \& Wang, 2019) - effects which are associated with spatial memory deficits or increased anhedonia in some instances (Cui et al., 2014; Mohammadipour et al., 2014). Similar disruptions in overall hippocampus morphology are also present following extended perinatal exposure to concentrated air pollution (Fonken et al., 2011). It is important to note, however, that most of these studies assessed neurological outcomes as early as PND 2 up to PND 70, the latter of which partially aligns with reversal learning in the current experiment (PND 80-100), but not the various DRL schedules (>PND 180). 
It is hypothesized that nano- $\mathrm{TiO}_{2}$ induces neurotoxic effects through oxidative stress mechanisms (Cui et al., 2014; Long et al., 2007) possibly initiated by increases in extracellular glutamate (Hong et al., 2015). Interestingly, postnatal UFP exposure in mice also produces increases in glutamate levels in the striatum and the hippocampus of male mice, which is associated with increased microglial inflammatory markers in the cortex (Allen, Liu, Weston, Conrad, et al., 2014; Allen, Liu, Weston, Prince, et al., 2014). The hippocampus is particularly vulnerable to glutamate excitotoxicity due to its high density of glutamate receptors (Mehta, Prabhakar, Kumar, Deshmukh, \& Sharma, 2013; Shinohara, 2012). Increased glutamate signaling produces prolonged elevations in intracellular $\mathrm{Ca}^{2+}$, which in turn activates apoptotic signaling cascades eventually leading to inflammation and cell death (Randall \& Thayer, 2018; Sattler \& Tymianski, 2000). Indeed, increases in intracellular $\mathrm{Ca}^{2+}$ and oxidative stress markers in cultured rat hippocampal neurons have been observed following incubation with nano- $\mathrm{TiO}_{2}$ suspensions (Hong et al., 2015). Similarly, exposure to UFP aerosols increases proinflammatory cytokines (i.e., IL-1B, IL-6) up to PND 55 in the brains of male mice, which is preceded by increased hippocampal glutamate concentrations at PND 14 (Allen, Liu, Pelkowski, et al., 2014). Thus, there appears to be a relation between ultrafine or nano-particulate exposure and hippocampal excitotoxicity via glutamate overproduction, which may underlie behavioral impairment following neonatal or gestational exposure to nano- $\mathrm{TiO}_{2}$ and other ultrafine aerosols.

Damage to hippocampal circuits can manifest in behavioral measures similar to those used in the current study, specifically DRL-schedule performance and discrimination learning generally. In rats and mice, hippocampal lesions routinely disrupt DRL schedule performance (Bannerman et al., 1999; Cho \& Jeantet, 2010; Deacon, Reisel, Perry, Nicholas, \& Rawlins, 2005; Sinden, Rawlins, Gray, \& Jarrard, 1986). Furthermore, these lesions tend to reduce 
response efficiency by increasing sub-criterion IRTs without increasing burst IRTs, per se (Cho \& Jeantet, 2010; Sinden et al., 1986). Importantly, decreased response efficiency on DRL schedules is present whether the lesion is localized to the ventral, dorsal, or both aspects of the hippocampus (Bannerman et al., 1999). Although, it is important to highlight that DRL schedule performance is also highly controlled by PFC and subcortical motor circuits (Cho \& Jeantet, 2010) and not exclusively by hippocampal circuits. Reflective of previous studies assessing hippocampal damage following gestational nano- $\mathrm{TiO}_{2}$ and other ultrafine aerosol exposures, the deficits in DRL performance in the current study could be a result of disrupted hippocampal function.

The extent to which hippocampal damage could be responsible for the reversal learning deficits observed in the current study is unclear. Recall that intradimensional (i.e., reversal learning) and extradimensional shifts are primarily mediated by the orbital PFC and medial PFC, respectively (Dias et al., 1996; McAlonan \& Brown, 2003). In rats, the ventral hippocampus sends efferent projections to the medial PFC with little-to-no innervation of the orbital PFC (Jay \& Witter, 1991; Verwer, Meijer, Van Uum, \& Witter, 1997). There is corroborating evidence that cytotoxic lesions of the ventral hippocampus in neonate rats (i.e., PND 6) leads to disrupted formation of the medial PFC and increased errors in an extradimensional, but not intradimensional, shift task (Marquis, Goulet, \& Doré, 2008; Placek, Dippel, Jones, \& Brady, 2013). Similarly, exposure to UFP in neonate mice (PND 7-10) increased errors on an extradimensional, but not intradimensional, shift task in males (Morris-Schaffer et al., 2018) and increased glutamate concentrations in the hippocampus of male mice (Allen, Liu, Pelkowski, et al., 2014). Given these considerations, it appears that hippocampal damage produced by UFP or nano- $\mathrm{TiO}_{2}$ exposure should preferentially disrupt extradimensional shifts and not 
intradimensional shifts. Again, because extradimensional shifts were not evaluated in the current study, it unclear if these deficits would have been detected. Future investigations of effects of gestational nano- $\mathrm{TiO}_{2}$ exposure on behavioral flexibility should consider study design strategies to implement both types of dimensional shift paradigms.

\section{Sex as a determinant of neurotoxicity}

In line with previous research, behavioral impairment was primarily detected in nano$\mathrm{TiO}_{2}$-exposed males rather than females (Cory-Slechta et al., 2018). Although precise mechanisms remain unclear, several hypotheses have been proposed to account for male-biased susceptibility to developmental neurotoxicant exposure that may underlie male-biased neurodevelopmental disorders including autism spectrum disorder (ASD) and ADHD (Kern et al., 2017). At least two important mechanisms include sex differences in antioxidant systems and steroid hormones that promote neuroprotection (Villa, Vegeto, Poletti, \& Maggi, 2016). A chief function of antioxidants is to protect against oxidative damage from overproduction of reactive oxygen species (ROS) by mitochondria (Murphy, 2008; Palmer \& Paulson, 1997), which is thought to be one of the primary mechanisms of nano- $\mathrm{TiO}_{2}$ neurotoxicity (Skocaj, Filipic, Petkovic, \& Novak, 2011). Although studies assessing sex differences in oxidative stress responses following nano- $\mathrm{TiO}_{2}$ exposure have yet to be conducted, several lines of evidence suggest male-biased susceptibility to ROS. In humans, various antioxidants, like glutathione, are higher in infant females than in infant males, and female fetuses tend to show more robust increases in glutathione concentrations during gestation compared to males (Lavoie \& Chessex, 1997; Rush \& Sandiford, 2003). Not only does this relation generally hold for antioxidant expression in mice, but it also correlates with sensitivity to gestational neurotoxicant (e.g., $\mathrm{MeHg}$ ) exposure such that males show a blunted antioxidant response thereby increasing toxicity 
in male mice compared to female mice (Kimiko, Akira, \& Masayasu, 1987; Ruszkiewicz, Bowman, Farina, Rocha, \& Aschner, 2016). Gestational exposure to the ROS-stimulating glucocorticoid corticosterone also shows sex-specific effects. For example, female mice exposed to corticosterone for a short time (i.e., two days) during gestation show increased antioxidant responses relative to male mice suggesting females may have enhanced oxidative stress defense mechanisms (Bartho, Holland, Moritz, Perkins, \& Cuffe, 2019). This effect is also mirrored in humans in which synthetic glucocorticoid treatment increases pro-oxidative markers and decreases anti-oxidative markers in male placental tissue as compared to female placental tissue (Stark, Hodyl, Wright, \& Clifton, 2011). Given male susceptibility to ROS and ROS production being a primary determinant of nano- $\mathrm{TiO}_{2}$ toxicity either from the gestational environment or within the developing fetus, it is possible this male-biased ROS susceptibility could underlie behavioral impairment observed here and elsewhere.

In addition to sex differences in antioxidant defense, there is evidence that estrogens, a class of steroid hormone, can mitigate negative effects of neuroinflammation (Villa et al., 2016). Estrogens (e.g., 17 $\beta$-estradiol, estrone, and estriol), typically present in higher concentrations in females relative to males, are synthesized through a series of enzymatic reactions which ultimately converts androgens into estrogens through a process called aromatization (Nelson, 2011). Estrogens, particularly $17 \beta$-estradiol, are also potent modulators of oxidative stress (Behl, Widmann, Trapp, \& Holsboer, 1995; Keaney et al., 1994). In fact, 17ß-estradiol is extremely effective at blocking glutamate-induced excitotoxicity via increases in intracellular $\mathrm{Ca}^{2+}$ (Goodman, Bruce, Cheng, \& Mattson, 1996), which, again, is thought to be an important component of nano- $\mathrm{TiO}_{2}$ neurotoxicity (Hong et al., 2015). In vivo models of brain injury also support estrogen-mediated neuroprotection. For example, in ovariectomized female rats, both 
peripheral and central administration of $17 \beta$-estradiol drastically decreased mortality following occlusion of the middle cerebral artery (Simpkins et al., 1997). Similar attenuations of brain injury have been found using glutamate-induced lesions in rats following pretreatment with $17 \beta$ estradiol (Mendelowitsch, Ritz, Ros, Langemann, \& Gratzl, 2001). Therefore, it follows that females may be protected from various sources of neurotoxicity through enhanced antioxidant response and presence of higher concentrations of neuroprotective estrogens. These mechanisms may partially explain the sex differences observed in the current study.

\section{Limitations}

Considering the findings of the current study, at least two limitations warrant attention. First, control and nano- $\mathrm{TiO}_{2}$-exposed rats were represented by only two litters, respectively. It is typical for studies using gestational exposure models to include one or two subjects from multiple litters in each group such that litter can be treated as a covariate in statistical analyses (Cory-Slechta et al., 2018; Morris-Schaffer et al., 2018; Reed et al., 2006). Thus, it is possible that the behavioral impairment observed in the current study could simply be due to differences across litters and not nano- $\mathrm{TiO}_{2}$ exposure, per se. Two points may be made against this interpretation. First, impairment was detected in the same group of male rats, which came from separate litters across two separate experiments at distinctly different developmental time points. For example, spatial discrimination deficits were detected between PND 80-100 and deficits in DRL responding were detected over six months of age. Second, $\mathrm{TiO}_{2}$-exposed male and female rats in the current study came from the same litters, and the sex-dependent effects observed here partially mirror results of previous studies assessing behavioral deficits following UFP exposures in mice sampled across 12 litters (Cory-Slechta et al., 2018; Morris-Schaffer et al., 2018). 
A second limitation is that only one nanomaterial at a single aerosol concentration was used in the current study. Nano- $\mathrm{TiO}_{2}$ was selected primarily because of its ubiquity in industrial applications and history as a prototypical nanomaterial. However, other nanomaterial aerosols have been characterized in the context of developmental neurotoxicity and understanding how different nanomaterials affect cognitive development is a crucial step in hazard identification. For example, neonatal exposure to elemental carbon, or carbon black, aerosols $\left(45 \mathrm{ug} / \mathrm{m}^{3}\right) \mathrm{did}$ not produce any detectable behavioral impairment on a fixed-interval or DRL schedule of reinforcement in mice (Morris-Schaffer, Merrill, Jew, et al., 2019). Additionally, a similar exposure regimen to diesel exhaust aerosols $\left(100 \mu \mathrm{g} / \mathrm{m}^{3}\right)$ produced little-to-no behavioral deficits in mice (Morris-Schaffer, Merrill, Wong, et al., 2019). To address this limitation, an interesting future direction is to evaluate effects of co-exposure of different types of nanomaterials or nanomaterials in conjunction with other, more common environmental pollutants to model realworld exposures more effectively.

\section{Conclusion}

The primary conclusion of the present study is that low-dose nano- $\mathrm{TiO}_{2}$ inhalation during pregnancy produces detectable impairment of operant behavior in adult offspring. Furthermore, these effects were primarily present in male rats, not females. Behavioral impairment was largely characterized by reduced latencies, increased errors, first-run errors, and sessions to criterion to learn an original discrimination in the spatial discrimination reversal task and increased response rates and decreased response efficiency on a DRL 30-s schedule. This extends previous work showing a similar exposure regimen to that used here increased latency to find a submerged platform in male rats (Engler-Chiurazzi et al., 2016). The current study also recapitulates 
behavioral impairment following gestational exposure to other ultrafine aerosols linked to air pollution (Cory-Slechta et al., 2018; Morris-Schaffer et al., 2018).

As nanomaterials continue to be increasingly produced for industrial, medical, and consumer purposes, identifying the harmful consequences of unintended exposures is extremely important (Hendren et al., 2011; Hougaard et al., 2015). The extent to which engineered nanomaterials affect development and function of the central nervous system is particularly concerning, and is becoming a more commonly investigated endpoint (Bencsik, Lestaevel, \& Guseva Canu, 2017). In this regard, operant behavioral techniques may be uniquely poised to contribute to the effort of identifying neurobehavioral consequences of ENM exposure whether the exposure is direct or developmental. Operant techniques have been routinely used in other fields of neurotoxicology, and have a long history of utility in characterizing executive function deficits and motor impairment, especially at low-level exposures (Cory-Slechta, 2010; Laties, 1978; Newland, 2010). Perhaps the current study will serve as an impetus to continue uncovering the extent to which ENM exposure induces neurobehavioral impairment. 


\section{References}

Abukabda, A. B., Bowdridge, E. C., Mcbride, C. R., Batchelor, T. P., Goldsmith, W. T., Garner, K. L., ... Nurkiewicz, T. R. (2019). Maternal titanium dioxide nanomaterial inhalation exposure compromises placental hemodynamics. Toxicology and Applied Pharmacology, 367(January), 51-61. https://doi.org/10.1016/j.taap.2019.01.024

Allen, J., Klocke, C., Morris-Schaffer, K., Conrad, K., Sobolewski, M., \& Cory-Slechta, D. A. (2017). Cognitive effects of air pollution exposures and potential mechanistic underpinnings. Current Environmental Health Reports, 4, 180-191. https://doi.org/10.1007/s40572-017-0134-3

Allen, J. L., Oberdorster, G., Morris-Schaffer, K., Wong, C., Klocke, C., Sobolewski, M., ... Cory-Slechta, D. A. (2017). Developmental neurotoxicity of inhaled ambient ultrafine particle air pollution: Parallels with neuropathological and behavioral features of autism and other neurodevelopmental disorders. NeuroToxicology, 59, 140-154. https://doi.org/10.1016/j.neuro.2015.12.014

Allen, Joshua L., Liu, X., Pelkowski, S., Palmer, B., Conrad, K., Oberdörster, G., ... CorySlechta, D. A. (2014). Early postnatal exposure to ultrafine particulate matter air pollution: Persistent ventriculomegaly, neurochemical disruption, and glial activation preferentially in male mice. Environmental Health Perspectives, 122, 939-945. https://doi.org/10.1289/ehp.1307984

Allen, Joshua L., Liu, X., Weston, D., Conrad, K., Oberdörster, G., \& Cory-Slechta, D. A. (2014). Consequences of developmental exposure to concentrated ambient ultrafine particle air pollution combined with the adult paraquat and maneb model of the Parkinson's disease 
phenotype in male mice. NeuroToxicology, 41, 80-88.

https://doi.org/10.1016/j.neuro.2014.01.004

Allen, Joshua L., Liu, X., Weston, D., Prince, L., Oberdörster, G., Finkelstein, J. N., ... CorySlechta, D. A. (2014). Developmental exposure to concentrated ambient ultrafine particulate matter air pollution in mice results in persistent and sex-dependent behavioral neurotoxicity and glial activation. Toxicological Sciences, 140(1), 160-178. https://doi.org/10.1093/toxsci/kfu059

Allen, Joshua L., Oberdörster, G., Morris-Schaffer, K., Wong, C., Klocke, C., Sobolewski, M., ... Cory-Slechta, D. A. (2017). Developmental neurotoxicity of inhaled ambient ultrafine particle air pollution: Parallels with neuropathological and behavioral featues of autism and other neurodevelopmental disorders. Neurotoxicology, 59, 140-154. https://doi.org/10.1016/j.neuro.2015.12.014.Developmental

Allen, Joshua L, Conrad, K., Oberdoerster, G., Johnston, C. J., Sleezer, B., \& Cory-Slechta, D. A. (2013). Developmental Exposure to Concentrated Ambient Particles and Preference for Immediate Reward in Mice. Environmental Health Perspectives, 007026(1), 32-38.

Anderson, K. G., \& Elcoro, M. (2007). Response acquisition with delayed reinforcement in Lewis and Fischer 344 rats. Behavioural Processes, 74(3), 311-318. https://doi.org/10.1016/j.beproc.2006.11.006

Bannerman, D. M., Good, M. A., Yee, B. K., Heupel, M. J., Iversen, S. D., \& Rawlins, J. N. P. (1999). Double dissociation of function within the hippocampus: A comparison of dorsal, ventral, and complete hippocampal cytotoxic lesions. Behavioral Neuroscience, 113(6), 1170-1188. https://doi.org/10.1037/0735-7044.113.6.1170 
Bartho, L. A., Holland, O. J., Moritz, K. M., Perkins, A. V., \& Cuffe, J. S. M. (2019). Maternal corticosterone in the mouse alters oxidative stress markers, antioxidant function and mitochondrial content in placentas of female fetuses. Journal of Physiology, 597(12), 30533067. https://doi.org/10.1113/JP277815

Behl, C., Widmann, M., Trapp, T., \& Holsboer, F. (1995). 17-B Estradiol Protects Neurons From Oxidative Stress-Induced Cell Death in Vitro. Biochemical and Biophysical Research Communications, Vol. 216, pp. 473-482. https://doi.org/10.1006/bbrc.1995.2647

Bencsik, A., Lestaevel, P., \& Guseva Canu, I. (2017). Nano- and neurotoxicology: An emerging discipline. Progress in Neurobiology. https://doi.org/10.1016/j.pneurobio.2017.10.003

Bencsik, A., Lestaevel, P., \& Guseva Canu, I. (2018). Nano- and neurotoxicology: An emerging discipline. Progress in Neurobiology, 160, 45-63. https://doi.org/10.1016/j.pneurobio.2017.10.003

Boisgontier, M. P., \& Cheval, B. (2016). The ANOVA to mixed model transition. Neuroscience and Biobehavioral Reviews, 68, 1004-1005. https://doi.org/10.1016/j.neubiorev.2016.05.034

Boomhower, S. R., \& Newland, M. C. (2017). Effects of adolescent exposure to methylmercury and d-amphetamine on reversal learning and an extradimensional shift in male mice. Experimental and Clinical Psychopharmacology, 25, 64-73. https://doi.org/10.1037/pha0000107

Box, G. E. P., \& Cox, D. R. (1964). An analysis of transformations. Journal of the Royal Statistical Society. Series B, 26(2), 211-252. 
Bushnell, P. J. (1988). Behavioral effects of acute p-xylene inhalation in rats: Autoshaping, motor activity, and reversal learning. Neurotoxicology and Teratology, 10, 569-577. https://doi.org/10.1016/0892-0362(88)90094-3

Catania, A. C. (1970). Reinforcement schedules and psychophysical judgments: A study of some temporal properties of behavior. In W. N. Schoenfeld (Ed.), The Theory of Reinforcement Schedules. New York: Appleton-Century-Crofts.

Cheng, R., Etchegaray, M., \& Meck, W. H. (2007). Impairments in timing, temporal memory, and reversal learning linked to neurotoxic regimens of methamphetamine intoxication. Brain Research, 1186, 255-266. https://doi.org/10.1016/j.brainres.2007.10.002

Chiu, Y. M., Hsu, H. L., Coull, B. A., Bellinger, D. C., Kloog, I., Schwartz, J., .. Wright, R. J. (2017). Prenatal particulate air pollution and neurodevelopment in urban children: Examining sensitive windows and sex-specific associations. Environment International, 87, 56-65. https://doi.org/10.1016/j.envint.2015.11.010.Prenatal

Cho, Y. H., \& Jeantet, Y. (2010). Differential involvement of prefrontal cortex, striatum, and hippocampus in DRL performance in mice. Neurobiology of Learning and Memory, 93(1), 85-91. https://doi.org/10.1016/j.nlm.2009.08.007

Church, R. M., \& Deluty, M. Z. (1977). Bisection of temporal intervals. Journal of Experimental Psychology: Animal Behavior Processes, 3(3), 216-228.

Cory-Slechta, D. A. (2010). Intermittent schedules of reinforcement as toxicological end points. In C. A. McQueen (Ed.), Comprehensive Toxicology (2nd ed., pp. 363-378). Elsevier Science. 
Cory-Slechta, D., Allen, J. L., Conrad, K., Marvin, E., \& Sobolewski, M. (2018). Developmental exposure to low level ambient ultrafine particle air pollution and cognitive dysfunction. Neurotoxicology, 69, 217-231. https://doi.org/10.1016/j.neuro.2017.12.003

Cui, Y., Chen, X., Zhou, Z., Lei, Y., Ma, M., Cao, R., ... Che, Y. (2014). Prenatal exposure to nanoparticulate titanium dioxide enhances depressive-like behaviors in adult rats. Chemosphere, 96, 99-104. https://doi.org/10.1016/j.chemosphere.2013.07.051

Cupaioli, F. A., Zucca, F. A., Boraschi, D., \& Zecca, L. (2014). Engineered nanoparticles. How brain friendly is this new guest? Progress in Neurobiology, 119-120, 20-38. https://doi.org/10.1016/j.pneurobio.2014.05.002

Deacon, R. M. J., Reisel, D., Perry, V. H., Nicholas, J., \& Rawlins, P. (2005). Hippocampal scrapie infection impairs operant DRL performance in mice. Behavioural Brain Research, 157(1), 99-105. https://doi.org/10.1016/j.bbr.2004.06.013

Di, Q., Dai, L., Wang, Y., Zanobetti, A., Choirat, C., Schwartz, J. D., \& Dominici, F. (2017). Association of short-term exposure to air pollution with mortality in older adults. Journal of the American Medical Association, 318, 2446-2456. https://doi.org/10.1001/jama.2017.17923

Di, Q., Wang, Y., Zanobetti, A., Wang, Y., Koutrakis, P., Choirat, C., ... Schwartz, J. D. (2017). Air pollution and mortality in the medicare popoulation. New England Journal of Medicine, 376, 2513-2522. https://doi.org/10.1056/NEJMoa1702747

Dias, R., Robbins, T. W., \& Roberts, A. C. (1996). Dissociation in prefrontal cortex of affective and attentional shifts. Nature, Vol. 380, pp. 69-72. https://doi.org/10.1038/380069a0 
Doughty, A. H., Galuska, C. M., Dawson, A. E., \& Brierley, K. P. (2012). Effects of reinforcer magnitude on response acquisition with unsignaled delayed reinforcement. Behavioural Processes, 90(2), 287-290. https://doi.org/10.1016/j.beproc.2012.02.016

Eckard, M. L., \& Kyonka, E. G. E. (2018). Differential reinforcement of low rates differentially decreased timing. Behavioural Processes, 151(March), 111-118. https://doi.org/10.1016/j.beproc.2018.02.022

Elder, A., Gelein, R., Silva, V., Feikert, T., Opanashuk, L., Carter, J., ... Oberdörster, G. (2006). Translocation of inhaled ultrafine manganese oxide particles to the central nervous system. Environmental Health Perspectives, 114(8), 1172-1178. https://doi.org/10.1289/ehp.9030

Engler-Chiurazzi, E. B., Stapleton, P. A., Stalnaker, J. J., Ren, X., Hu, H., Nurkiewicz, T. R., ... Simpkins, J. W. (2016). Impacts of prenatal nanomaterial exposure on male adult SpragueDawley rat behavior and cognition. Journal of Toxicology and Environmental Health - Part A: Current Issues, 79(11), 447-452. https://doi.org/10.1080/15287394.2016.1164101

Feynman, R. P. (1960). There's plenty of room at the bottom. Engineering and Science, Vol. 23, pp. 22-36. https://doi.org/10.1016/j.applthermaleng.2013.04.038

Flores, G., Wood, G. K., Barbeau, D., Quirion, R., \& Srivastava, L. K. (1998). Lewis and Fischer rats: A comparison of dopamine transporter and receptors levels. Brain Research, 814(1-2), 34-40. https://doi.org/10.1016/S0006-8993(98)01011-7

Fonken, L. K., Xu, X., Weil, Z. M., Chen, G., Sun, Q., Rajagopalan, S., \& Nelson, R. J. (2011). Air pollution impairs cognition, provokes depressive-like behaviors and alters hippocampal cytokine expression and morphology. Molecular Psychiatry, 16(10), 987-995. https://doi.org/10.1038/mp.2011.76 
Fox, A. E. (2018). The future is upon us. Behavior Analysis: Research and Practice, 18, 144150. https://doi.org/http://dx.doi.org/10.1037/bar0000106

Fu, J., Gao, J., Gong, L., Ma, Y., Xu, H., Gu, Z., ... Fan, X. (2018). Silica nanoparticle exposure during the neonatal period impairs hippocampal precursor proliferation and social behavior later in life. International Journal of Nanomedicine, 13, 3593-3608.

Gao, X., Yin, S., Tang, M., Chen, J., Yang, Z., Zhang, W., ... Wang, M. (2011). Effects of developmental exposure to $\mathrm{TiO} 2$ nanoparticles on synaptic plasticity in hippocampal dentate gyrus area: An in vivo study in anesthetized rats. Biological Trace Element Research, 143(3), 1616-1628. https://doi.org/10.1007/s12011-011-8990-4

Gaté, L., Disdier, C., Cosnier, F., Gagnaire, F., Devoy, J., Saba, W., ... Mabondzo, A. (2017). Biopersistence and translocation to extrapulmonary organs of titanium dioxide nanoparticles after subacute inhalation exposure to aerosol in adult and elderly rats. Toxicology Letters, 265, 61-69. https://doi.org/10.1016/j.toxlet.2016.11.009

Geiser, M, \& Kreyling, W. G. (2010). Deposition and biokenetics of inhaled nanoparticles. Particle and Fibre Toxicology, 7(2), 2-19.

Geiser, Marianne, Casaulta, M., Kupferschmid, B., Schulz, H., Semmler-Behnke, M., \& Kreyling, W. (2008). The role of macrophages in the clearance of inhaled ultrafine titanium dioxide particles. American Journal of Respiratory Cell and Molecular Biology, 38(3), 371376. https://doi.org/10.1165/rcmb.2007-0138OC

Goodman, Y., Bruce, A. J., Cheng, B., \& Mattson, M. P. (1996). Estrogens Attenuate and Corticosterone Exacerbates Excitotoxicity, Oxidative Injury, and Amyloid $\beta$-Peptide Toxicity in Hippocampal Neurons. Journal of Neurochemistry, 66(5), 1836-1844. 
https://doi.org/10.1046/j.1471-4159.1996.66051836.x

Grissa, I., Guezguez, S., Ezzi, L., Chakroun, S., Sallem, A., Kerkeni, E., ... Haouas, Z. (2016). The effect of titanium dioxide nanoparticles on neuroinflammation response in rat brain. Environmental Science and Pollution Research, 23, 20205-20213. https://doi.org/10.1007/s11356-016-7234-8

Guo, D., Xie, G., \& Luo, J. (2014). Mechanical properties of nanoparticles: Basics and applications. Journal of Physics D: Applied Physics, 47(1), 1-25. https://doi.org/10.1088/0022-3727/47/1/013001

Guxens, M., Lubczyńska, M. J., Muetzel, R. L., Dalmau-Bueno, A., Jaddoe, V. W. V., Hoek, G., ... El Marroun, H. (2018). Air Pollution Exposure During Fetal Life, Brain Morphology, and Cognitive Function in School-Age Children. Biological Psychiatry, 1-9. https://doi.org/10.1016/j.biopsych.2018.01.016

Hendren, C. O., Mesnard, X., Dröge, J., \& Wiesner, M. R. (2011). Estimating production data for five engineered nanomaterials as a basis for exposure assessment. Environmental Science and Technology, 45, 2562-2569. https://doi.org/10.1021/es103300g

Hong, F., Sheng, L., Ze, Y., Hong, J., Zhou, Y., Wang, L., .. Ze, X. (2015). Suppression of neurite outgrowth of primary cultured hippocampal neurons is involved in impairment of glutamate metabolism and NMDA receptor function caused by nanoparticulate TiO<inf $>2<$ inf $>$. Biomaterials, 53, 76-85. https://doi.org/10.1016/j.biomaterials.2015.02.067

Hong, F., Zhou, Y., Ji, J., Zhuang, J., Sheng, L., \& Wang, L. (2018). Nano-TiO2 Inhibits Development of the Central Nervous System and Its Mechanism in Offspring Mice. Journal 
of Agricultural and Food Chemistry, 66(44), 11767-11774.

https://doi.org/10.1021/acs.jafc.8b02952

Hougaard, Karin S, Jackson, P., Jensen, K. a, Sloth, J. J., Löschner, K., Larsen, E. H., ... Vogel, U. (2010). Effects of prenatal exposure to surface-coated nanosized titanium dioxide (UVTitan). A study in mice. Particle and Fibre Toxicology, 7, 16. https://doi.org/10.1186/1743$8977-7-16$

Hougaard, Karin Sørig, Campagnolo, L., Chavatte-Palmer, P., Tarrade, A., Rousseau-Ralliard, D., Valentino, S., ... Cassee, F. R. (2015). A perspective on the developmental toxicity of inhaled nanoparticles. Reproductive Toxicology, 56, 118-140.

https://doi.org/10.1016/j.reprotox.2015.05.015

Hu, R., Gong, X., Duan, Y., Li, N., Che, Y., Cui, Y., ... Hong, F. (2010). Neurotoxicological effects and the impairment of spatial recognition memory in mice caused by exposure to TiO2 nanoparticles. Biomaterials, 31, 8043-8050.

https://doi.org/10.1016/j.biomaterials.2010.07.011

Jackson, P., Vogel, U., Wallin, H., \& Hougaard, K. S. (2011). Prenatal Exposure to Carbon Black (Printex 90): Effects on Sexual Development and Neurofunction. Basic \& Clinical Pharmacology \& Toxicology, 109(6), 434-437. https://doi.org/10.1111/j.17427843.2011.00745.x

Jani, P., Halbert, G. W., Langridge, J., \& Florence, A. T. (1990). Nanoparticle Uptake By the Rat Gastrointestinal Mucosa - Quantitation and Particle-Size Dependency. Journal of Pharmacy and Pharmacology, 42(12), 821-826.

Jay, T. M., \& Witter, M. P. (1991). Distribution of Hippocampal CA1 and Subicular Efferents in 
the Prefrontal Cortex of the Rat Studied by Means of Anterograde Transport of P h a s e o h u u Z g ark- L euco agglu t in in. Journal of Comparative Neurology, 313, 574-586.

Keaney, J. F., Shwaery, G. T., Xu, A., Nicolosi, R. J., Loscalzo, J., Foxall, T. L., \& Vita, J. A. (1994). 17ß-Estradiol preserves endothelial vasodilator function and limits low- density lipoprotein oxidation in hypercholesterolemic swine. Circulation, 89(5), 2251-2259. https://doi.org/10.1161/01.CIR.89.5.2251

Kern, J. K., Geier, D. A., Homme, K. G., King, P. G., Bjørklund, G., Chirumbolo, S., \& Geier, M. R. (2017). Developmental neurotoxicants and the vulnerable male brain: A systematic review of suspected neurotoxicants that disproportionally affect males. Acta Neurobiologiae Experimentalis, 77(4), 269-296.

Kim, E.-M., Palmer, P., Howard, V., Elsaesser, A., Taylor, A., Staats, G., \& O 'hare, E. (2013). Effect of Intracerebroventricular Injection of TiO 2 Nanoparticles on Complex Behaviour in the Rat. Journal of Nanoscience and Nanotechnology, 13(12), 8325-8330. https://doi.org/10.1166/jnn.2013.8217

Kimiko, H., Akira, Y., \& Masayasu, I. (1987). Effect of sex hormones on the fate of methylmercury and on glutathione metabolism in mice. Biochemical Pharmacology, 36(12), 1919-1924. https://doi.org/10.1016/0006-2952(87)90489-8

Klocke, C., Allen, J. L., Sobolewski, M., Blum, J. L., Zelikoff, J. T., \& Cory-Slechta, D. A. (2018). Exposure to fine and ultrafine particulate matter during gestation alters postnatal oligodendrocyte maturation, proliferation capacity, and myelination. NeuroToxicology, 65, 196-206. https://doi.org/10.1016/j.neuro.2017.10.004

Klocke, C., Allen, J. L., Sobolewski, M., Mayer-Pröschel, M., Blum, J. L., Lauterstein, D., ... 
Cory-Slechta, D. A. (2017). Neuropathological consequences of gestational exposure to concentrated ambient fine and ultrafine particles in the mouse. Toxicological Sciences, 156(2), 492-508. https://doi.org/10.1093/toxsci/kfx010

Krakauer, J. W., Ghazanfar, A. A., Gomez-Marin, A., MacIver, M. A., \& Poeppel, D. (2017). Neuroscience Needs Behavior: Correcting a Reductionist Bias. Neuron, 93(3), 480-490. https://doi.org/10.1016/j.neuron.2016.12.041

Kreyling, W. G., Holzwarth, U., Ã, C. S., Hirn, S., Sch, M., Lipka, J., ... Gibson, N. (2017). Quantitative biokinetics of titanium dioxide nanoparticles after oral application in rats : Part 2. Nanotoxicoloty, 11(4), 443-453.

Laties, V. G. (1978). How operant conditioning can contribute to behavioral toxicology. Environmental Health Perspectives, 26(October), 29-35.

Lattal, K A, \& Williams, A. M. (2006). Body weight and response acquisition with delayed reinforcement. Journal of the Experimental Analysis of Behavior, 67(1), 131-143. https://doi.org/10.1901/jeab.1997.67-131

Lattal, Kennon A., \& Gleeson, S. (1990). Response acquisition with delayed reinforcement. Journal of Experimental Psychology: Animal Behavior Processes, 16(1), 27-39. https://doi.org/10.1037/0097-7403.16.1.27

Lavoie, J.-C., \& Chessex, P. (1997). Gender and maturation affect glutathione status in human neonatal tissues. Free Radical Biology and Medicine, 23, 648-657.

LeSage, M. G., Byrne, T., \& Poling, A. (1996). Effects of D-amphetamine on response aquisition with immediate and delayed reinforcement. Journal of the Experimental Analysis of 
Behavior, 3(3), 349-367. https://doi.org/10.1006/excr.1996.0116

Long, T. C., Tajuba, J., Sama, P., Saleh, N., Swartz, C., Parker, J., .. Veronesi, B. (2007). Nanosize titanium dioxide stimulates reactive oxygen species in brain microglia and damages neurons in vitro. Environmental Health Perspectives, 115(11), 1631-1637. https://doi.org/10.1289/ehp.10216

Losert, S., Von Goetz, N., Bekker, C., Fransman, W., Wijnhoven, S. W. P., Delmaar, C., ... Ulrich, A. (2014). Human exposure to conventional and nanoparticle-containing sprays - A critical review. Environmental Science and Technology, 48(10), 5366-5378. https://doi.org/10.1021/es5001819

Marquis, J. P., Goulet, S., \& Doré, F. Y. (2008). Neonatal ventral hippocampus lesions disrupt extra-dimensional shift and alter dendritic spine density in the medial prefrontal cortex of juvenile rats. Neurobiology of Learning and Memory, 90(2), 339-346. https://doi.org/10.1016/j.nlm.2008.04.005

McAlonan, K., \& Brown, V. J. (2003). Orbital prefrontal cortex mediates reversal learning and not attentional set shifting in the rat. Behavioural Brain Research, 146, 97-103. https://doi.org/10.1016/j.bbr.2003.09.019

Mehta, A., Prabhakar, M., Kumar, P., Deshmukh, R., \& Sharma, P. L. (2013). Excitotoxicity: Bridge to various triggers in neurodegenerative disorders. European Journal of Pharmacology, 698(1-3), 6-18. https://doi.org/10.1016/j.ejphar.2012.10.032

Mendelowitsch, A., Ritz, M. F., Ros, J., Langemann, H., \& Gratzl, O. (2001). 17ß-Estradiol reduces cortical lesion size in the glutamate excitotoxicity model by enhancing extracellular lactate: A new neuroprotective pathway. Brain Research, 901(1-2), 230-236. 
https://doi.org/10.1016/S0006-8993(01)02359-9

Min, J. young, \& Min, K. bok. (2017). Exposure to ambient PM10and NO2and the incidence of attention-deficit hyperactivity disorder in childhood. Environment International, 99(2), 221-227. https://doi.org/10.1016/j.envint.2016.11.022

Mohammadipour, A., Fazel, A., Haghir, H., Motejaded, F., Rafatpanah, H., Zabihi, H., ... Bideskan, A. E. (2014). Maternal exposure to titanium dioxide nanoparticles during pregnancy; impaired memory and decreased hippocampal cell proliferation in rat offspring. Environmental Toxicology and Pharmacology, 37(2), 617-625. https://doi.org/10.1016/j.etap.2014.01.014

Montazer, M., \& Seifollahzadeh, S. (2011). Enhanced self-cleaning, antibacterial and UV protection properties of nano $\mathrm{TiO} 2$ treated textile through enzymatic pretreatment. Photochemistry and Photobiology, 87, 877-883. https://doi.org/10.1111/j.17511097.2011.00917.x

Monteiro-Riviere, N. A., Wiench, K., Landsiedel, R., Schulte, S., Inman, A. O., \& Riviere, J. E. (2011). Safety evaluation of sunscreen formulations containing titanium dioxide and zinc oxide nanoparticles in UVB sunburned skin: An In vitro and in vivo study. Toxicological Sciences, 123(1), 264-280. https://doi.org/10.1093/toxsci/kfr148

Morris-Schaffer, K., Merrill, A., Jew, K., Wong, C., Conrad, K., Harvey, K., ... Cory-slechta, D. A. (2019). Effects of neonatal inhalation exposure to ultrafine carbon particles on pathology and behavioral outcomes in C57BL / 6J mice. Particle and Fibre Toxicology, 5, 1-15.

Morris-Schaffer, K., Merrill, A. K., Wong, C., Jew, K., Sobolewski, M., \& Cory-slechta, D. A. (2019). Limited developmental neurotoxicity from neonatal inhalation exposure to diesel 
exhaust particles in C57BL / 6 mice. Particle and Fibre Toxicology, 8, 1-14.

Morris-Schaffer, K., Sobolewski, M., Welle, K., Conrad, K., Yee, M., Reilly, M. A. O., \& Coryslechta, D. A. (2018). Cognitive flexibility deficits in male mice exposed to neonatal hyperoxia followed by concentrated ambient ultra fi ne particles. Neurotoxicology and Teratology, 70(October 2018), 51-59. https://doi.org/10.1016/j.ntt.2018.10.003

Mueller, N. C., \& Nowack, B. (2008). Exposure modelling of engineered nanoparticles in the environment. Environmental Science \& Technology, 42(12), 44447-44453. https://doi.org/10.1021/es7029637

Murphy, M. P. (2008). How mitochondria produce reactive oxygen species. Biochemical Journal, 417(1), 1-13. https://doi.org/10.1042/bj20081386

Nel, A., Xia, T., Madler, L., \& Li, N. (2006). Toxic potential of materials at the nanolevel. Science, 311(5761), 622-627. https://doi.org/10.1126/science.1114397

Newland, C. M. (2010). Neural, Behavioral, and Measurement Considerations in the Detection of Motor Impairment. In C. A. McQueen (Ed.), Comprehensive Toxicology (2nd ed., pp. 289-313). Elsevier Science.

Notter, T., Aengenheister, L., Weber-Stadlbauer, U., Naegeli, H., Wick, P., Meyer, U., \& Buerki-Thurnherr, T. (2018). Prenatal exposure to TiO2 nanoparticles in mice causes behavioral deficits with relevance to autism spectrum disorder and beyond. Translational Psychiatry, 8(1). https://doi.org/10.1038/s41398-018-0251-2

Oberdörster, Günter, Finkelstein, Jacob, Johnston, Carl, Gelein, Robert, Cox, Christopher, Baggs, Raymond, \& Elder, A. (2000). Acute pulmonary effects of ultrafine particles in rats 
and mice. Research Report: Health Effects Institute, (96), 5-74.

Oberdörster, G., Elder, A., \& Rinderknecht, A. (2009). Nanoparticles and the Brain: Cause for Concern? Journal of Nanoscience and Nanotechnology, 9, 4996-5007.

Oberdörster, G., Oberdörster, E., \& Oberdörster, J. (2005). Nanotoxicology: An emerging discipline evolving from studies of ultrafine particles. Environmental Health Perspectives, 113(7), 823-839. https://doi.org/10.1289/ehp.7339

Oberdörster, G., Sharp, Z., Atudorei, V., Elder, A., Gelein, R., Kreyling, W., \& Cox, C. (2004). Translocation of inhaled ultrafine particles to the brain. Inhalation Toxicology, 16(6-7), 437-445. https://doi.org/10.1080/08958370490439597

Paletz, E. M., Craig-Schmidt, M. C., \& Newland, M. C. (2006). Gestational exposure to methylmercury and n-3 fatty acids: Effects on high- and low-rate operant behavior in adulthood. Neurotoxicology and Teratology, 28(1), 59-73. https://doi.org/10.1016/j.ntt.2005.11.003

Paletz, E. M., Day, J. J., Craig-Schmidt, M. C., \& Newland, M. C. (2007). Spatial and visual discrimination reversals in adult and geriatric rats exposed during gestation to methylmercury and n - 3 polyunsaturated fatty acids. NeuroToxicology, 28(4), 707-719. https://doi.org/10.1016/j.neuro.2007.05.001

Palmer, H. J., \& Paulson, K. E. (1997). Reactive Oxygen Species and Antioxidants in Signal Transduction and Gene Expression. Nutrition Reviews, 55, 353-361. https://doi.org/10.1111/j.1753-4887.1997.tb01561.x

Park, J., Ham, S., Jang, M., Lee, J., Kim, S., Kim, S., .. Yoon, C. (2017). Spatial-Temporal 
Dispersion of Aerosolized Nanoparticles during the Use of Consumer Spray Products and Estimates of Inhalation Exposure. Environmental Science and Technology, 51(13), 76247638. https://doi.org/10.1021/acs.est.7b00211

Pathakoti, K., Manubolu, M., \& Hwang, H. M. (2017). Nanostructures: Current uses and future applications in food science. Journal of Food and Drug Analysis, 25(2), 245-253. https://doi.org/10.1016/j.jfda.2017.02.004

Pattij, T., Janssen, M. C. W., Vanderschuren, L. J. M. J., Schoffelmeer, A. N. M., \& van Gaalen, M. M. (2007). Involvement of dopamine D1 and D2 receptors in the nucleus accumbens core and shell in inhibitory response control. Psychopharmacology, 191(3), 587-598. https://doi.org/10.1007/s00213-006-0533-x

Payne-Sturges, D. C., Marty, M. A., Perera, F., Miller, M. D., Swanson, M., Ellickson, K., ... Hertz-Picciotto, I. (2019). Healthy air, healthy brains: Advancing air pollution policy to protect children's health. American Journal of Public Health, 109, 550-554. https://doi.org/10.2105/AJPH.2018.304902

Petitot, F., Lestaevel, P., Tourlonias, E., Mazzucco, C., Jacquinot, S., Dhieux, B., ... Dublineau, I. (2013). Inhalation of uranium nanoparticles: Respiratory tract deposition and translocation to secondary target organs in rats. Toxicology Letters, 217(3), 217-225. https://doi.org/10.1016/j.toxlet.2012.12.022

Piccinno, F., Gottschalk, F., Seeger, S., \& Nowack, B. (2012). Industrial production quantities and uses of ten engineered nanomaterials in Europe and the world. Journal of Nanoparticle Research, 14(9). https://doi.org/10.1007/s11051-012-1109-9

Placek, K., Dippel, W. C., Jones, S., \& Brady, A. M. (2013). Impairments in set-shifting but not 
reversal learning in the neonatal ventral hippocampal lesion model of schizophrenia :

Further evidence for medial prefrontal deficits. Behavioural Brain Research, 256, 405-413. https://doi.org/10.1016/j.bbr.2013.08.034

Pujalté, I., Dieme, D., Haddad, S., Serventi, A. M., \& Bouchard, M. (2017). Toxicokinetics of titanium dioxide (TiO2) nanoparticles after inhalation in rats. Toxicology Letters, 265, 7785. https://doi.org/10.1016/j.toxlet.2016.11.014

Randall, R., \& Thayer, S. (2018). Glutamate-induced calcium transient triggers delayed calcium overload and neurotoxicity in rat hippocampal neurons. The Journal of Neuroscience, 12(5), 1882-1895. https://doi.org/10.1523/jneurosci.12-05-01882.1992

Reed, M. N., Paletz, E. M., \& Newland, M. C. (2006). Gestational exposure to methylmercury and selenium: Effects on spatial discrimination reversal in adulthood. Neurotoxicology, 27(5), 721-732.

Reimhult, E. (2015). Nanoparticle-triggered release from lipid membrane vesicles. New Biotechnology, 32(6), 665-672. https://doi.org/10.1016/j.nbt.2014.12.002

Rice. (1992). Behavioral effects of lead in monkeys tested during infancy and adulthood. Neurotoxicology and Teratology, 14(4), 235-245. https://doi.org/10.1016/08920362(92)90002-R

Rice. (1998). Effects of postnatal exposure to a PCB mixture in monkeys on nonspatial discrimination reversal and delayed alternation performance. Neurotoxicology, 20, 391-400. Retrieved from http://www.ncbi.nlm.nih.gov/pubmed/9291496

Rice, D., \& Barone, S. (2000). Critical periods of vulnerability for the developing nervous 
system: Evidence from humans and animal models. Environmental Health Perspectives, 108(SUPPL. 3), 511-533. https://doi.org/10.1289/ehp.00108s3511

Rice, \& Gilbert. (1985). Low lead exposure from birth produces behavioral toxicity (DRL) in monkeys. Toxicology and Applied Pharmacology, 80, 421-426. https://doi.org/10.1016/0041-008X(85)90386-2

Richards, J. B., Sabol, K. E., \& Seiden, L. S. (1993). DRL interresponse-time distributions: Quantification by peak deviation analysis. Journal of the Ex, 60(2), 361-385.

Robbins, T. W. (2002). The 5-choice serial reaction time task: Behavioural pharmacology and functional neurochemistry. Psychopharmacology, 163(3-4), 362-380. https://doi.org/10.1007/s00213-002-1154-7

Robichaud, C. O., Uyar, A. E., Darby, M. R., Zucker, L. G., \& Wiesner, M. R. (2009). Estimates of Upper Bounds and Trends in Nano-TiO2Production As a Basis for Exposure Assessment. Environmental Science \& Technology, 43(12), 4227-4233. https://doi.org/10.1021/es8032549

Rush, J. W. E., \& Sandiford, S. D. (2003). Plasma glutathione peroxidase in healthy young adults: Influence of gender and physical activity. Clinical Biochemistry, 36(5), 345-351. https://doi.org/10.1016/S0009-9120(03)00039-0

Ruszkiewicz, J. A., Bowman, A. B., Farina, M., Rocha, J. B. T., \& Aschner, M. (2016). Sex- and structure-specific differences in antioxidant responses to methylmercury during early development. NeuroToxicology, 56, 118-126. https://doi.org/10.1016/j.neuro.2016.07.009

Sable, H. J. K., Eubig, P. A., Powers, B. E., Wang, V. C., \& Schantz, S. L. (2009). 
Developmental exposure to PCBs and/or MeHg: Effects on a differential reinforcement of low rates (DRL) operant task before and after amphetamine drug challenge.

Neurotoxicology and Teratology, 31(3), 149-158. https://doi.org/10.1016/j.ntt.2008.12.006

Sadler, T. W. (2005). Embryology of neural tube development. American Journal of Medical Genetics - Seminars in Medical Genetics, 135 C, 2-8. https://doi.org/10.1002/ajmg.c.30049

Sánchez-Cardoso, P., Higuera-Matas, A., Martín, S., Miguéns, M., Del Olmo, N., GarcíaLecumberri, C., \& Ambrosio, E. (2009). Strain differences between Lewis and Fischer 344 rats in the modulation of dopaminergic receptors after morphine self-administration and during extinction. Neuropharmacology, 57(1), 8-17. https://doi.org/10.1016/j.neuropharm.2009.03.014

Sattler, R., \& Tymianski, M. (2000). Molecular mechanisms of calcium-dependent excitotoxicity. Journal of Molecular Medicine (Berlin, Germany), 78(1), 3-13. Retrieved from http://www.ncbi.nlm.nih.gov/pubmed/10759025

Semmler, M., Seitz, J., Erbe, F., Mayer, P., Heyder, J., Oberdörster, G., \& Kreyling, W. G. (2004). Long-term clearance kinetics of inhaled ultrafine insoluble iridium particles from the rat lung, including transient translocation into secondary organs. Inhalation Toxicology, 16(6-7), 453-459. https://doi.org/10.1080/08958370490439650

Shimizu, M., Tainaka, H., Oba, T., Mizuo, K., Umezawa, M., \& Takeda, K. (2009). Maternal exposure to nanoparticulate titanium dioxide during the prenatal period alters gene expression related to brain development in the mouse. Particle and Fibre Toxicology, 6, 29. https://doi.org/10.1186/1743-8977-6-20

Shinohara, Y. (2012). Quantification of postsynaptic density proteins: Glutamate receptor 
subunits and scaffolding proteins. Hippocampus, 22(5), 942-953.

https://doi.org/10.1002/hipo.20950

Simpkins, J. W., Rajakumar, G., Zhang, Y.-Q., Simpkins, C. E., Greenwald, D., Yu, C. J., ...

Day, A. L. (1997). Estrogens may reduce mortality and ischemic damage caused by middle cerebral artery occlusion in the female rat. Journal of Neurosurgery, 87(5), 724-730. https://doi.org/10.3171/jns.1997.87.5.0724

Sinden, J. D., Rawlins, J. N. P., Gray, J. A., \& Jarrard, L. E. (1986). Selective Cytotoxic Lesions of the Hippocampal Formation and DRL Performance in Rats. Behavioral Neuroscience, 100(3), 320-329. https://doi.org/10.1037/0735-7044.100.3.320

Skocaj, M., Filipic, M., Petkovic, J., \& Novak, S. (2011). Titanium dioxide in our everyday life; Is it safe? Radiology and Oncology, 45(4), 227-247. https://doi.org/10.2478/v10019-011$0037-0$

Srinivas, P. R., Philbert, M., Vu, T. Q., Huang, Q., Kokini, J. L., Saos, E., ... Ross, S. a. (2010). Nanotechnology research: Applications in nutritional sciences. The Journal of Nutrition, 140, 119-124. https://doi.org/10.3945/jn.109.115048.119

Stapleton, P A, Hathaway, Q. A., Nichols, C. E., Abukabda, A. B., Pinti, M. V, Shepherd, D. L., ... Nurkiewicz, T. R. (2018). Maternal engineered nanomaterial inhalation during gestation alters the fetal transcriptome. Particle and Fibre Toxicology, 15, 1-15. https://doi.org/10.1186/s12989-017-0239-8

Stapleton, Phoebe A., Minarchick, V. C., Yi, J., Engels, K., McBride, C. R., \& Nurkiewicz, T. R. (2013). Maternal Engineered Nanomaterial Exposure and Fetal Microvascular Function: Does the Barker Hypothesis Apply? American Journal of Obstetrition and Gynecology, 
209(3), 1-24. https://doi.org/10.1016/j.ajog.2013.04.036.Maternal

Stark, M. J., Hodyl, N. A., Wright, I. M. R., \& Clifton, V. L. (2011). Influence of sex and glucocorticoid exposure on preterm placental pro-oxidant-antioxidant balance. Placenta, 32(11), 865-870. https://doi.org/10.1016/j.placenta.2011.08.010

Sunyer, J., \& Dadvand, P. (2019). Pre-natal brain development as a target for urban air pollution. Basic \& Clinical Pharmacology \& Toxicology, 1(August 2018), 1-8. https://doi.org/10.1111/bcpt.13226

Sunyer, J., Esnaola, M., Alvarez-Pedrerol, M., Forns, J., Rivas, I., López-Vicente, M., ... Querol, X. (2015). Association between Traffic-Related Air Pollution in Schools and Cognitive Development in Primary School Children: A Prospective Cohort Study. PLoS Medicine, 12(3), 1-24. https://doi.org/10.1371/journal.pmed.1001792

Takahashi, Y., Mizuo, K., Shinkai, Y., Oshio, S., \& Takeda, K. (2010). Prenatal exposure to titanium dioxide nanoparticles increases dopamine levels in the prefrontal cortex and neostriatum of mice. The Journal of Toxicological Sciences, 35(5), 749-756. https://doi.org/10.2131/jts.35.749

Takeda, K., Suzuki, K., Ishihara, A., Kubo-Irie, M., Fujimoto, R., Tabata, M., ... Sugamata, M. (2009). Nanoparticles Transferred from Pregnant Mice to Their Offspring Can Damage the Genital and Cranial Nerve Systems. Journal Of Health Science, 55(1), 95-102. https://doi.org/10.1248/jhs.55.95

Takenaka, S., Karg, E., Roth, C., Schulz, H., Ziesenis, A., Heinzmann, U., ... Heyder, J. (2001). Pulmonary and systemic distribution of inhaled ultrafine silver particles in rats. Environmental Health Perspectives, 109(SUPPL. 4), 547-551. 
https://doi.org/10.1289/ehp.01109s4547

Ujjain, S. K., Roy, P. K., Kumar, S., Singha, S., \& Khare, K. (2016). Uniting Superhydrophobic, Superoleophobic and Lubricant Infused Slippery Behavior on Copper Oxide Nanostructured Substrates. Scientific Reports, 6, 1-10. https://doi.org/10.1038/srep35524

Umezawa, M., Tainaka, H., Kawashima, N., Shimizu, M., \& Takeda, K. (2012). Effect of fetal exposure to titanium dioxide nanoparticle on brain development $\wedge^{\wedge} \wedge$ minus; brain region information. The Journal of Toxicological Sciences, 37(6), 1247-1252. https://doi.org/10.2131/jts.37.1247

Valavanidis, A., \& Fiotakis, K. (2008). Airborne Particulate Matter and Human Health : Toxicological Assessment and Importance of Size and Composition of Particles for Oxidative Damage and Carcinogenic Mechanisms. Journal of Environmental Science and Health Part C, 26, 339-362. https://doi.org/10.1080/10590500802494538

Vance, M. E., Kuiken, T., Vejerano, E. P., McGinnis, S. P., Hochella, M. F., \& Hull, D. R. (2015). Nanotechnology in the real world: Redeveloping the nanomaterial consumer products inventory. Beilstein Journal of Nanotechnology, 6(1), 1769-1780. https://doi.org/10.3762/bjnano.6.181

Verwer, R. W. H., Meijer, R. J., Van Uum, H. F. M., \& Witter, M. P. (1997). Collateral projections from the rat hippocampal formation to the lateral and medial prefrontal cortex. Hippocampus, 7(4), 397-402. https://doi.org/10.1002/(SICI)10981063(1997)7:4<397::AID-HIPO5>3.0.CO;2-G

Villa, A., Vegeto, E., Poletti, A., \& Maggi, A. (2016). Estrogens, neuroinflammation, and neurodegeneration. Endocrine Reviews, 37(4), 372-402. https://doi.org/10.1210/er.2016- 
1007

Volk, H. E., Lurmann, F., Penfold, B., Hertz-Picciotto, I., \& McConnell, R. (2013). TrafficRelated Air Pollution, Particulate Matter, and Autism. JAMA Psychiatry, 70(1), 71. https://doi.org/10.1001/jamapsychiatry.2013.266

Wang, J., Liu, Y., Jiao, F., Lao, F., Li, W., Gu, Y., ... Chen, C. (2008). Time-dependent translocation and potential impairment on central nervous system by intranasally instilled TiO2 nanoparticles. Toxicology, 254(1-2), 82-90. https://doi.org/10.1016/j.tox.2008.09.014

Warheit, D. B. (2013). How to measure hazards / risks following exposures to nanoscale or pigment-grade titanium dioxide particles. Toxicology Letters, 220(2), 193-204. https://doi.org/10.1016/j.toxlet.2013.04.002

Warheit, D. B., Overby, L. H., George, G., \& Brody, A. R. (1988). Pulmonary macrophages are attracted to inhaled particles through complement activation. Experimental Lung Research, 14(1), 51-66.

Wells, P. G., Bhuller, Y., Chen, C. S., Jeng, W., Kasapinovic, S., Kennedy, J. C., ... Wong, A. W. (2005). Molecular and biochemical mechanisms in teratogenesis involving reactive oxygen species. Toxicology and Applied Pharmacology, 207(2 SUPPL.), 354-366. https://doi.org/10.1016/j.taap.2005.01.061

Widholm, J. J., Clarkson, G. B., Strupp, B. J., Crofton, K. M., Seegal, R. F., \& Schantz, S. L. (2001). Spatial reversal learning in Aroclor 1254-exposed rats: Sex-specific deficits in associative ability and inhibitory control. Toxicology and Applied Pharmacology, 174, 188198. https://doi.org/10.1006/taap.2001.9199 
Widholm, J. J., Seo, B. W., Strupp, B. J., Seegal, R. F., \& Schantz, S. L. (2003). Effects of perinatal exposure to 2,3,7,8-tetrachlorodibenzo-p-dioxin on spatial and visual reversal learning in rats. Neurotoxicology and Teratology, 25, 459-471.

https://doi.org/10.1016/S0892-0362(03)00014-X

Wilkenfield, J., Nickel, M., Blakely, E., \& Poling, A. (1992). Acquisition of lever-press responding in rats with delayed reinforcement: A comparison of three procedures. Journal of the Experimental Analysis of Behavior, 58(3), 431-443. https://doi.org/10.1901/jeab.1992.58-431

Woodward, N. C., Haghani, A., Johnson, R. G., Hsu, T. M., Saffari, A., Sioutas, C., ... Finch, C. E. (2018). Prenatal and early life exposure to air pollution induced hippocampal vascular leakage and impaired neurogenesis in association with behavioral de fi cits. Translational Psychiatry, 1-10. https://doi.org/10.1038/s41398-018-0317-1

World Health Organization. (2017). Evolution of WHO air quality guidelines: past, present and future. Retrieved from http://www.euro.who.int/_data/assets/pdf_file/0019/331660/Evolution-airquality.pdf?ua=1

Yi, J., Chen, B. T., Schwegler-Berry, D., Frazer, D., Castranova, V., Mcbride, C., ... Nurkiewicz, T. R. (2013). Whole-body nanoparticle aerosol inhalation exposures. Journal of Visulalized Experiments, 75. https://doi.org/10.3791/50263

Ze, Y., Sheng, L., Zhao, X., Ze, X., Wang, X., Zhou, Q., ... Hong, F. (2014). Neurotoxic characteristics of spatial recognition damage of the hippocampus in mice following subchronic peroral exposure to TiO2 nanoparticles. Journal of Hazardous Materials, 264, 
219-229. https://doi.org/10.1016/j.jhazmat.2013.10.072

Zhang, L., Bai, R., Li, B., Ge, C., Du, J., Liu, Y., ... Chen, C. (2011). Rutile TiO2particles exert size and surface coating dependent retention and lesions on the murine brain. Toxicology Letters, 207(1), 73-81. https://doi.org/10.1016/j.toxlet.2011.08.001

Zhou, Y., Ji, J., Chen, C., \& Hong, F. (2019). Retardation of axonal and Dendritic Outgrowth Is Associated with the MAPK Signaling Pathway in Offspring Mice Following Maternal Exposure to Nanosized Titanium Dioxide [Research-article]. Journal of Agricultural and Food Chemistry, 67(9), 2709-2715. https://doi.org/10.1021/acs.jafc.8b06992

Zhou, Y., Ji, J., Hong, F., Zhuang, J., \& Wang, L. (2019). Maternal exposure to nanoparticulate titanium dioxide causes inhibition of hippocampal developmental involving dysfunction of the Rho/NMDAR signaling pathway in offspring. Journal of Biomedical Nanotechnology, $15,839-847$.

Zimmerman, J., \& Schuster, C. R. (1962). Spaced repsonding in multiple DRL schedules. Journal of the Experimental Analysis of Behavior, 5, 497-504. 
Table 1. ANOVA outcomes for Experiment 1

\begin{tabular}{|c|c|c|c|c|c|}
\hline Measure & Effect & $F$ & $d f$ & $p$ & $\eta_{\mathrm{p}}^{2}$ \\
\hline \multicolumn{6}{|l|}{ All rats } \\
\hline \multirow[t]{3}{*}{ Pellets earned } & Group & 0.46 & 1,25 & 0.50 & 0.02 \\
\hline & Sex & 1.94 & 1,25 & 0.18 & 0.07 \\
\hline & Group*Sex & 1.61 & 1,25 & 0.22 & 0.06 \\
\hline \multirow[t]{3}{*}{ Obtained delay } & Group & 0.15 & 1,25 & 0.69 & 0.06 \\
\hline & Sex & 0.23 & 1,25 & 0.64 & 0.09 \\
\hline & Group*Sex & 41.35 & 1,25 & $<0.01$ & 0.24 \\
\hline \multirow[t]{3}{*}{ Active responses } & Group & 0.11 & 1,25 & 0.75 & $<0.01$ \\
\hline & Sex & 1.87 & 1,25 & 0.18 & 0.07 \\
\hline & Group*Sex & 3.15 & 1,25 & 0.08 & 0.11 \\
\hline \multirow[t]{3}{*}{ Inactive responses } & Group & 0.06 & 1,25 & 0.93 & $<0.01$ \\
\hline & Sex & 3.70 & 1,25 & 0.06 & 0.13 \\
\hline & Group*Sex & 0.61 & 1,25 & 0.44 & 0.02 \\
\hline \multicolumn{6}{|l|}{$>20$ pellets } \\
\hline \multirow[t]{3}{*}{ Pellets earned } & Group & 5.88 & 1,11 & 0.03 & 0.35 \\
\hline & Sex & 0.12 & 1,11 & 0.73 & 0.01 \\
\hline & Group*Sex & 7.95 & 1,11 & 0.02 & 0.42 \\
\hline \multirow[t]{3}{*}{ Obtained delay } & Group & 0.64 & 1,11 & 0.44 & 0.05 \\
\hline & Sex & 0.83 & 1,11 & 0.38 & 0.07 \\
\hline & Group*Sex & 10.69 & 1,11 & $<0.01$ & 0.49 \\
\hline \multirow[t]{3}{*}{ Active responses } & Group & 0.42 & 1,11 & 0.53 & 0.04 \\
\hline & Sex & 0.01 & 1,11 & 0.91 & $<0.01$ \\
\hline & Group*Sex & 4.73 & 1,11 & 0.05 & 0.30 \\
\hline \multirow[t]{3}{*}{ Inactive responses } & Group & $<0.01$ & 1,11 & 0.99 & $<0.01$ \\
\hline & Sex & $<0.01$ & 1,11 & 0.99 & $<0.01$ \\
\hline & Group*Sex & 0.91 & 1,11 & 0.36 & 0.07 \\
\hline
\end{tabular}

Note. Bolded values indicate statistical significance $(p<0.05)$. 
Table 2. Linear regression outcomes for Experiment 2 - Males only

\begin{tabular}{|c|c|c|c|c|c|c|}
\hline Session 1 (R1-R4) & Effect & $B$ & Std Error & $d f$ & $t$ & $p$ \\
\hline Session to criterion & Group & 0.97 & 0.51 & 53.73 & 1.89 & 0.06 \\
\hline \multirow[t]{2}{*}{ Model R ${ }^{2}: 0.78$} & Reversal & -0.39 & 0.11 & 42.99 & 3.63 & $<0.01$ \\
\hline & Group*Reversal & -0.25 & 0.17 & 42.99 & 1.47 & 0.15 \\
\hline Total error & Group & 0.26 & 0.36 & 55.18 & 0.71 & 0.47 \\
\hline \multirow{2}{*}{ Model R2: 0.66} & Reversal & -0.73 & 0.09 & 42.99 & 7.53 & $<0.01$ \\
\hline & Group*Reversal & 0.46 & 0.13 & 42.99 & 0.34 & 0.72 \\
\hline Latency & Group & -0.99 & 0.51 & 31.03 & 1.95 & 0.06 \\
\hline \multirow{2}{*}{ Model $\mathrm{R}^{2}: 0.24$} & Reversal & -0.37 & 0.09 & 42.99 & 4.01 & $<0.01$ \\
\hline & Group*Reversal & 0.10 & 0.13 & 42.99 & 0.81 & 0.42 \\
\hline Error runs & Group & -1.04 & 0.56 & 55.95 & 1.86 & 0.06 \\
\hline \multirow{2}{*}{ Model $\mathrm{R}^{2}: 0.21$} & Reversal & -0.59 & 0.14 & 43 & 4.17 & $<0.01$ \\
\hline & Group*Reversal & 0.48 & 0.19 & 43 & 2.45 & 0.01 \\
\hline First run error & Group & 0.37 & 0.54 & 56 & 0.69 & 0.49 \\
\hline \multirow[t]{2}{*}{ Model $\mathrm{R}^{2}: 0.28$} & Reversal & -0.42 & 0.14 & 56 & 2.87 & $<0.01$ \\
\hline & Group*Reversal & -0.11 & 0.19 & 56 & 0.54 & 0.59 \\
\hline Error run length & Group & 1.12 & 0.58 & 54.84 & 0.68 & 0.056 \\
\hline \multirow[t]{2}{*}{ Model $\mathrm{R}^{2}: 0.21$} & Reversal & -0.17 & 0.15 & 43.03 & 1.10 & 0.27 \\
\hline & Group*Reversal & -0.35 & 0.21 & 43.03 & 1.67 & 0.10 \\
\hline Correct:stay ratio & Group & -0.49 & 0.37 & 56 & 1.35 & 0.18 \\
\hline \multirow[t]{2}{*}{ Model $\mathrm{R}^{2}: 0.67$} & Reversal & 0.68 & 0.09 & 56 & 6.96 & $<0.01$ \\
\hline & Group*Reversal & 0.07 & 0.13 & 56 & 0.54 & 0.59 \\
\hline Incorrect:shift ratio & Group & -0.86 & 0.57 & 56 & 1.50 & 0.14 \\
\hline \multirow[t]{2}{*}{ Model $\mathrm{R}^{2}: 0.22$} & Reversal & 0.24 & 0.15 & 56 & 1.60 & 0.11 \\
\hline & Group*Reversal & 0.27 & 0.21 & 56 & 1.31 & 0.19 \\
\hline
\end{tabular}


Session 2 (R1-R4)

\begin{tabular}{|c|c|c|c|c|c|c|}
\hline Total error & Group & 0.78 & 0.38 & 111.2 & 2.05 & 0.04 \\
\hline \multirow[t]{2}{*}{ Model $\mathrm{R}^{2}: 0.26$} & Reversal & -0.32 & 0.09 & 85 & 3.35 & $<0.01$ \\
\hline & Group*Reversal & -0.24 & 0.13 & 85 & 1.86 & 0.06 \\
\hline \multirow{3}{*}{$\begin{array}{r}\text { Latency } \\
\text { Model } \mathrm{R}^{2}: 0.31\end{array}$} & Group & -1.73 & 0.47 & 23.21 & 3.65 & $<0.01$ \\
\hline & Reversal & -0.41 & 0.07 & 43 & -5.75 & $<0.01$ \\
\hline & Group*Reversal & 0.32 & 0.09 & 43 & 3.30 & $<0.01$ \\
\hline \multirow{3}{*}{$\begin{array}{r}\text { Error runs } \\
\text { Model } \mathrm{R}^{2}: 0.18\end{array}$} & Group & 0.22 & 0.59 & 56 & 0.38 & 0.70 \\
\hline & Reversal & -0.36 & 0.15 & 56 & 2.29 & 0.02 \\
\hline & Group*Reversal & -0.03 & 0.21 & 56 & 0.18 & 0.86 \\
\hline \multirow{3}{*}{$\begin{array}{r}\text { First-run error } \\
\text { Model } \mathrm{R}^{2}: 0.22\end{array}$} & Group & 1.47 & 0.56 & 55.96 & 2.62 & 0.01 \\
\hline & Reversal & -0.08 & 0.14 & 43 & 0.62 & 0.54 \\
\hline & Group*Reversal & -0.46 & 0.19 & 43 & 2.31 & 0.02 \\
\hline \multirow{3}{*}{$\begin{array}{l}\text { Error run length } \\
\text { Model } \mathrm{R}^{2}: 0.08\end{array}$} & Group & 1.26 & 0.59 & 53.43 & 2.11 & 0.04 \\
\hline & Reversal & 0.12 & 0.14 & 43 & 0.83 & 0.41 \\
\hline & Group*Reversal & -0.46 & 0.19 & 43 & 2.34 & 0.02 \\
\hline \multirow{3}{*}{$\begin{array}{r}\text { Correct:stay ratio } \\
\text { Model } \mathrm{R}^{2}: 0.27\end{array}$} & Group & -0.94 & 0.53 & 53.58 & 1.78 & 0.08 \\
\hline & Reversal & 0.30 & 0.13 & 43 & 2.36 & 0.02 \\
\hline & Group*Reversal & 0.24 & 0.17 & 43 & 1.38 & 0.17 \\
\hline \multirow{3}{*}{$\begin{array}{r}\text { Incorrect:shift ratio } \\
\text { Model } R^{2}: 0.08\end{array}$} & Group & -1.09 & 0.60 & 55.39 & 1.81 & 0.07 \\
\hline & Reversal & -0.03 & 0.15 & 43 & 0.25 & 0.80 \\
\hline & Group*Reversal & 0.39 & 0.21 & 43 & 1.91 & 0.06 \\
\hline \multicolumn{7}{|l|}{ Reversal 1 (session 1-3) } \\
\hline & Group & 0.15 & 0.39 & 40.76 & 0.37 & 0.71 \\
\hline \multirow[t]{2}{*}{ Model $\mathrm{R}^{2}: 0.67$} & Session & -1.03 & 0.11 & 28 & 8.79 & $<0.01$ \\
\hline & Group*Session & 0.08 & 0.16 & 28 & 0.49 & 0.63 \\
\hline Correct:stay ratio & Group & -0.24 & 0.43 & 39.35 & 0.55 & 0.58 \\
\hline Model $\mathrm{R}^{2}: 0.58$ & Session & 0.94 & 0.12 & 28 & 7.73 & $<0.01$ \\
\hline
\end{tabular}




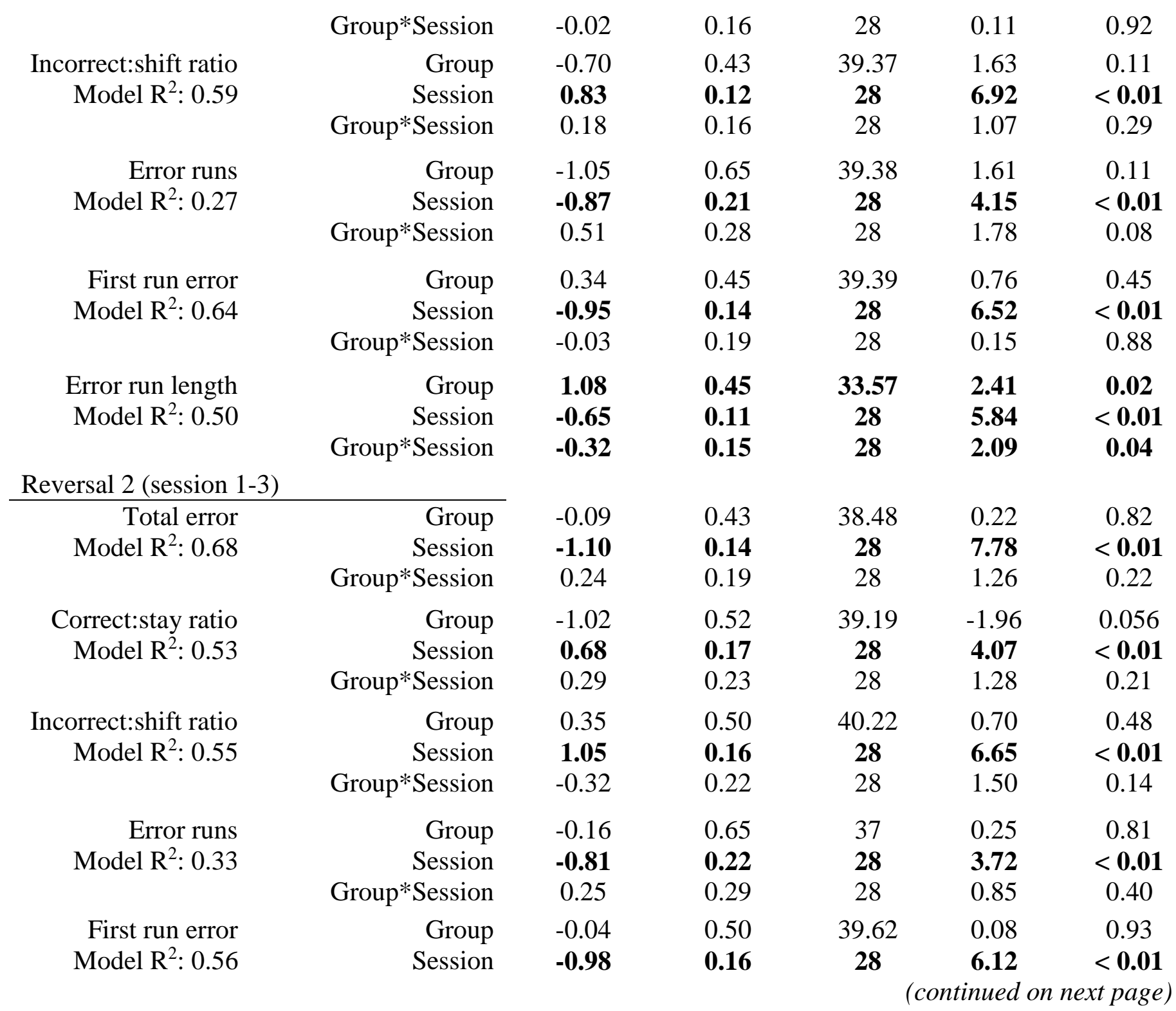




\begin{tabular}{rrccccc} 
& Group*Session & 0.15 & 0.22 & 28 & 0.69 & 0.49 \\
Error run length & Group & 0.01 & 0.54 & 39.68 & 0.01 & 0.98 \\
Model R R : 0.49 & Session & $\mathbf{- 0 . 9 2}$ & $\mathbf{0 . 1 7}$ & $\mathbf{2 8}$ & $\mathbf{5 . 3 7}$ & $<\mathbf{0 . 0 1}$ \\
& Group*Session & 0.13 & 0.23 & 28 & 0.53 & 0.59 \\
\hline
\end{tabular}

Note. Bolded values indicate statistical significance $(p<0.05)$. 
Table 3. Linear regression outcomes for Experiment 2 - Females only

\begin{tabular}{|c|c|c|c|c|c|c|}
\hline Session 1 (R1-R4) & Effect & B & Std Error & $d f$ & $t$ & $p$ \\
\hline \multirow{3}{*}{$\begin{array}{r}\text { Session to criterion } \\
\text { Model } \mathrm{R}^{2}: 0.58\end{array}$} & Group & 0.71 & 0.57 & 52 & 1.23 & 0.22 \\
\hline & Reversal & -0.36 & 0.16 & 52 & 2.30 & 0.02 \\
\hline & Group*Reversal & -0.14 & 0.21 & 52 & 0.67 & 0.50 \\
\hline & Group & -0.52 & 0.43 & 52 & 1.22 & 0.23 \\
\hline \multirow{2}{*}{ Model $\mathrm{R}^{2}: 0.58$} & Reversal & -0.83 & 0.12 & 52 & 7.01 & $<0.01$ \\
\hline & Group*Reversal & 0.29 & 0.15 & 52 & 1.86 & 0.06 \\
\hline \multirow{3}{*}{$\begin{array}{r}\text { Latency } \\
\text { Model } \mathrm{R}^{2}: 0.09\end{array}$} & Group & 0.16 & 0.585 & 27 & 1.27 & 0.21 \\
\hline & Reversal & -0.18 & 0.10 & 40 & 1.76 & 0.08 \\
\hline & Group*Reversal & -0.13 & 0.14 & 40 & 0.93 & 0.36 \\
\hline Error runs & Group & -0.77 & 0.57 & 52 & 1.35 & 0.18 \\
\hline \multirow[t]{2}{*}{ Model $\mathrm{R}^{2}: 0.26$} & Reversal & -0.63 & 0.15 & 40 & 4.09 & $<0.01$ \\
\hline & Group*Reversal & 0.35 & 0.20 & 40 & 1.72 & 0.09 \\
\hline First-run error & Group & 0.02 & 0.60 & 52 & 0.03 & 0.97 \\
\hline \multirow[t]{2}{*}{ Model $\mathrm{R}^{2}: 0.20$} & Reversal & -0.41 & 0.16 & 52 & 2.47 & 0.01 \\
\hline & Group*Reversal & 0.02 & 0.22 & 52 & 0.01 & 0.99 \\
\hline Error run length & Group & 0.11 & 0.62 & 52 & 0.17 & 0.86 \\
\hline \multirow[t]{2}{*}{ Model R $\mathrm{R}^{2}: 0.12$} & Reversal & -0.32 & 0.16 & 40 & 2.00 & 0.05 \\
\hline & Group*Reversal & 0.02 & 0.21 & 40 & 0.08 & 0.93 \\
\hline Correct:stay ratio & Group & 0.83 & 0.44 & 51.87 & 1.87 & 0.06 \\
\hline \multirow[t]{2}{*}{ Model $\mathrm{R}^{2}: 0.55$} & Reversal & 0.84 & 0.12 & 40 & 7.07 & $<0.01$ \\
\hline & Group*Reversal & -0.34 & 0.16 & 40 & 2.15 & 0.04 \\
\hline Incorrect:shift ratio & Group & -0.01 & 0.61 & 52 & 0.02 & 0.98 \\
\hline \multirow[t]{3}{*}{ Model $\mathrm{R}^{2}: 0.16$} & Reversal & 0.38 & 0.16 & 40 & 2.35 & 0.02 \\
\hline & Group*Reversal & -0.03 & 0.21 & 40 & 0.13 & 0.89 \\
\hline & & & & \multicolumn{3}{|c|}{ (continued on next page) } \\
\hline
\end{tabular}


Session 2 (R1-R4)

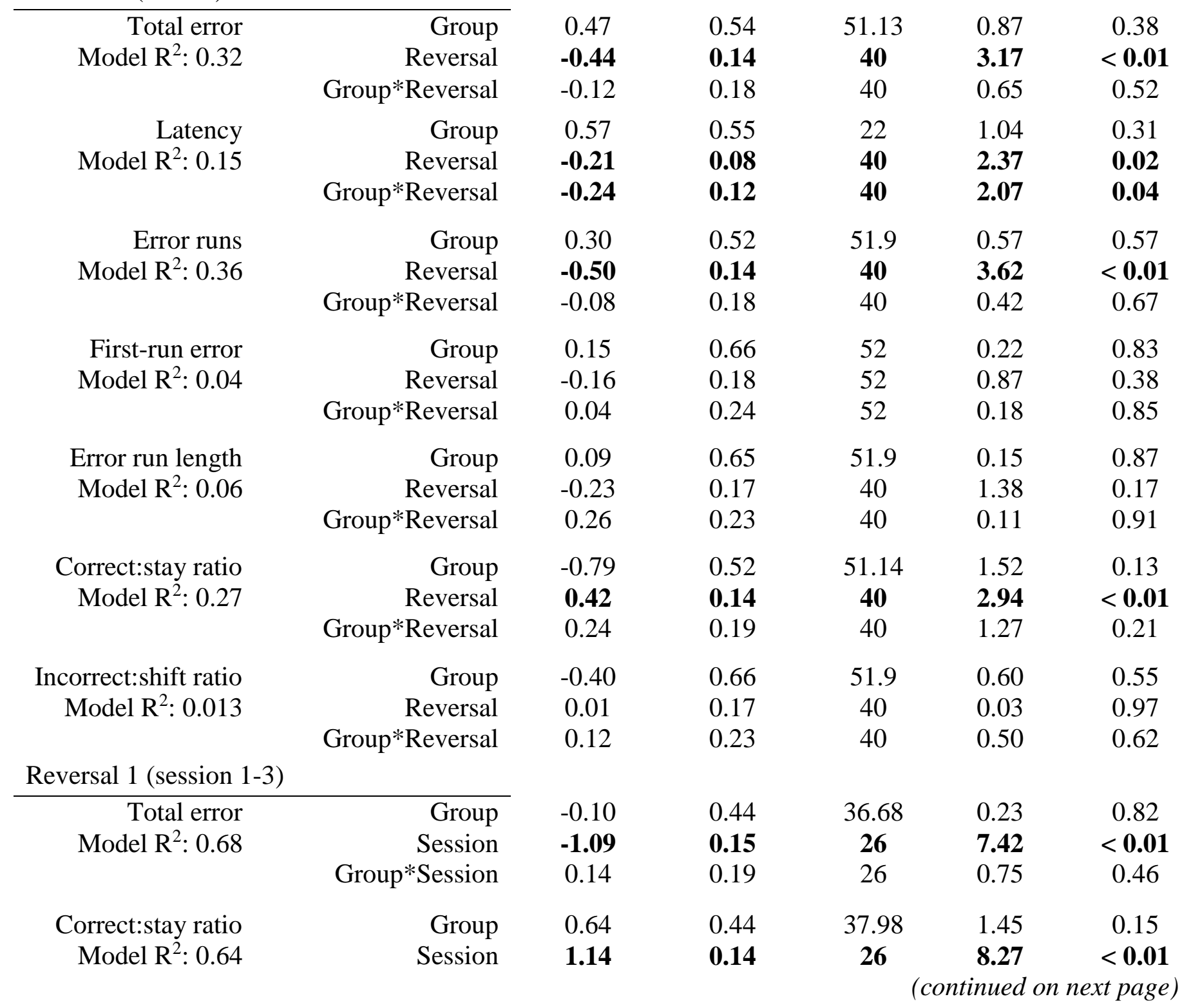




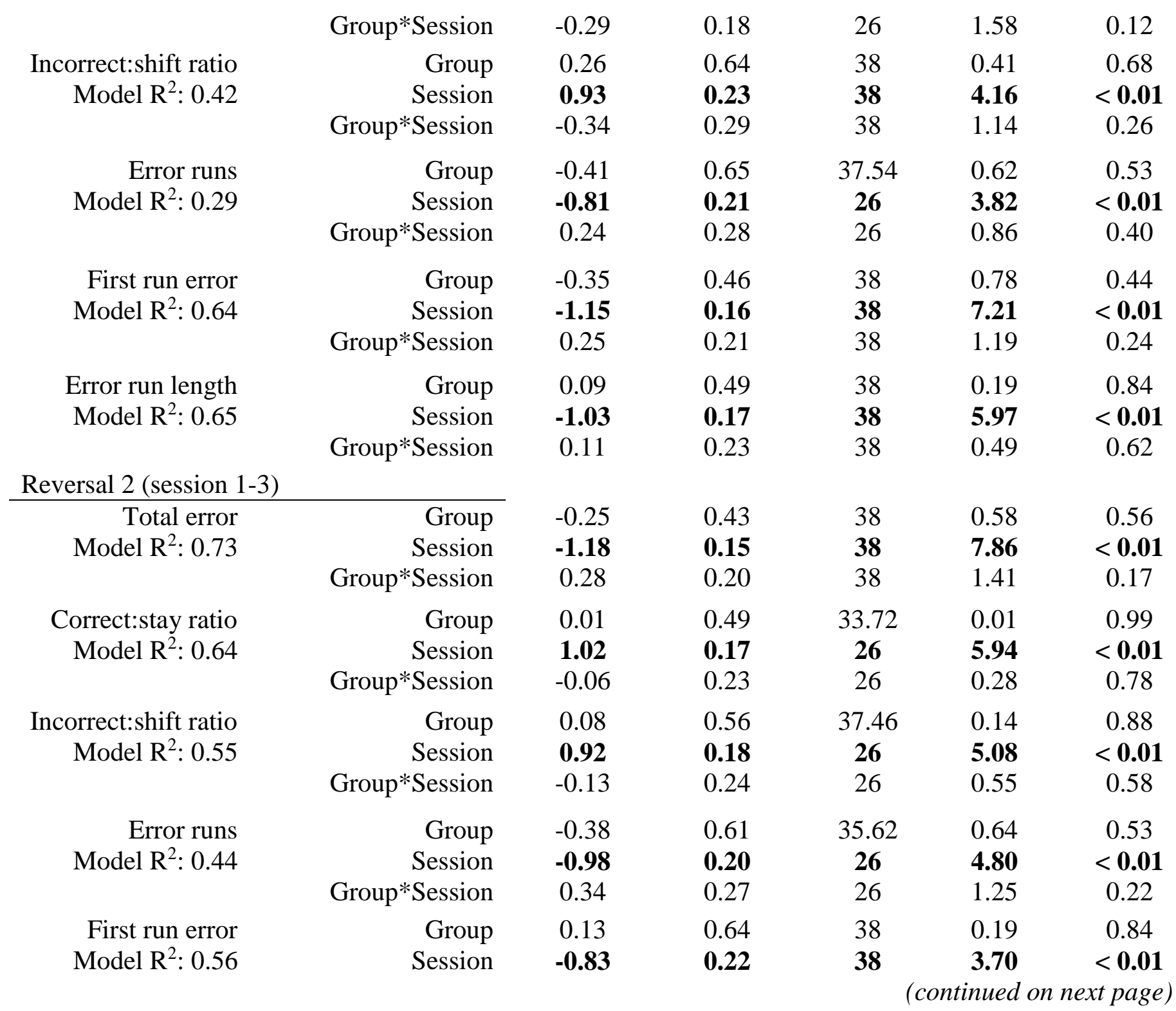




$\begin{array}{rrrrccc} & \text { Group*Session } & 0.08 & 0.30 & 38 & 0.28 & 0.78 \\ \text { Error run length } & \text { Group } & -0.17 & 0.61 & 37.56 & 0.28 & 0.78 \\ \text { Model R }{ }^{2}: 0.49 & \text { Session } & \mathbf{- 0 . 8 6} & \mathbf{0 . 2 0} & \mathbf{2 6} & \mathbf{4 . 3 4} & <\mathbf{0 . 0 1} \\ & \text { Group*Session } & 0.22 & 0.26 & 26 & 0.85 & 0.40\end{array}$

Note. Bolded values indicate statistical significance $(p<0.05)$. 
Table 4. Linear regression outcomes for Experiment 3 - Both Sexes

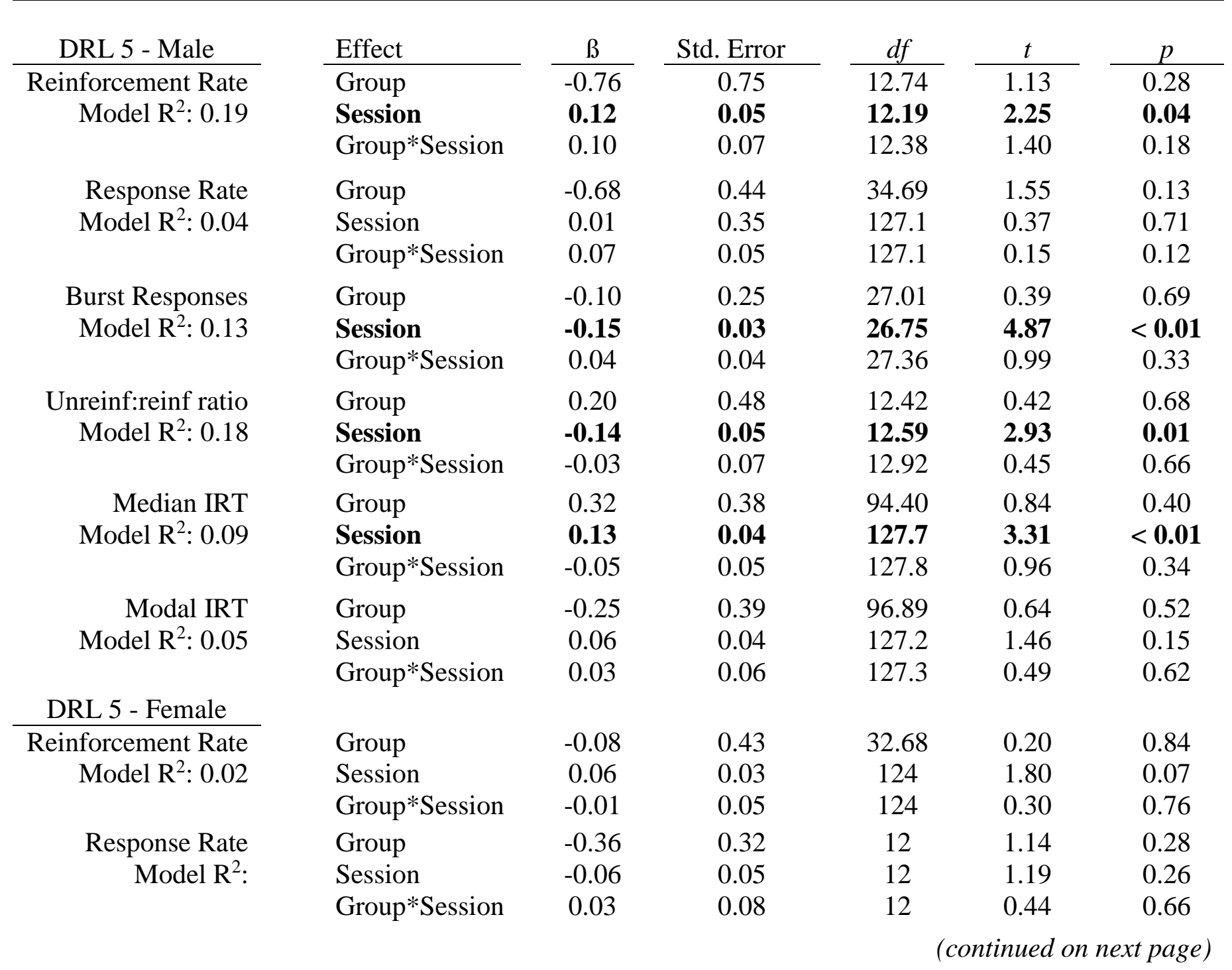




\begin{tabular}{|c|c|c|c|c|c|c|}
\hline \multirow{3}{*}{$\begin{array}{r}\text { Burst Responses } \\
\text { Model R } \mathrm{R}^{2}: 0.14\end{array}$} & Group & -0.14 & 0.40 & 11.99 & 0.35 & 0.73 \\
\hline & Session & -0.15 & 0.05 & 11.99 & 3.16 & $<0.01$ \\
\hline & Group*Session & 0.05 & 0.07 & 11.99 & 0.65 & 0.53 \\
\hline \multirow{3}{*}{$\begin{array}{l}\text { Unreinf:reinf ratio } \\
\text { Model } R^{2}: 0.18\end{array}$} & Group & -0.33 & 0.35 & 74.34 & 0.93 & 0.36 \\
\hline & Session & -0.17 & 0.03 & 124 & 5.09 & $<0.01$ \\
\hline & Group*Session & 0.06 & 0.05 & 124 & 1.15 & 0.25 \\
\hline \multirow{3}{*}{$\begin{array}{r}\text { Median IRT } \\
\text { Model R }{ }^{2} \text { : }\end{array}$} & Group & 0.62 & 0.57 & 12 & 1.08 & 0.30 \\
\hline & Session & 0.18 & 0.06 & 12 & 2.87 & 0.04 \\
\hline & Group*Session & -0.07 & 0.10 & 12 & 0.78 & 0.45 \\
\hline \multirow{3}{*}{$\begin{array}{r}\text { Modal IRT } \\
\text { Model } \mathrm{R}^{2} \text { : }\end{array}$} & Group & -0.05 & 0.33 & 99.43 & 0.14 & 0.89 \\
\hline & Session & 0.18 & 0.03 & 124 & 5.27 & $<0.01$ \\
\hline & Group*Session & 0.01 & 0.05 & 124 & 0.1 & 0.92 \\
\hline \multicolumn{7}{|l|}{ DRL 10 - Male } \\
\hline & Group & 0.79 & 0.47 & 13 & 1.66 & 0.12 \\
\hline \multirow{2}{*}{ Model $R^{2}: 0.05$} & Session & -0.04 & 0.04 & 13 & 1.02 & 0.32 \\
\hline & Group*Session & -0.06 & 0.05 & 13 & 1.12 & 0.28 \\
\hline \multirow{3}{*}{$\begin{array}{l}\text { Burst resp (6-15) } \\
\text { Model R } R^{2}: 0.03\end{array}$} & Group & 0.46 & 0.67 & 13 & 0.69 & 0.49 \\
\hline & Session & -0.09 & 0.13 & 13 & 0.07 & 0.99 \\
\hline & Group*Session & -0.05 & 0.19 & 13 & 0.25 & 0.80 \\
\hline \multicolumn{7}{|l|}{ DRL 10 - Female } \\
\hline Burst resp (1-5) & Group & -0.75 & 0.74 & 12 & 1.02 & 0.33 \\
\hline \multirow[t]{2}{*}{ Model $\mathrm{R}^{2}: 0.03$} & Session & -0.04 & 0.04 & 12 & 0.93 & 0.37 \\
\hline & Group*Session & 0.04 & 0.07 & 12 & 0.60 & 0.56 \\
\hline \multirow{3}{*}{$\begin{array}{l}\text { Burst resp (6-15) } \\
\text { Model R } R^{2}: 0.02\end{array}$} & Group & 0.49 & 0.81 & 12 & 0.61 & 0.55 \\
\hline & Session & 0.05 & 0.15 & 12 & 0.34 & 0.74 \\
\hline & Group*Session & -0.16 & 0.23 & 12 & 0.71 & 0.40 \\
\hline \multicolumn{7}{|l|}{ DRL 20 - Male } \\
\hline \multirow{2}{*}{$\begin{array}{r}\text { Burst resp } \\
\text { Model } \mathrm{R}^{2}: 0.05\end{array}$} & Group & -0.10 & 0.23 & 13 & 0.44 & 0.67 \\
\hline & Session & -0.04 & 0.01 & 13 & 6.05 & $<0.01$ \\
\hline
\end{tabular}




\begin{tabular}{|c|c|c|c|c|c|c|}
\hline & Group*Session & 0.02 & 0.01 & 13 & 2.36 & 0.03 \\
\hline $\begin{array}{r}\text { Median IRT } \\
\text { Model R } \mathrm{R}^{2}: 0.19\end{array}$ & $\begin{array}{l}\text { Group } \\
\text { Session } \\
\text { Group*Session }\end{array}$ & $\begin{array}{c}0.06 \\
\mathbf{0 . 1 2} \\
\mathbf{- 0 . 0 6}\end{array}$ & $\begin{array}{l}0.38 \\
\mathbf{0 . 0 2} \\
\mathbf{0 . 0 3}\end{array}$ & $\begin{array}{l}13 \\
13 \\
13\end{array}$ & $\begin{array}{l}0.16 \\
\mathbf{6 . 0 6} \\
\mathbf{2 . 2 3}\end{array}$ & $\begin{array}{c}0.87 \\
<\mathbf{0 . 0 1} \\
\mathbf{0 . 0 4}\end{array}$ \\
\hline $\begin{array}{r}\text { Modal IRT } \\
\text { Model R } R^{2}: 0.29\end{array}$ & $\begin{array}{l}\text { Group } \\
\text { Session } \\
\text { Group*Session }\end{array}$ & $\begin{array}{c}-0.21 \\
\mathbf{0 . 1 3} \\
-0.01\end{array}$ & $\begin{array}{l}0.42 \\
\mathbf{0 . 0 2} \\
0.03\end{array}$ & $\begin{array}{l}13 \\
13 \\
13\end{array}$ & $\begin{array}{l}0.51 \\
\mathbf{5 . 7 0} \\
0.37\end{array}$ & $\begin{array}{c}0.62 \\
<\mathbf{0 . 0 1} \\
0.71\end{array}$ \\
\hline DRL 20 - Female & & & & & & \\
\hline $\begin{array}{r}\text { Burst resp } \\
\text { Model } \mathrm{R}^{2}: 0.03\end{array}$ & $\begin{array}{l}\text { Group } \\
\text { Session } \\
\text { Group*Session }\end{array}$ & $\begin{array}{l}0.39 \\
\mathbf{- 0 . 0 7} \\
-0.03\end{array}$ & $\begin{array}{l}0.59 \\
\mathbf{0 . 0 3} \\
0.05\end{array}$ & $\begin{array}{l}12 \\
12 \\
12\end{array}$ & $\begin{array}{c}0.66 \\
\mathbf{2 . 1} \\
0.67\end{array}$ & $\begin{array}{l}0.52 \\
\mathbf{0 . 0 5} \\
0.51\end{array}$ \\
\hline $\begin{array}{r}\text { Median IRT } \\
\text { Model R } \mathrm{R}^{2}: 0.17\end{array}$ & $\begin{array}{l}\text { Group } \\
\text { Session } \\
\text { Group*Session }\end{array}$ & $\begin{array}{r}-0.37 \\
\mathbf{0 . 0 8} \\
0.04\end{array}$ & $\begin{array}{l}0.46 \\
\mathbf{0 . 0 2} \\
0.03\end{array}$ & $\begin{array}{l}12 \\
12 \\
12\end{array}$ & $\begin{array}{l}0.80 \\
\mathbf{3 . 9 2} \\
1.13\end{array}$ & $\begin{array}{c}0.44 \\
<\mathbf{0 . 0 1} \\
0.28\end{array}$ \\
\hline $\begin{array}{l}\text { Modal IRT } \\
\text { Model R²: }\end{array}$ & $\begin{array}{l}\text { Group } \\
\text { Session } \\
\text { Group*Session }\end{array}$ & $\begin{array}{c}-0.18 \\
\mathbf{0 . 1 4} \\
-0.01\end{array}$ & $\begin{array}{l}0.56 \\
\mathbf{0 . 0 3} \\
0.04\end{array}$ & $\begin{array}{l}12 \\
\mathbf{1 2} \\
12\end{array}$ & $\begin{array}{l}0.32 \\
\mathbf{5 . 1 6} \\
0.25\end{array}$ & $\begin{array}{c}0.76 \\
<\mathbf{0 . 0 1} \\
0.81\end{array}$ \\
\hline \multicolumn{7}{|c|}{ DRL 30 - Male (first 15 sessions) } \\
\hline $\begin{array}{r}\text { Reinforcement rate } \\
\text { Model } \mathrm{R}^{2}: 0.18\end{array}$ & $\begin{array}{l}\text { Group } \\
\text { Session } \\
\text { Group*Session }\end{array}$ & $\begin{array}{c}-0.11 \\
\mathbf{0 . 1 0} \\
-0.06\end{array}$ & $\begin{array}{l}0.56 \\
\mathbf{0 . 0 2} \\
0.03\end{array}$ & $\begin{array}{l}13 \\
13 \\
13\end{array}$ & $\begin{array}{l}0.20 \\
\mathbf{5 . 1 0} \\
2.12\end{array}$ & $\begin{array}{c}0.84 \\
<\mathbf{0 . 0 1} \\
0.056\end{array}$ \\
\hline $\begin{array}{r}\text { Response rate } \\
\text { Model } \mathrm{R}^{2}: 0.16\end{array}$ & $\begin{array}{l}\text { Group } \\
\text { Session } \\
\text { Group*Session }\end{array}$ & $\begin{array}{c}0.25 \\
\mathbf{- 0 . 0 8} \\
0.05\end{array}$ & $\begin{array}{l}0.48 \\
\mathbf{0 . 0 2} \\
0.03\end{array}$ & $\begin{array}{l}13 \\
13 \\
13\end{array}$ & $\begin{array}{l}0.53 \\
\mathbf{3 . 8 0} \\
1.76\end{array}$ & $\begin{array}{c}0.61 \\
<\mathbf{0 . 0 1} \\
0.10\end{array}$ \\
\hline $\begin{array}{l}\text { Burst response } \\
\text { Model } \mathrm{R}^{2}: 0.03\end{array}$ & $\begin{array}{l}\text { Group } \\
\text { Session } \\
\text { Group*Session }\end{array}$ & $\begin{array}{l}0.35 \\
-0.02 \\
-0.05\end{array}$ & $\begin{array}{l}0.49 \\
0.02 \\
0.03\end{array}$ & $\begin{array}{l}13 \\
13 \\
13\end{array}$ & $\begin{array}{l}0.70 \\
1.04 \\
0.18\end{array}$ & $\begin{array}{l}0.49 \\
0.32 \\
0.86\end{array}$ \\
\hline $\begin{array}{r}\text { Unreinf:reinf ratio } \\
\text { Model } \mathrm{R}^{2}: 0.20\end{array}$ & $\begin{array}{l}\text { Group } \\
\text { Session }\end{array}$ & $\begin{array}{r}0.05 \\
\mathbf{- 0 . 1 1}\end{array}$ & $\begin{array}{l}0.49 \\
\mathbf{0 . 0 2}\end{array}$ & $\begin{array}{l}13 \\
13\end{array}$ & $\begin{array}{l}0.11 \\
5.64\end{array}$ & $\begin{array}{c}0.92 \\
<\mathbf{0 . 0 1}\end{array}$ \\
\hline
\end{tabular}




$\begin{array}{rlccccc} & \text { Group*Session } & \mathbf{0 . 0 7} & \mathbf{0 . 0 3} & \mathbf{1 3} & \mathbf{2 . 6 1} & \mathbf{0 . 0 2} \\ \text { Median IRT } & \text { Group } & -0.36 & 0.47 & 13 & 0.76 & 0.46 \\ \text { Model R }{ }^{2}: 0.14 & \text { Session } & \mathbf{0 . 0 6} & \mathbf{0 . 0 2} & \mathbf{1 3} & \mathbf{3 . 2 7} & <\mathbf{0 . 0 1} \\ & \text { Group*Session } & -0.04 & 0.02 & 13 & 1.70 & 0.11 \\ \text { Modal IRT } & \text { Group } & 0.49 & 0.47 & 13 & 1.04 & 0.31 \\ \text { Model R R }: 0.07 & \text { Session } & \mathbf{0 . 0 9} & \mathbf{0 . 0 3} & \mathbf{1 3} & \mathbf{3 . 1 3} & <\mathbf{0 . 0 1} \\ & \text { Group*Session } & -0.06 & 0.04 & 13 & 1.53 & 0.15\end{array}$

DRL 30 - Female (first 15 sessions)

$\begin{array}{cl}\text { Modal IRT } & \text { Group } \\ \text { Model R }{ }^{2}: & \text { Session } \\ & \text { Group*Session }\end{array}$

\section{$-0.27$}

\subsection{9}

0.02

25.43

0.68

0.49

0.50

0.01

0.03

194

0.48

0.62

194

0.63

DRL 30 - Male (all sessions)

$\begin{aligned} \text { Burst response } & \text { Group } \\ \text { Model R } R^{2}: 0.03 & \text { Session } \\ & \text { Group*Session } \\ \text { Median IRT } & \text { Group } \\ \text { Model R }{ }^{2}: 0.17 & \text { Session } \\ & \text { Group*Session } \\ \text { Modal IRT } & \text { Group } \\ \text { Model R } \text { R }^{2}: 0.12 & \text { Session } \\ & \text { Group*Session }\end{aligned}$

\subsection{9} $-0.07$

$-0.04$

\subsection{6}

0.01

13

0.69

$-0.60$

0.01

13

13

$\begin{array}{cc}0.69 & 0.50 \\ 0.67 & 0.52 \\ 0.26 & 0.80 \\ 1.38 & 0.19 \\ \mathbf{3 . 7 5} & <\mathbf{0 . 0 1} \\ 0.77 & 0.45 \\ 1.04 & 0.32 \\ \mathbf{3 . 1 3} & <\mathbf{0 . 0 1} \\ 1.53 & 0.15\end{array}$

DRL 30 - Female (all sessions)

\begin{tabular}{cl}
\hline Modal IRT & Group \\
Model R ${ }^{2}: 0.03$ & Session \\
& Group*Session \\
\hline
\end{tabular}

\section{$\mathbf{0 . 0 3}$}

0.44

0.08

0.01

13

13

$-0.09$

0.47

0.09

$\mathbf{0 . 0 3}$

$-0.06$

0.04

\section{$-0.30$}

0.44

$0.01 \quad 0.01$

0.01

$\begin{array}{ll}12 & 0.69 \\ 12 & 1.34 \\ 12 & 0.96\end{array}$

0.01

\footnotetext{
Note. Bolded values indicate statistical significance $(p<0.05)$.
} 
Table 5. Nonlinear regression outcomes for Experiment 3

\begin{tabular}{|c|c|c|c|c|c|c|}
\hline DRL 10 - Male & Effect & $b$ & Std. Error & $d f$ & $t$ & $p$ \\
\hline \multirow[t]{2}{*}{ Reinforcement Rate } & Group (int) & 0.39 & 0.26 & 207 & 1.52 & 0.13 \\
\hline & Group (slope) & -0.11 & 0.07 & 207 & 1.67 & 0.09 \\
\hline Response Rate & Group (slope) & -0.06 & 0.09 & 209 & 0.63 & 0.53 \\
\hline Unreinf:reinf ratio & Group (slope) & 0.06 & 0.09 & 209 & 0.74 & 0.46 \\
\hline \multirow[t]{2}{*}{ Median IRT } & Group (int) & 0.15 & 0.81 & 207 & 0.19 & 0.85 \\
\hline & Group (slope) & -0.06 & 0.11 & 207 & 0.62 & 0.54 \\
\hline Modal IRT & Group (slope) & 0.07 & 0.03 & 209 & 2.33 & 0.02 \\
\hline \multicolumn{7}{|l|}{ DRL 10 - Female } \\
\hline \multirow[t]{2}{*}{ Reinforcement Rate } & Group (int) & 0.01 & 0.20 & 193 & 0.07 & 0.94 \\
\hline & Group (slope) & -0.03 & 0.04 & 193 & 0.64 & 0.25 \\
\hline Response Rate & Group (slope) & 0.07 & 0.08 & 195 & 0.95 & 0.34 \\
\hline Unreinf:reinf ratio & Group (slope) & 0.01 & 0.08 & 195 & 0.16 & 0.87 \\
\hline \multirow[t]{2}{*}{ Median IRT } & Group (int) & -0.07 & 0.75 & 193 & 0.09 & 0.92 \\
\hline & Group (slope) & 0.04 & 0.07 & 193 & 0.56 & 0.57 \\
\hline Modal IRT & Group (slope) & 0.03 & 0.02 & 195 & 1.36 & 0.17 \\
\hline \multicolumn{7}{|l|}{ DRL 20 - Male } \\
\hline \multirow[t]{2}{*}{ Reinforcement Rate } & Group (int) & 0.01 & 0.14 & 207 & 0.09 & 0.93 \\
\hline & Group (slope) & -0.04 & 0.12 & 207 & 0.37 & 0.71 \\
\hline Response Rate & Group (slope) & -0.01 & 0.02 & 209 & 0.46 & 0.65 \\
\hline \multirow[t]{2}{*}{ Unreinf:reinf ratio } & Group (slope) & -0.15 & 0.14 & 405 & 1.12 & 0.27 \\
\hline & & & & \multicolumn{3}{|c|}{ (continued on next page } \\
\hline
\end{tabular}




\begin{tabular}{|c|c|c|c|c|c|c|}
\hline \multicolumn{7}{|l|}{ DRL 20 - Female } \\
\hline \multirow[t]{2}{*}{$\overline{\text { Reinforcement Rate }}$} & Group (int) & -0.19 & 0.16 & 193 & 1.19 & 0.23 \\
\hline & Group (slope) & 0.04 & 0.25 & 193 & 0.16 & 0.87 \\
\hline Response Rate & Group (slope) & -0.02 & 0.02 & 195 & 0.94 & 0.34 \\
\hline Unreinf:reinf ratio & Group (slope) & -0.11 & 0.17 & 195 & 0.62 & 0.53 \\
\hline \multicolumn{7}{|c|}{ DRL 30 - Female (first 15 sessions) } \\
\hline \multirow[t]{2}{*}{ Reinforcement Rate } & Group (int) & -0.18 & 0.14 & 193 & 1.24 & 0.22 \\
\hline & Group (slope) & 0.12 & 0.07 & 193 & 1.61 & 0.11 \\
\hline Response Rate & Group (slope) & -0.01 & 0.02 & 195 & 0.81 & 0.42 \\
\hline Burst resp & Group (slope) & -0.08 & 0.07 & 195 & 1.11 & 0.27 \\
\hline \multirow[t]{2}{*}{ Unreinf:reinf ratio } & Group (int) & -5.80 & 3.91 & 193 & 1.48 & 0.14 \\
\hline & Group (slope) & -0.66 & 0.24 & 193 & 2.75 & $<0.01$ \\
\hline Median IRT & Group (slope) & -0.06 & 0.06 & 195 & 0.09 & 0.93 \\
\hline \multicolumn{7}{|c|}{ DRL 30 - Male (all sessions) } \\
\hline \multirow[t]{2}{*}{ Reinforcement Rate } & Group (int) & -0.04 & 0.16 & 432 & 0.26 & 0.79 \\
\hline & Group (slope) & 0.17 & 0.16 & 432 & 1.11 & 0.27 \\
\hline Response Rate & Group (slope) & -0.01 & $\mathbf{0 . 0 7}$ & 434 & 2.12 & $\mathbf{0 . 0 3}$ \\
\hline Unreinf:reinf ratio & Group (slope) & -0.15 & 0.14 & 434 & 1.06 & 0.29 \\
\hline \multicolumn{7}{|c|}{ DRL 30 - Female (all sessions) } \\
\hline \multirow[t]{2}{*}{ Reinforcement Rate } & Group (int) & -0.13 & 0.14 & 403 & 0.93 & 0.35 \\
\hline & Group (slope) & 0.07 & 0.06 & 403 & 1.18 & 0.24 \\
\hline Response Rate & Group (slope) & -0.05 & 0.09 & 405 & 0.56 & 0.57 \\
\hline Burst resp & Group (slope) & -0.06 & 0.06 & 405 & 1.05 & 0.29 \\
\hline Unreinf:reinf ratio & Group (slope) & -0.73 & 0.55 & 405 & 1.35 & 0.18 \\
\hline Median IRT & Group (slope) & 0.01 & 0.04 & 405 & 0.02 & 0.98 \\
\hline
\end{tabular}

Note. Bolded values indicate statistical significance $(p<0.05)$. 
Table 6. ANOVA outcomes for DRL transitions of Experiment 3 - Males only

\begin{tabular}{|c|c|c|c|c|c|}
\hline Transition \& measure & Effect & $F$ & $d f$ & $p$ & $\eta_{\mathrm{p}}{ }^{2}$ \\
\hline \multicolumn{6}{|l|}{ DRL 5 - DRL 10} \\
\hline \multirow[t]{3}{*}{ Reinforcement Rate } & Group & 0.34 & 1,13 & 0.57 & 0.03 \\
\hline & DRL & 720.12 & 1,13 & $<0.01$ & 0.98 \\
\hline & Group*DRL & 1.37 & 1,13 & 0.26 & 0.09 \\
\hline \multirow[t]{3}{*}{ Response Rate } & Group & 0.02 & 1,13 & 0.88 & $<0.01$ \\
\hline & DRL & 39.68 & 1,13 & $<0.01$ & 0.75 \\
\hline & Group*DRL & $<0.01$ & 1,13 & 0.97 & $<0.01$ \\
\hline \multirow[t]{3}{*}{ Burst Response } & Group & 0.43 & 1,13 & 0.52 & 0.03 \\
\hline & DRL & 23.97 & 1,13 & $<0.01$ & 0.65 \\
\hline & Group*DRL & 0.24 & 1,13 & 0.63 & 0.02 \\
\hline \multirow[t]{3}{*}{ Unreinf:reinf Ratio } & Group & 1.15 & 1,13 & 0.30 & 0.08 \\
\hline & DRL & 47.10 & 1,13 & 0.25 & 0.78 \\
\hline & Group*DRL & 1.45 & 1,13 & 0.25 & 0.10 \\
\hline \multirow[t]{3}{*}{ Median IRT } & Group & $<0.01$ & 1,13 & 0.93 & $<0.01$ \\
\hline & DRL & 14.72 & 1,13 & $<0.01$ & 0.53 \\
\hline & Group*DRL & $<0.01$ & 1,13 & 0.97 & $<0.01$ \\
\hline \multirow[t]{3}{*}{ Modal IRT } & Group & 0.13 & 1,13 & 0.72 & 0.01 \\
\hline & DRL & 2.89 & 1,13 & 0.11 & 0.18 \\
\hline & Group*DRL & 0.34 & 1,13 & 0.57 & 0.02 \\
\hline \multicolumn{6}{|l|}{ DRL 10 - DRL 20} \\
\hline \multirow{3}{*}{ Reinforcement Rate } & Group & 1.47 & 1,13 & 0.25 & 0.10 \\
\hline & DRL & 754.28 & 1,13 & $<0.01$ & 0.98 \\
\hline & Group*DRL & 2.62 & 1,13 & 0.13 & 0.17 \\
\hline \multirow[t]{3}{*}{ Response Rate } & Group & $<0.01$ & 1,13 & 0.99 & $<0.01$ \\
\hline & DRL & 0.42 & 1,13 & 0.53 & 0.03 \\
\hline & Group*DRL & 0.31 & 1,13 & 0.59 & 0.02 \\
\hline \multirow{3}{*}{ Burst Response } & Group & 0.04 & 1,13 & 0.83 & $<0.01$ \\
\hline & DRL & 22.26 & 1,13 & $<0.01$ & 0.63 \\
\hline & Group*DRL & 0.36 & 1,13 & 0.56 & 0.02 \\
\hline \multirow[t]{3}{*}{ Unreinf:reinf Ratio } & Group & 0.51 & 1,13 & 0.48 & 0.04 \\
\hline & DRL & 33.88 & 1,13 & $<0.01$ & 0.72 \\
\hline & Group*DRL & 0.59 & 1,13 & 0.45 & 0.04 \\
\hline \multirow[t]{3}{*}{ Median IRT } & Group & $<0.01$ & 1,13 & 0.93 & $<0.01$ \\
\hline & DRL & 0.78 & 1,13 & 0.39 & 0.05 \\
\hline & Group*DRL & 0.25 & 1,13 & 0.62 & 0.02 \\
\hline \multirow[t]{2}{*}{ Modal IRT } & Group & 1.48 & 1,13 & 0.25 & 0.10 \\
\hline & DRL & 2.77 & 1,13 & 0.12 & 0.17 \\
\hline
\end{tabular}




\begin{tabular}{rrrrrc} 
& Group*DRL & $\mathbf{5 . 6 2}$ & $\mathbf{1 , 1 3}$ & $\mathbf{0 . 0 3}$ & $\mathbf{0 . 3 0}$ \\
DRL 20 - DRL 30 & & & & & \\
\cline { 1 - 2 } Reinforcement Rate & Group & 0.66 & 1,13 & 0.43 & 0.05 \\
& DRL & $\mathbf{1 2 9 . 4 0}$ & $\mathbf{1 , 1 3}$ & $<\mathbf{0 . 0 1}$ & $\mathbf{0 . 9 1}$ \\
Response Rate & Group*DRL & 1.78 & 1,13 & 0.21 & 0.12 \\
& Group & 2.39 & 1,13 & 0.15 & 0.15 \\
& DRL & $<0.01$ & 1,13 & 0.95 & $<0.01$ \\
Burst Response & Group*DRL & 0.02 & 1,13 & 0.88 & $<0.01$ \\
& Group & 1.56 & 1,13 & 0.23 & 0.11 \\
& DRL & 4.16 & 1,13 & 0.06 & 0.24 \\
Unreinf:reinf Ratio & Group*DRL & 0.34 & 1,13 & 0.57 & 0.02 \\
& Group & 0.98 & 1,13 & 0.34 & 0.07 \\
& DRL & $\mathbf{3 1 . 9 3}$ & $\mathbf{1 , 1 3}$ & $<\mathbf{0 . 0 1}$ & $\mathbf{0 . 7 1}$ \\
Median IRT & Group*DRL & 0.41 & 1,13 & 0.53 & 0.03 \\
& Group & 2.30 & 1,13 & 0.15 & 0.15 \\
& DRL & 0.58 & 1,13 & 0.46 & 0.04 \\
& Group*DRL & $<0.01$ & 1,13 & 0.95 & $<0.01$ \\
Modal IRT & Group & 0.16 & 1,13 & 0.69 & 0.01 \\
& DRL & 0.11 & 1,13 & 0.74 & $<0.01$ \\
& Group*DRL & 0.16 & 1,13 & 0.69 & 0.01 \\
\hline
\end{tabular}

Note. Bolded values indicate statistical significance $(p<0.05)$. 
Table 7. ANOVA outcomes for DRL transitions of Experiment 3 - Females only

\begin{tabular}{|c|c|c|c|c|c|}
\hline Transition \& measure & Effect & $F$ & $d f$ & $p$ & $\eta_{\mathrm{p}}^{2}$ \\
\hline DRL $5-$ DRL 10 & & & & & \\
\hline \multirow[t]{3}{*}{ Reinforcement Rate } & Group & 0.64 & 1,13 & 0.44 & 0.05 \\
\hline & DRL & 43.19 & $\mathbf{1 , 1 3}$ & $<0.01$ & 0.97 \\
\hline & Group*DRL & 0.13 & 1,13 & 0.72 & 0.01 \\
\hline \multirow[t]{3}{*}{ Response Rate } & Group & 0.52 & 1,13 & 0.48 & 0.04 \\
\hline & DRL & 0.52 & 1,13 & 0.48 & 0.04 \\
\hline & Group*DRL & 1.56 & 1,13 & 0.23 & 0.11 \\
\hline \multirow[t]{3}{*}{ Burst Response } & Group & 1.14 & 1,13 & 0.31 & 0.08 \\
\hline & DRL & 18.87 & 1,13 & $<0.01$ & 0.61 \\
\hline & Group*DRL & 0.54 & 1,13 & 0.48 & 0.04 \\
\hline \multirow[t]{3}{*}{ Unreinf:reinf Ratio } & Group & $<0.01$ & 1,13 & 0.96 & $<0.01$ \\
\hline & DRL & 25.28 & 1,13 & $<0.01$ & 0.68 \\
\hline & Group*DRL & $<0.01$ & 1,13 & 0.93 & $<0.01$ \\
\hline \multirow[t]{3}{*}{ Median IRT } & Group & $<0.01$ & 1,13 & 0.96 & $<0.01$ \\
\hline & DRL & 1.75 & 1,13 & 0.21 & 0.13 \\
\hline & Group*DRL & $<0.01$ & 1,13 & 0.95 & $<0.01$ \\
\hline \multirow[t]{3}{*}{ Modal IRT } & Group & 6.25 & 1,13 & 0.03 & 0.35 \\
\hline & DRL & 17.86 & 1,13 & $<0.01$ & 0.59 \\
\hline & Group*DRL & 4.90 & 1,13 & 0.05 & 0.29 \\
\hline \multicolumn{6}{|l|}{ DRL $10-$ DRL 20} \\
\hline \multirow[t]{3}{*}{ Reinforcement Rate } & Group & 0.49 & 1,13 & 0.49 & 0.04 \\
\hline & DRL & 356.59 & 1,13 & $<0.01$ & 0.97 \\
\hline & Group*DRL & 0.14 & 1,13 & 0.72 & 0.01 \\
\hline \multirow[t]{3}{*}{ Response Rate } & Group & $<0.01$ & 1,13 & 0.99 & $<0.01$ \\
\hline & DRL & $<0.01$ & 1,13 & 0.94 & $<0.01$ \\
\hline & Group*DRL & 0.29 & 1,13 & 0.60 & 0.02 \\
\hline \multirow[t]{3}{*}{ Burst Response } & Group & 0.27 & 1,13 & 0.61 & 0.02 \\
\hline & DRL & 45.80 & 1,13 & $<0.01$ & 0.79 \\
\hline & Group*DRL & 0.68 & 1,13 & 0.43 & 0.05 \\
\hline \multirow[t]{3}{*}{ Unreinf:reinf Ratio } & Group & 0.37 & 1,13 & 0.56 & 0.03 \\
\hline & DRL & 36.56 & 1,13 & $<0.01$ & 0.75 \\
\hline & Group*DRL & 0.38 & 1,13 & 0.55 & 0.03 \\
\hline \multirow[t]{3}{*}{ Median IRT } & Group & 0.14 & 1,13 & 0.71 & 0.01 \\
\hline & DRL & 5.18 & $\mathbf{1 , 1 3}$ & 0.04 & 0.30 \\
\hline & Group*DRL & 0.72 & 1,13 & 0.41 & 0.06 \\
\hline \multirow[t]{3}{*}{ Modal IRT } & Group & 0.04 & 1,13 & 0.85 & $<0.01$ \\
\hline & DRL & 1.58 & 1,13 & 0.23 & 0.12 \\
\hline & & & \multicolumn{3}{|c|}{ (continued on next page) } \\
\hline
\end{tabular}




\begin{tabular}{rrrccc} 
& Group*DRL & 0.03 & 1,13 & 0.85 & $<0.01$ \\
DRL 20-DRL 30 & & & & & \\
\cline { 1 - 2 } Reinforcement Rate & Group & 1.32 & 1,13 & 0.27 & 0.09 \\
& DRL & $\mathbf{1 3 8 . 8 3}$ & $\mathbf{1 , 1 3}$ & $<\mathbf{0 . 0 1}$ & $\mathbf{0 . 9 2}$ \\
& Group*DRL & 0.23 & 1,13 & 0.64 & 0.02 \\
Response Rate & Group & 0.25 & 1,13 & 0.62 & 0.02 \\
& DRL & 1.35 & 1,13 & 0.27 & 0.10 \\
& Group*DRL & 0.42 & 1,13 & 0.53 & 0.03 \\
Burst Response & Group & 0.06 & 1,13 & 0.80 & $<0.01$ \\
& DRL & $\mathbf{1 0 . 8 5}$ & $\mathbf{1 , 1 3}$ & $<\mathbf{0 . 0 1}$ & $\mathbf{0 . 4 7}$ \\
& Group*DRL & 0.15 & 1,13 & 0.70 & 0.01 \\
Unreinf:reinf Ratio & Group & 4.37 & 1,13 & 0.06 & 0.27 \\
& DRL & $\mathbf{3 6 . 5 6}$ & $\mathbf{1 , 1 3}$ & $<\mathbf{0 . 0 1}$ & $\mathbf{0 . 4 7}$ \\
& Group*DRL & 3.87 & 1,13 & 0.07 & 0.24 \\
Median IRT & Group & 0.03 & 1,13 & 0.86 & $<0.01$ \\
& DRL & $\mathbf{5 . 3 3}$ & $\mathbf{1 , 1 3}$ & $\mathbf{0 . 0 4}$ & $\mathbf{0 . 3 1}$ \\
& Group*DRL & 0.32 & 1,13 & 0.58 & 0.03 \\
Modal IRT & Group & 1.06 & 1,13 & 0.32 & 0.08 \\
& & & & & \\
& DRL & 2.41 & 1,13 & 0.15 & 0.17 \\
& Group*DRL & 0.10 & 1,13 & 0.76 & $<0.01$ \\
\hline
\end{tabular}

Note. Bolded values indicate statistical significance $(p<0.05)$. 

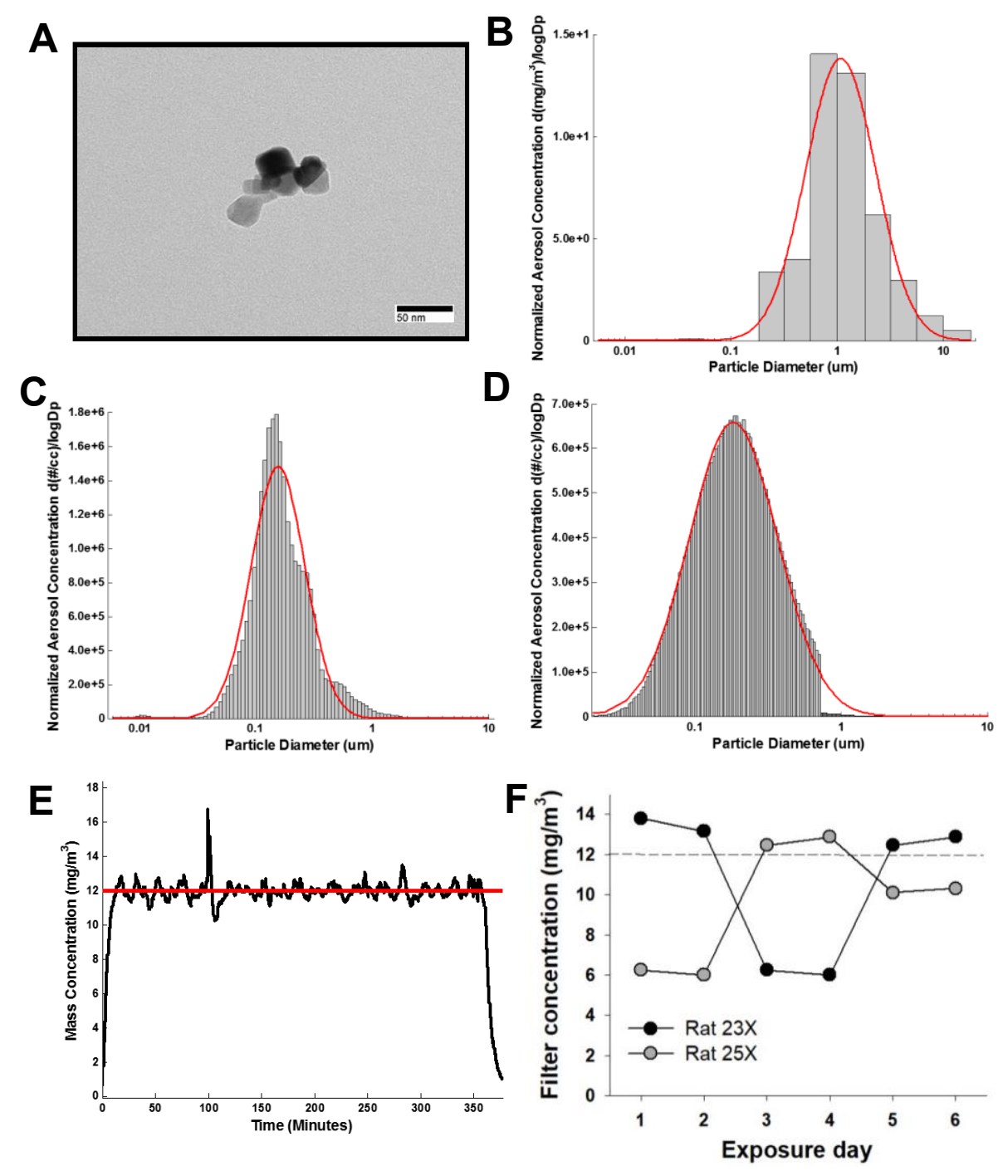

Figure 1. Nano- $\mathrm{TiO}_{2}$ aerosol characterization. (A) Transmission electron microscope image of typical nano- $\mathrm{TiO}_{2}$ agglomerate aerosolized by the acoustical generator. (B) Size distribution of nano- $\mathrm{TiO}_{2}$ aerosol (aerodynamic diameter) sampled from the exposure chamber using a microorifice uniform deposit impactor (MOUDI; count median diameter $=1.08 \mu \mathrm{m})$. (C) Size distribution of nano- $\mathrm{TiO}_{2}$ aerosol using a high resolution electronic low-pressure impactor (ELPI+; count median diameter $=156 \mathrm{~nm}$ ). (D) Size distribution of nano- $\mathrm{TiO}_{2}$ aerosol using a scanning mobility particle sizer (SMPS; light grey bars) and aerodynamic particle sizer (APS; dark grey low values; count median diameter $=184 \mathrm{~nm})$. $(\mathbf{E})$ Real-time mass concentration of nano- $\mathrm{TiO}_{2}$ aerosols during a typical inhalation exposure. (F) Mass concentrations of $\mathrm{TiO}_{2}$ aerosols across exposure days for both pregnant rats that produced $\mathrm{TiO}_{2}$-exposed pups for behavioral assessment. 

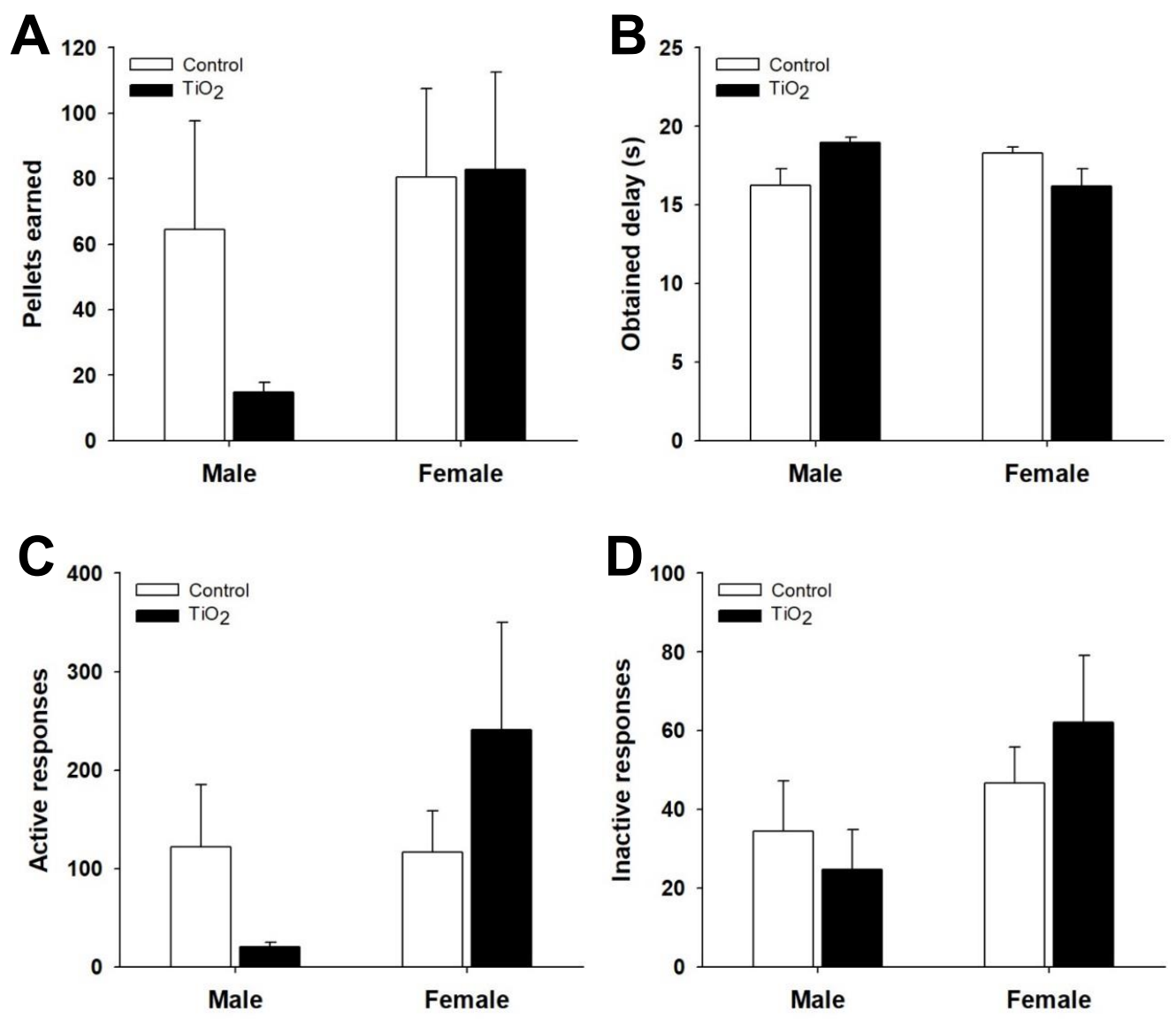

Figure 2. Mean (+ SEM) pellets earned, obtained delay (s), active responses, and inactive responses for both male and female rats during the 8-h acquisition session. Unfilled bars represent air-exposed control rats, and filled bars represent nano- $\mathrm{TiO}_{2}-$ exposed rats. $($ Female $n=8$; Male $n=7-8)$. 
A
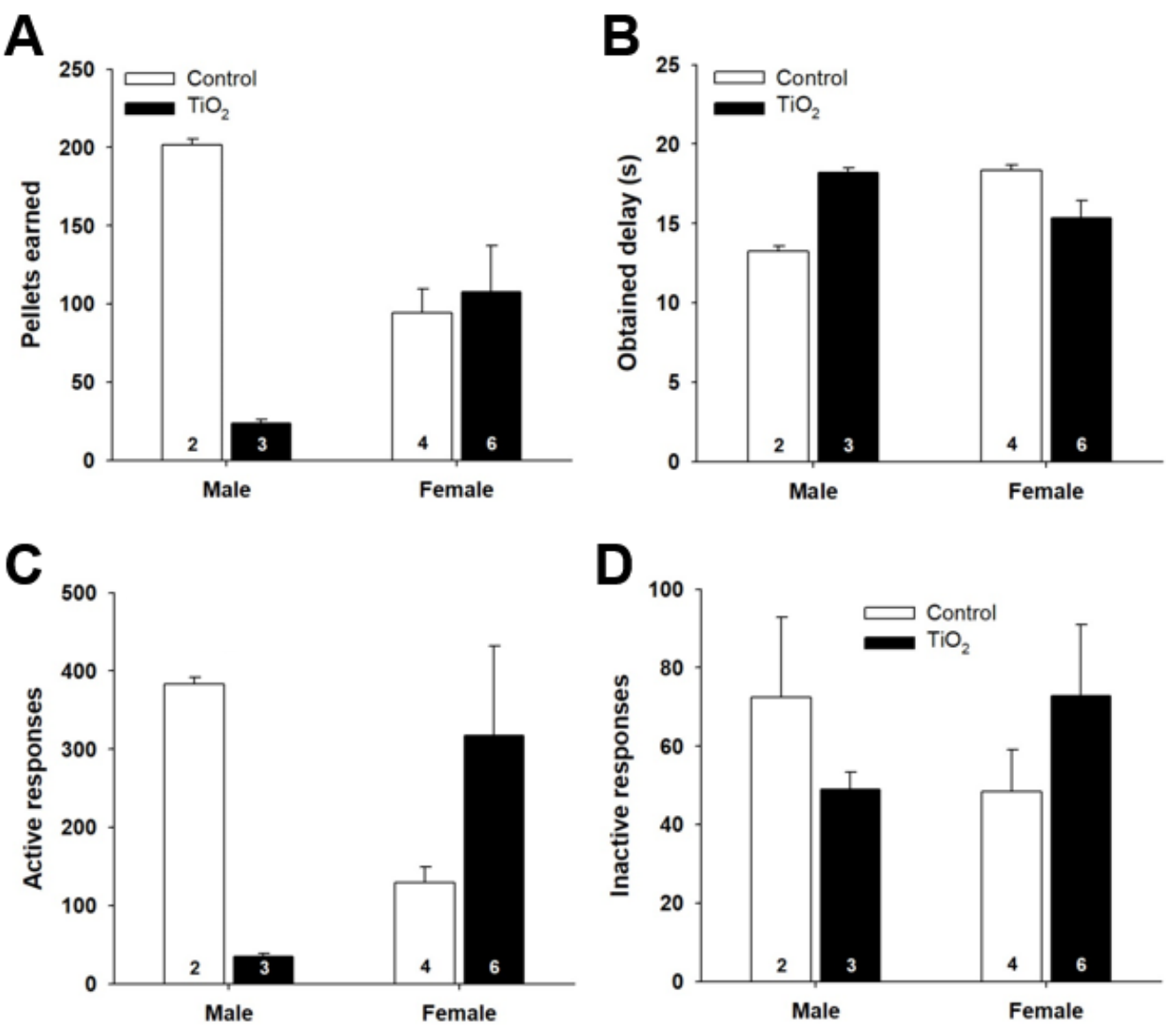

Figure 3. Mean (+ SEM) pellets earned, obtained delay (s), active responses, and inactive responses only for rats that earned $>20$ pellets during the 8 -h acquisition session. Unfilled bars represent air-exposed control, and filled bars represent nano- $\mathrm{TiO}_{2}$-exposed. The numbers at the base of each bar denote the number of rats represented. 


\section{Session 1}
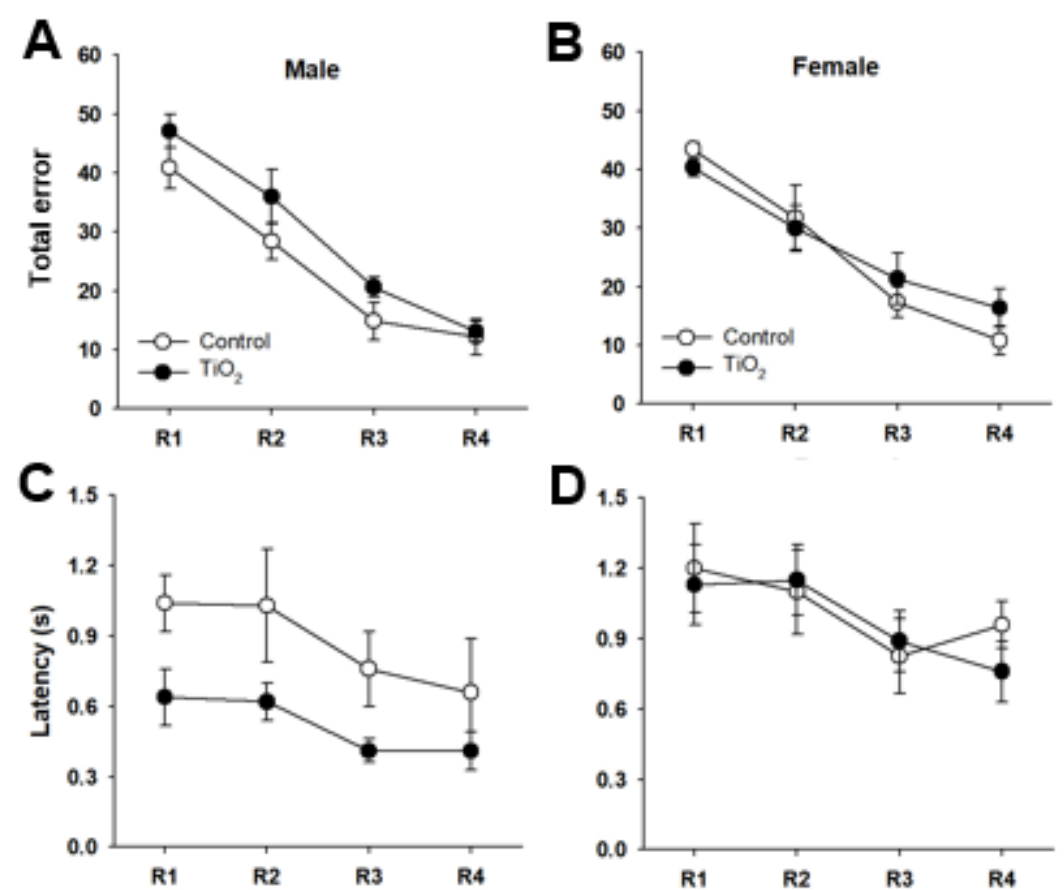

D
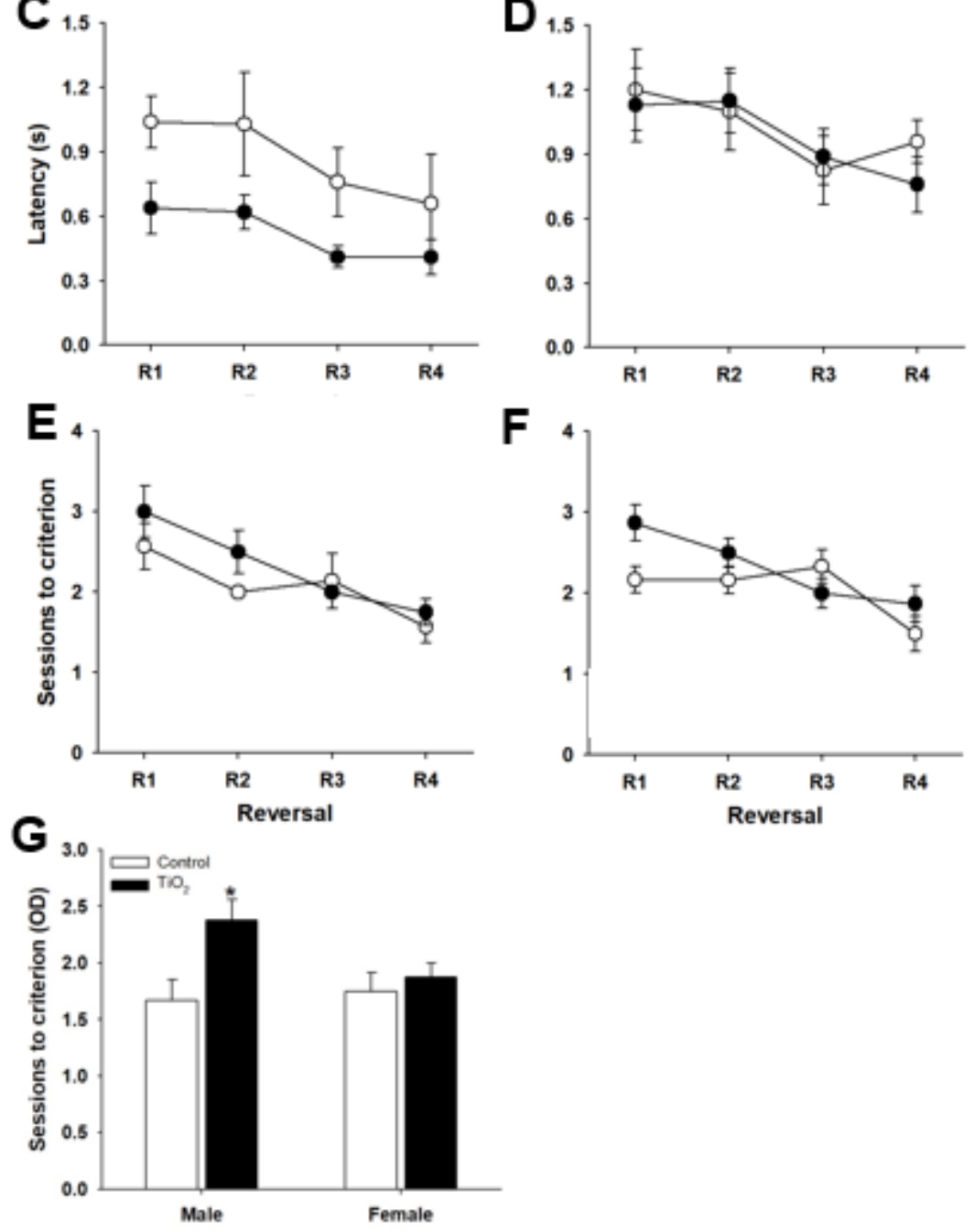

Figure 4. Mean $( \pm \mathrm{SEM})$ error, latency, and sessions to criterion for male and female rats across reversals during Session 1 of each reversal. Panel G only represents sessions to criterion during the original discrimination (OD). Unfilled points/bars represent airexposed control rats, and filled points/bars represent nano- $\mathrm{TiO}_{2}$-exposed rats. (Female $\mathrm{n}=6-8$; Male $\mathrm{n}=7-8)$. ${ }^{*}$ denotes $p<0.05$ vs. Control Male. 


\section{Session 1}
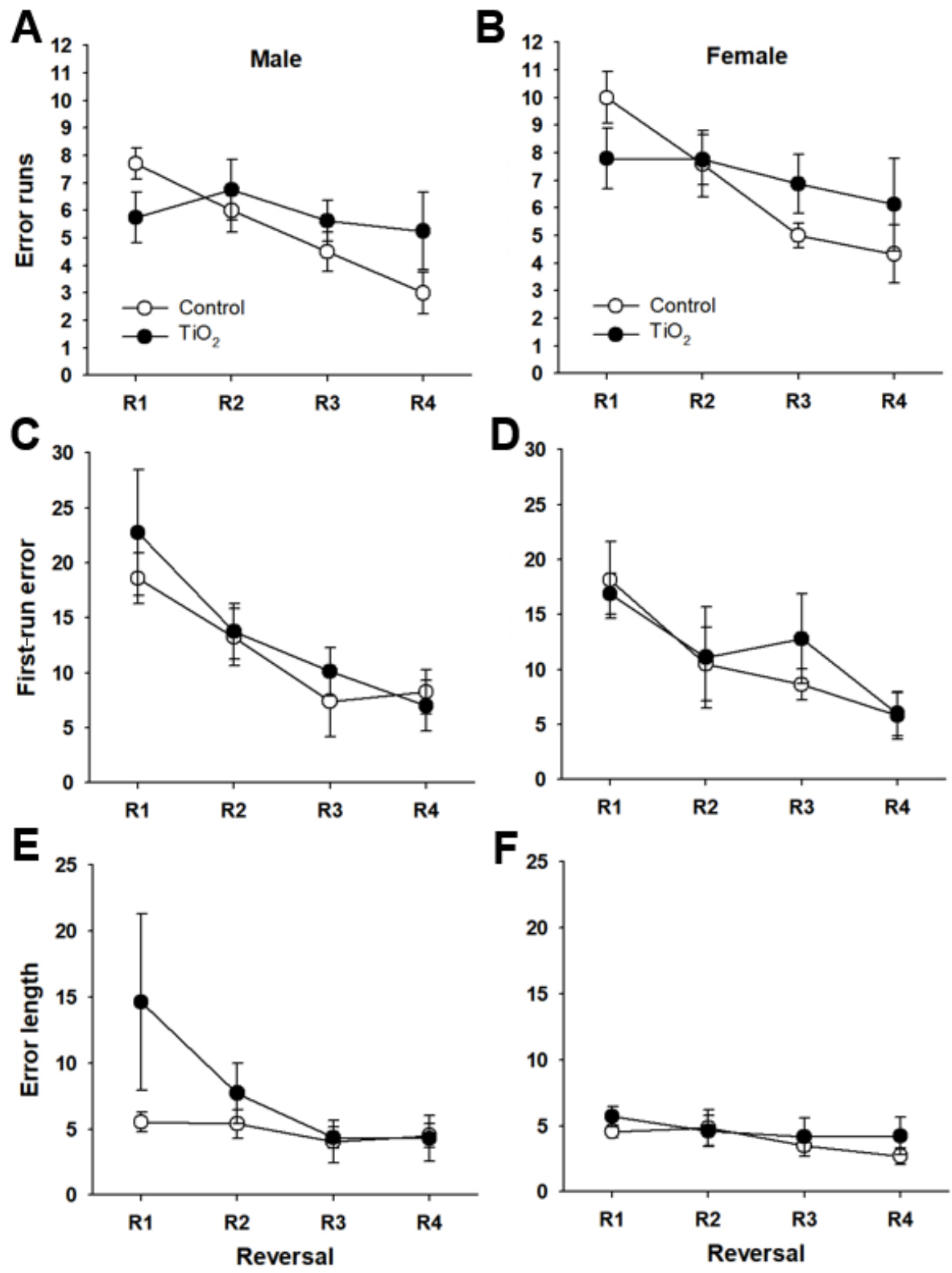

Figure 5. Mean $( \pm \mathrm{SEM})$ error runs, first-run error, and error run length for male and female rats across reversals during Session 1 of each reversal. Unfilled points represent air-exposed control rats, and filled points represent nano- $\mathrm{TiO}_{2}$-exposed rats.

(Female $\mathrm{n}=6-8 ;$ Male $\mathrm{n}=7-8$ ). 


\section{Session 1}

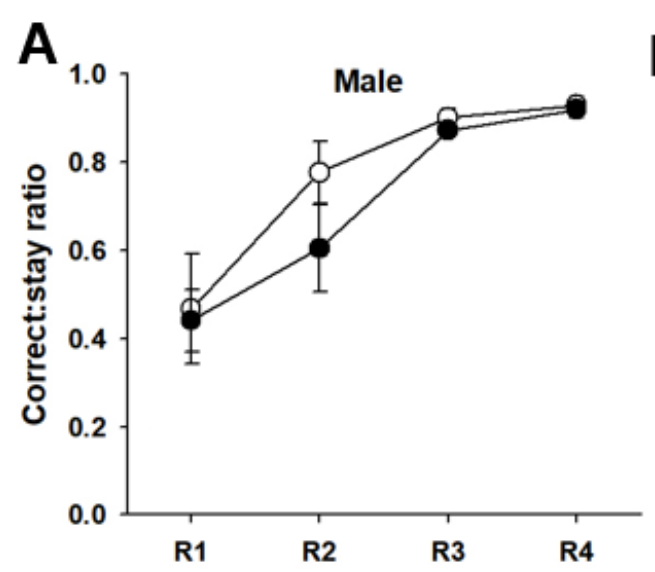

$\mathbf{B}_{1.0}$
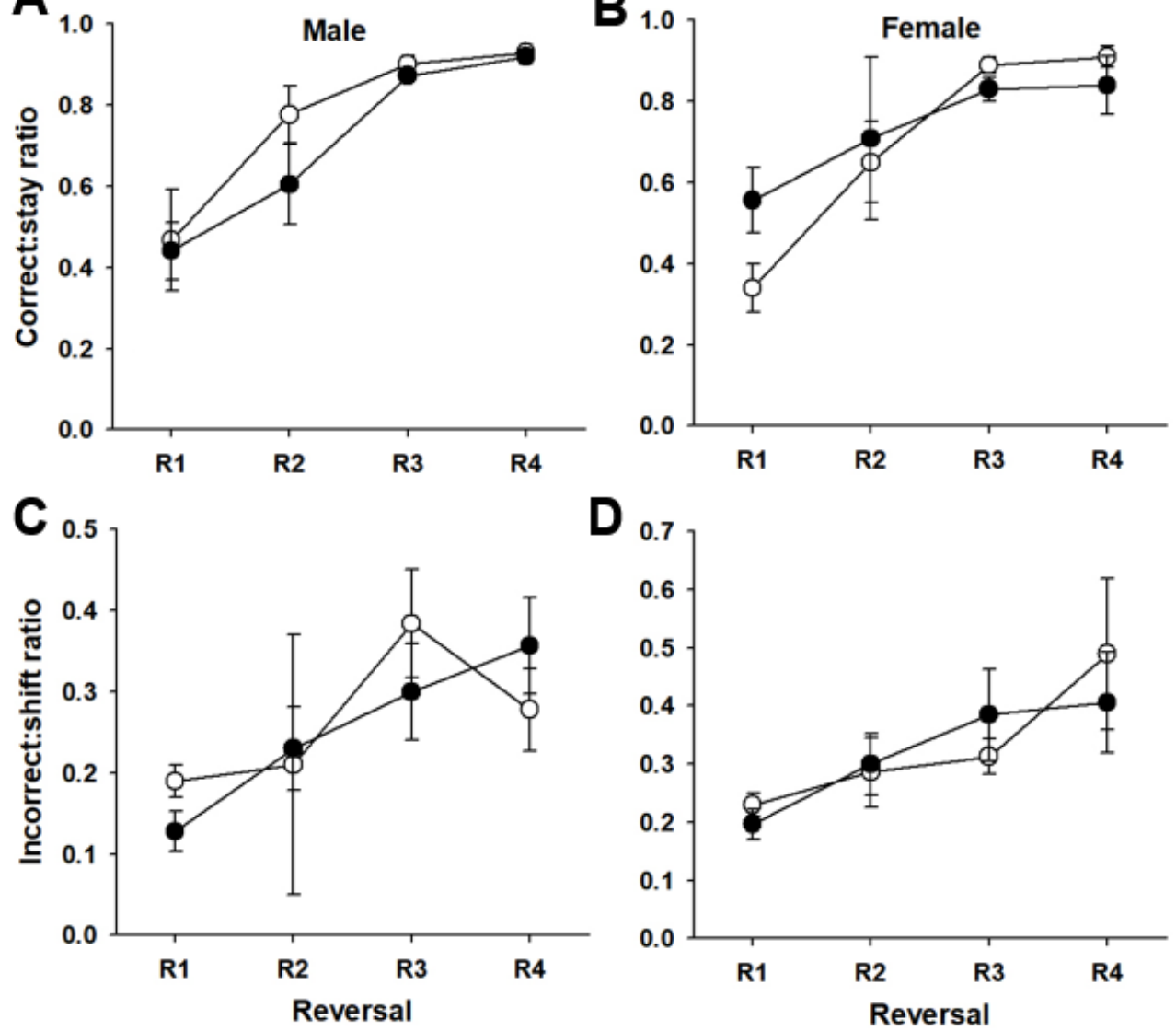

Figure 6. Mean ( $( \pm$ SEM) correct:stay and incorrect:shift ratios for male and female rats across reversals during session 1 of each reversal. Unfilled points represent air-exposed control rats, and filled points represent nano- $\mathrm{TiO}_{2}$-exposed rats.

(Female $\mathrm{n}=6-8$; Male $\mathrm{n}=7-8$ ). 


\section{Session 2}
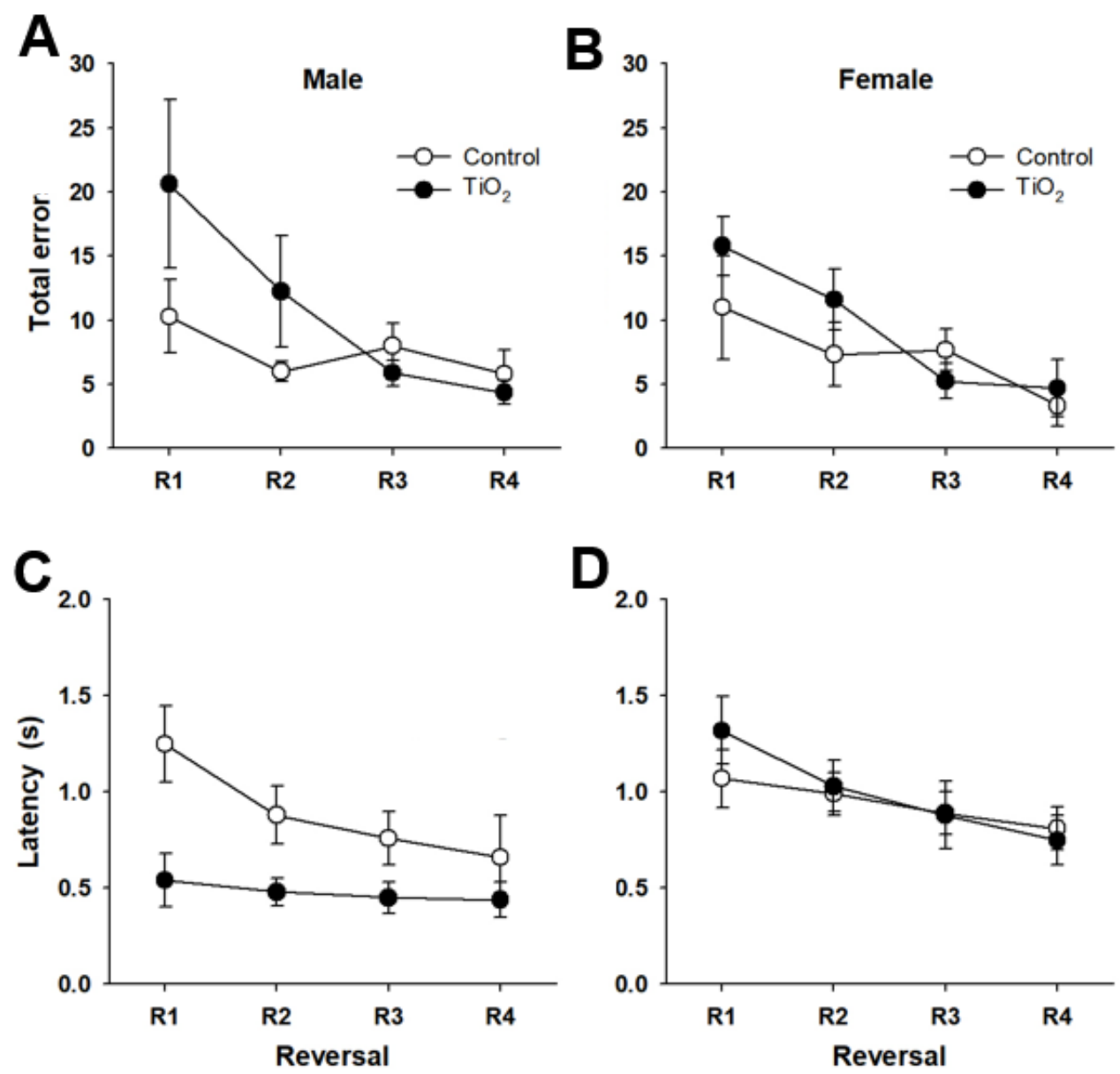

Figure 7. Mean ( $($ SEM) errors and latencies (s) for male and female rats across reversals during Session 2 of each reversal. Unfilled points represent air-exposed control rats, and filled points represent nano- $\mathrm{TiO}_{2}$-exposed rats. (Female $\mathrm{n}=6-8$; Male $\mathrm{n}=7-8$ ). 


\section{Session 2}
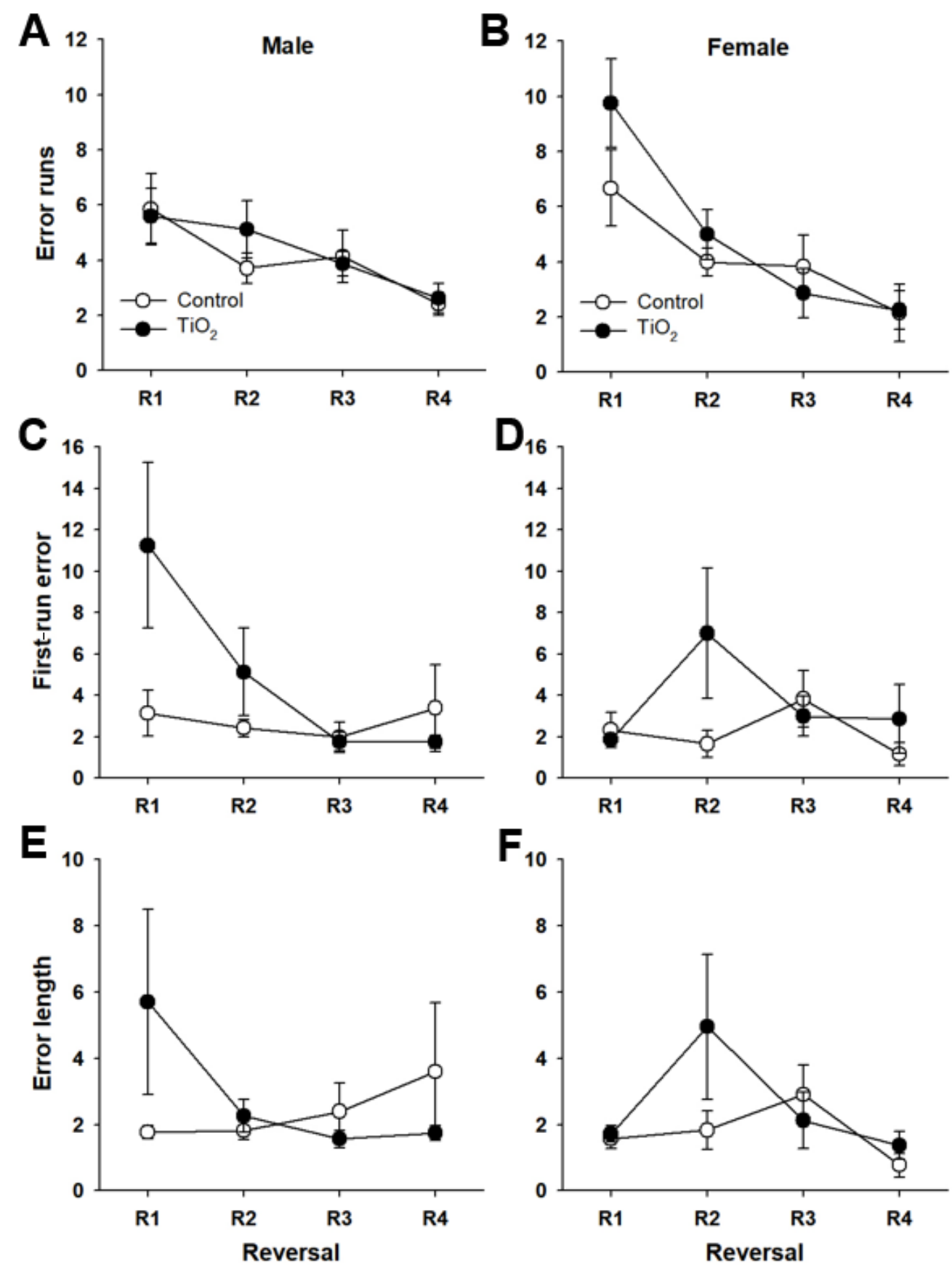

Figure 8. Mean $( \pm$ SEM) error runs, first-run error, and error run length for male and female rats across reversals during Session 2 of each reversal. Unfilled points represent air-exposed control rats, and filled points represent nano- $\mathrm{TiO}_{2}$-exposed rats.

(Female $\mathrm{n}=6-8$; Male $\mathrm{n}=7-8$ ). 


\section{Session 2}
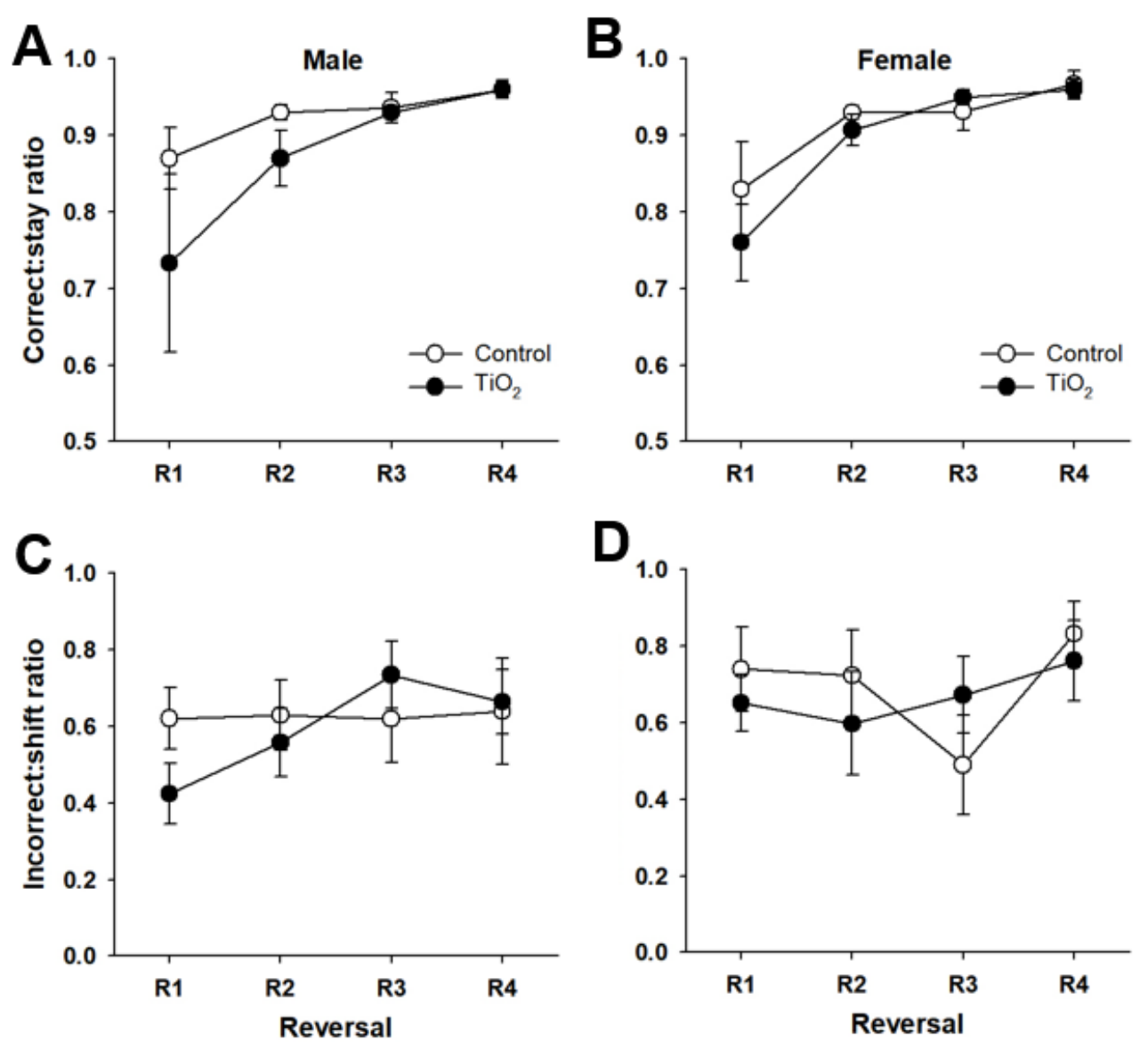

Figure 9. Mean ( \pm SEM) correct:stay and incorrect:shift ratios for male and female rats across reversals during Session 2 of each reversal. Unfilled points represent air-exposed control rats, and filled points represent nano- $\mathrm{TiO}_{2}$-exposed rats.

$($ Female $n=6-8$; Male $n=7-8)$. 


\section{Reversal 1}
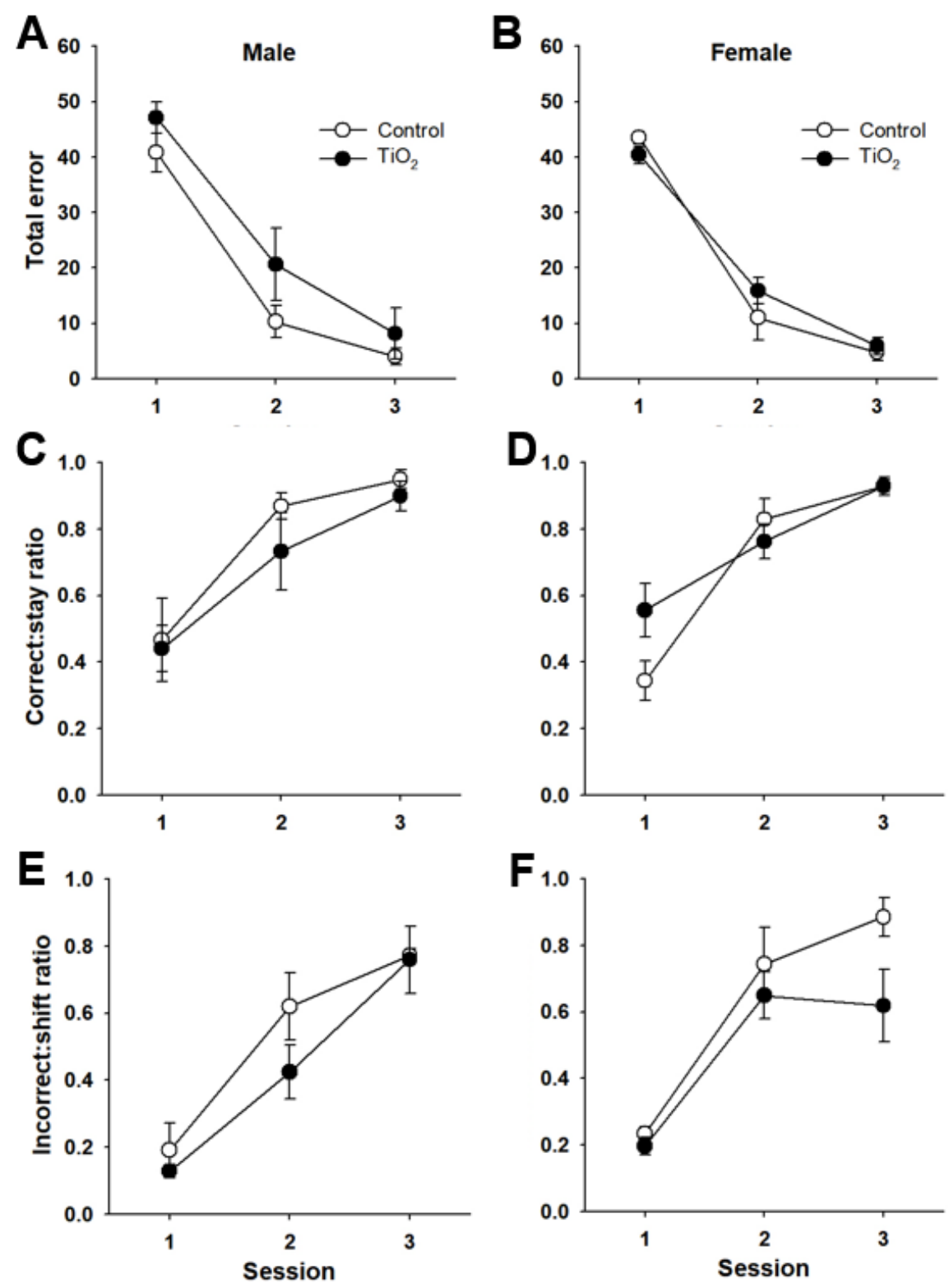

Figure 10. Mean $( \pm$ SEM) errors, correct:stay, and incorrect:shift ratios for male and female rats across the first three sessions of the first reversal. Unfilled points represent air-exposed control rats, and filled points represent nano- $\mathrm{TiO}_{2}$-exposed rats.

(Female $\mathrm{n}=6-8$; Male $\mathrm{n}=7-8$ ). 


\section{Reversal 1}
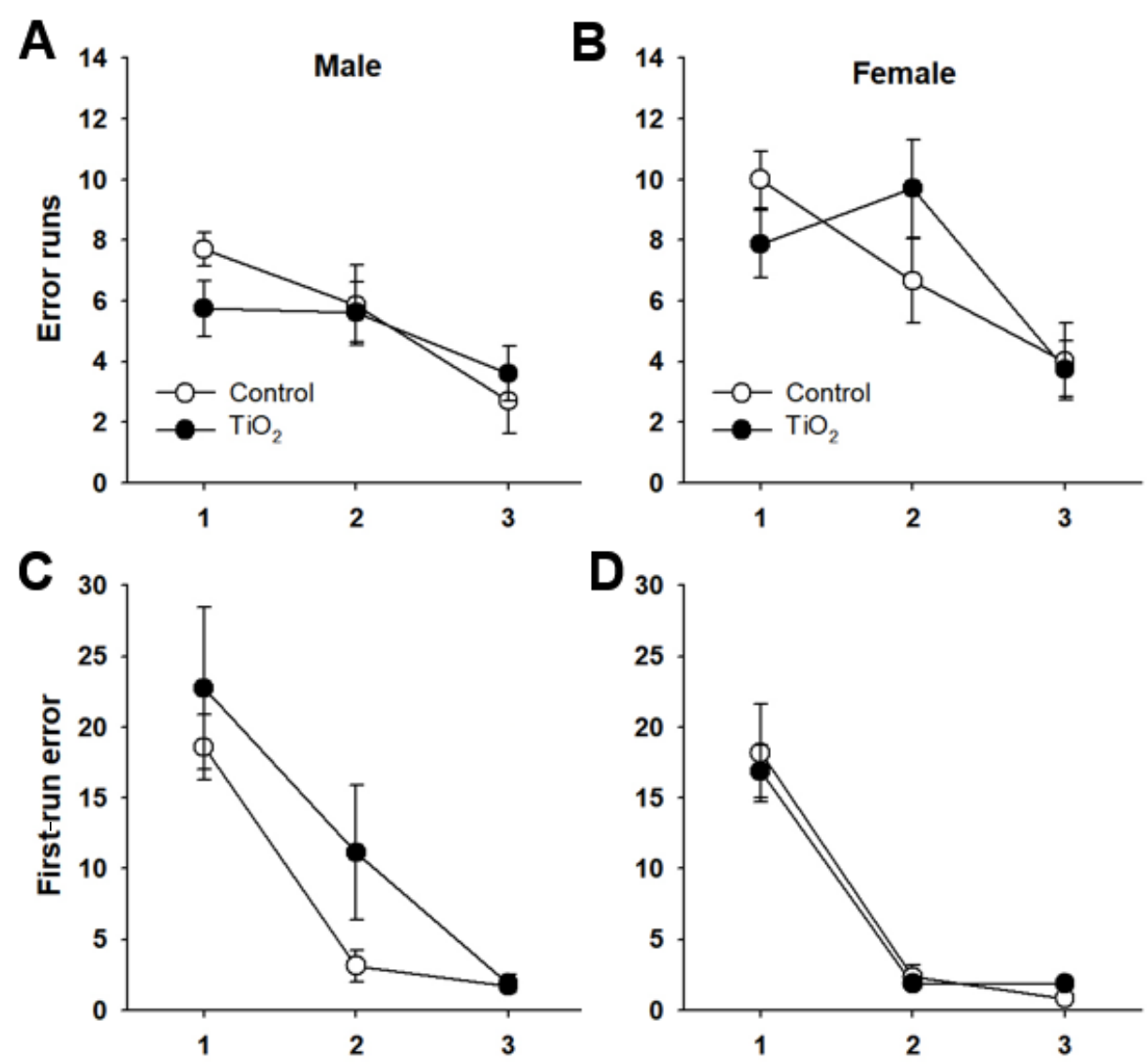

$\mathbf{D}_{30}$
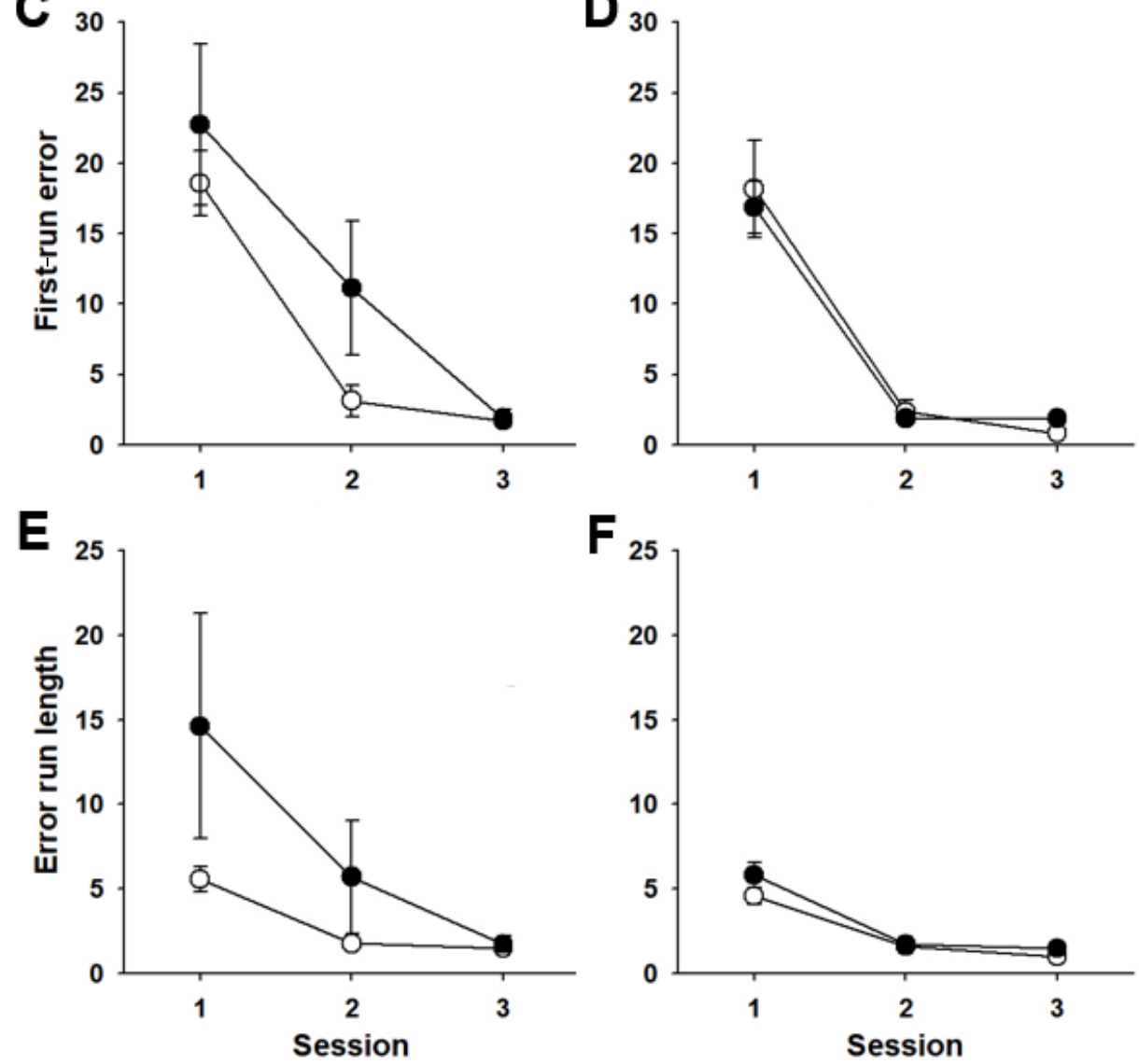

Figure 11. Mean $( \pm \mathrm{SEM})$ error runs, first-run error, and error run length for male and female rats across the first three sessions of the first reversal. Unfilled points represent air-exposed control rats, and filled points represent nano- $\mathrm{TiO}_{2}$-exposed rats.

(Female $\mathrm{n}=6-8$; Male $\mathrm{n}=7-8$ ). 


\section{Reversal 2}
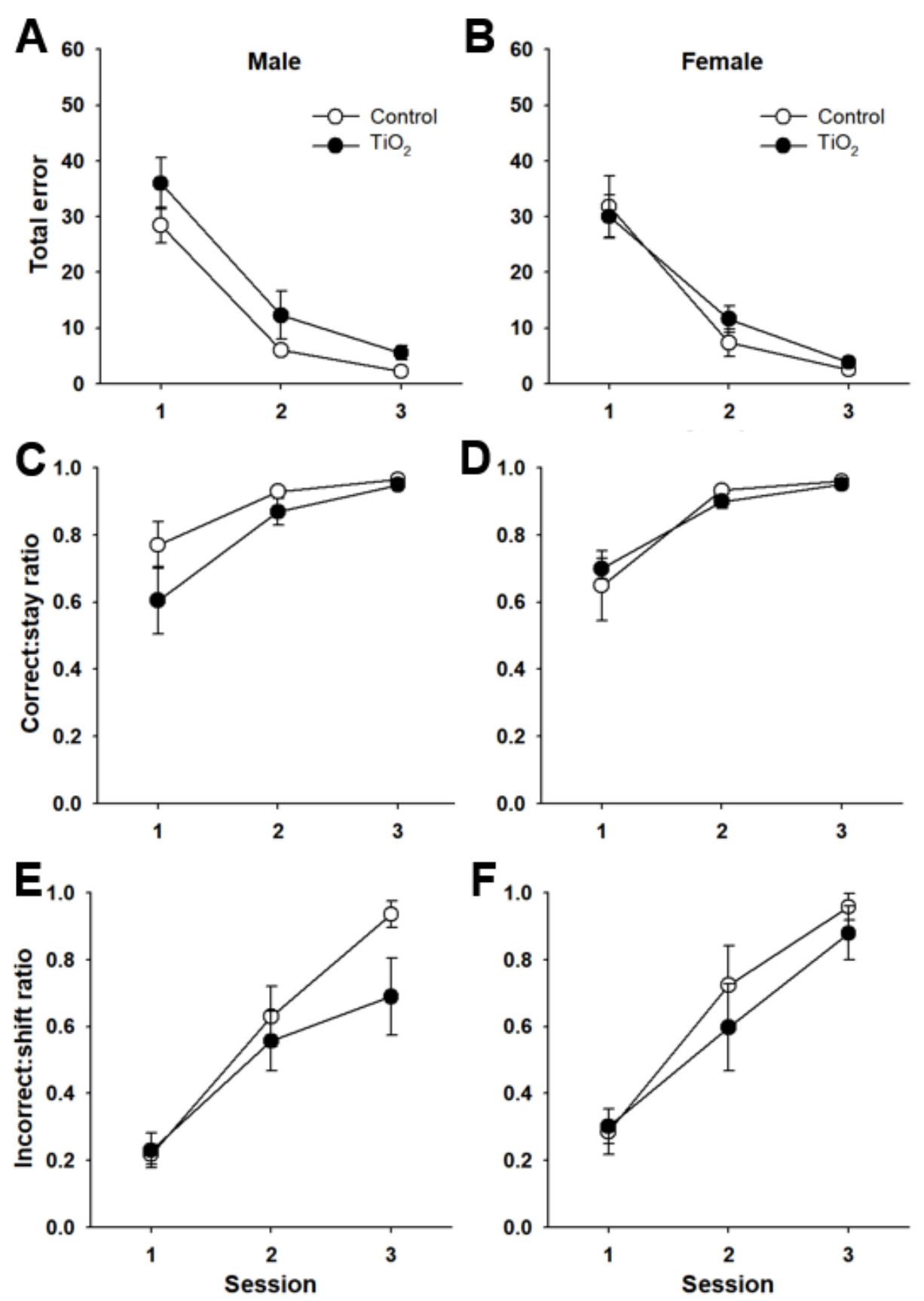

Figure 12. Mean ( \pm SEM) errors, correct:stay, and incorrect:shift ratios for male and female rats across the first three sessions of the first reversal. Unfilled points represent air-exposed control rats, and filled points represent nano- $\mathrm{TiO}_{2}$-exposed rats.

(Female $n=6-8 ;$ Male $n=7-8)$. 


\section{Reversal 2}
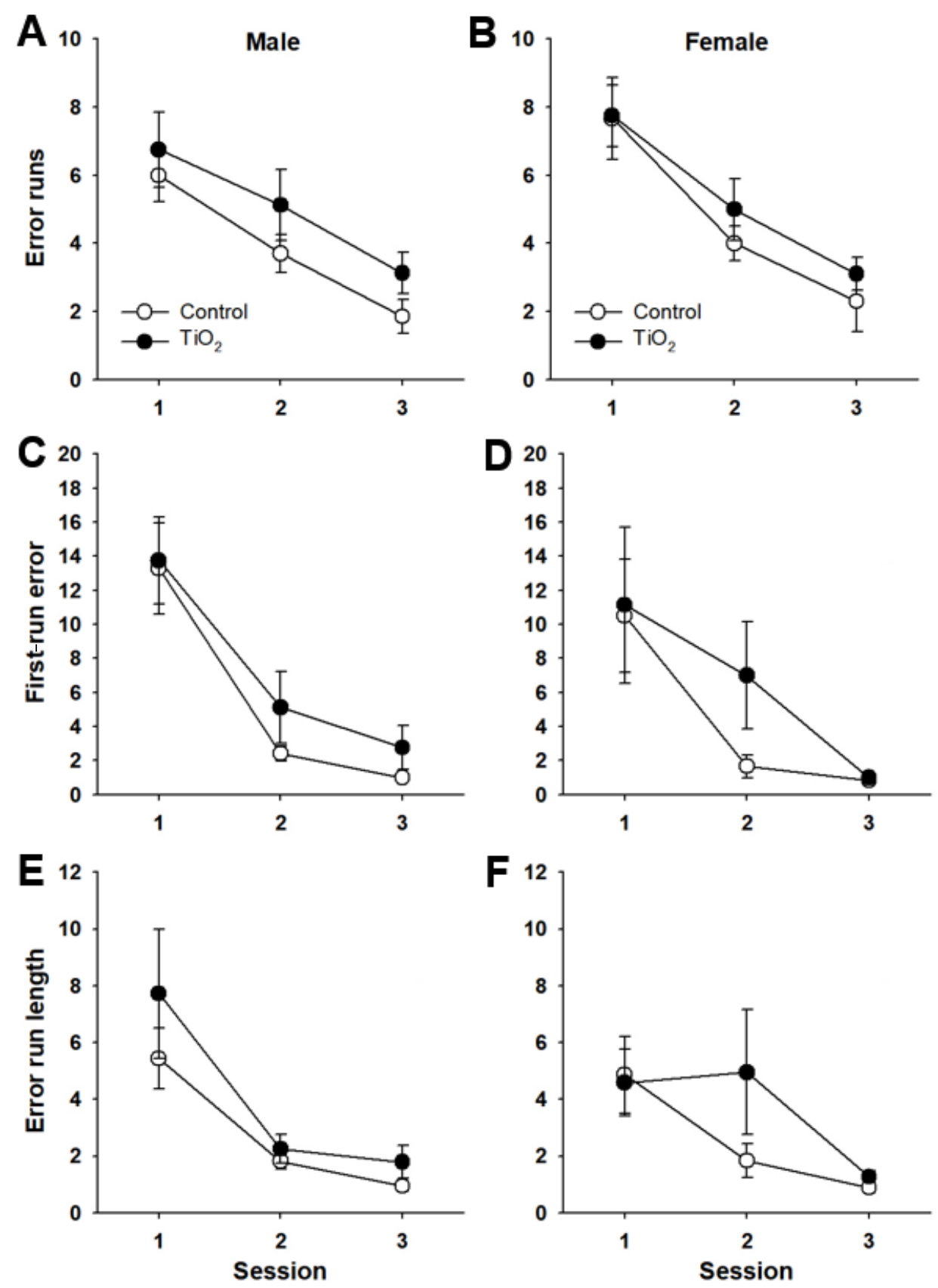

Figure 13. Mean $( \pm \mathrm{SEM})$ error runs, first-run error, and error run length for male and female rats across the first three sessions of the first reversal. Unfilled points represent air-exposed control rats, and filled points represent nano- $\mathrm{TiO}_{2}$-exposed rats.

(Female $\mathrm{n}=6-8 ;$ Male $\mathrm{n}=7-8$ ). 
DRL 5

A

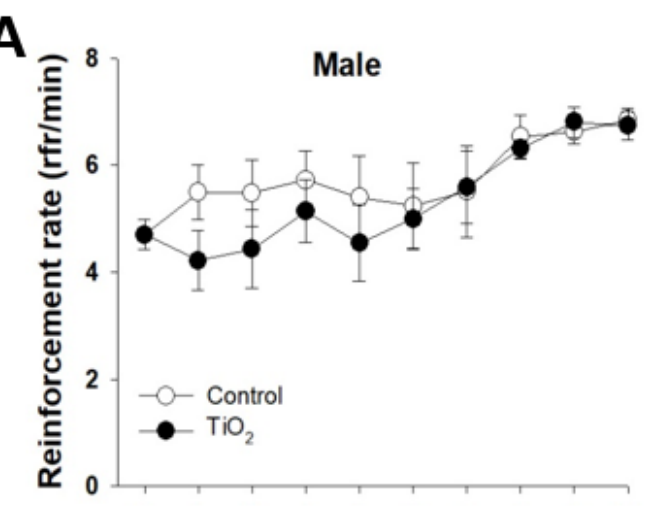

B 8

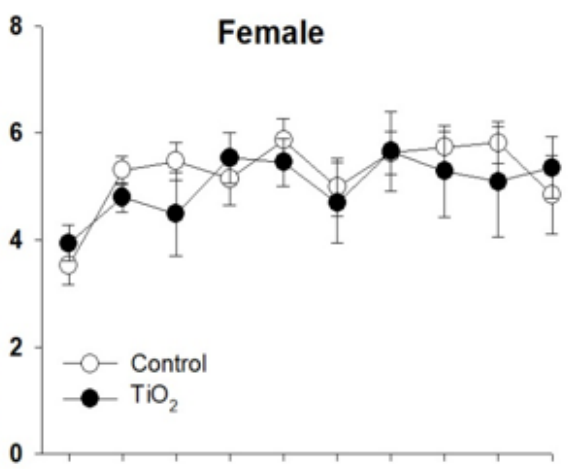

C

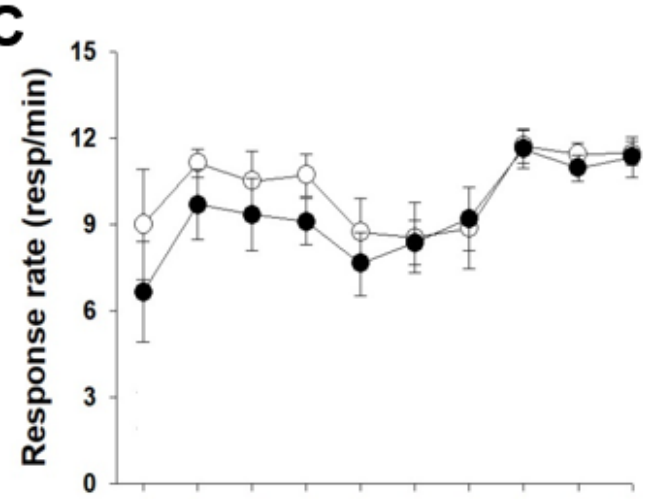

$\mathbf{D}_{15}$

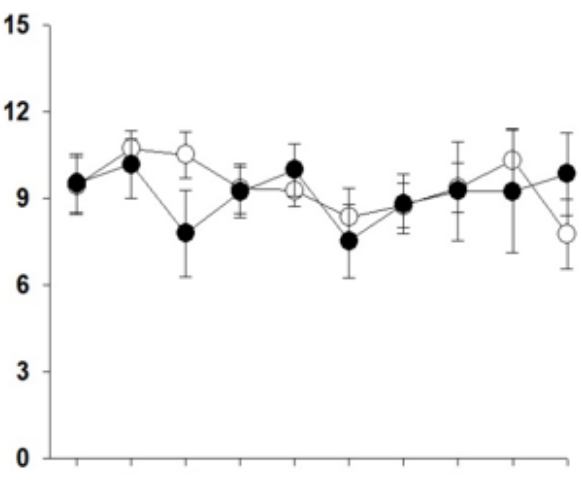

E

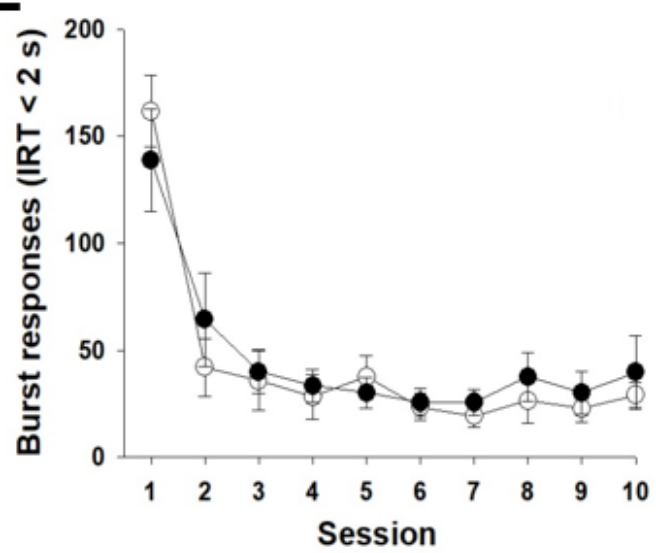

$\mathbf{F}$

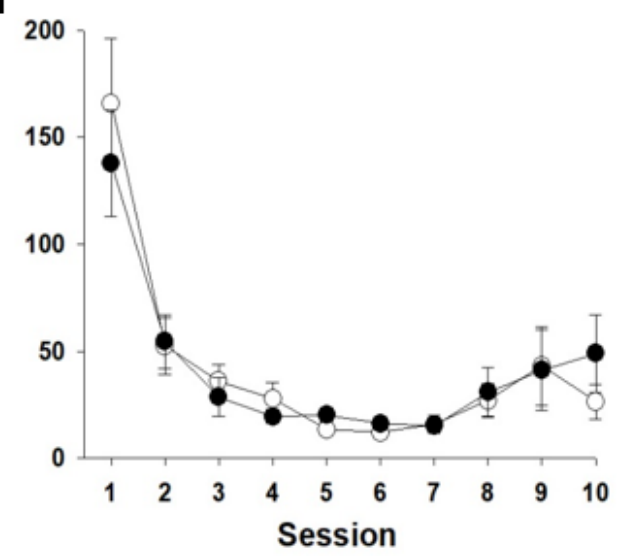

Figure 14. Mean $( \pm$ SEM) reinforcement rate, response rate, and burst responses for male and female rats across sessions on the DRL 5-s schedule. Unfilled points represent airexposed control rats, and filled points represent nano- $\mathrm{TiO}_{2}$-exposed rats.

(Female $\mathrm{n}=6-8$; Male $\mathrm{n}=7-8$ ). 


\section{DRL 5}
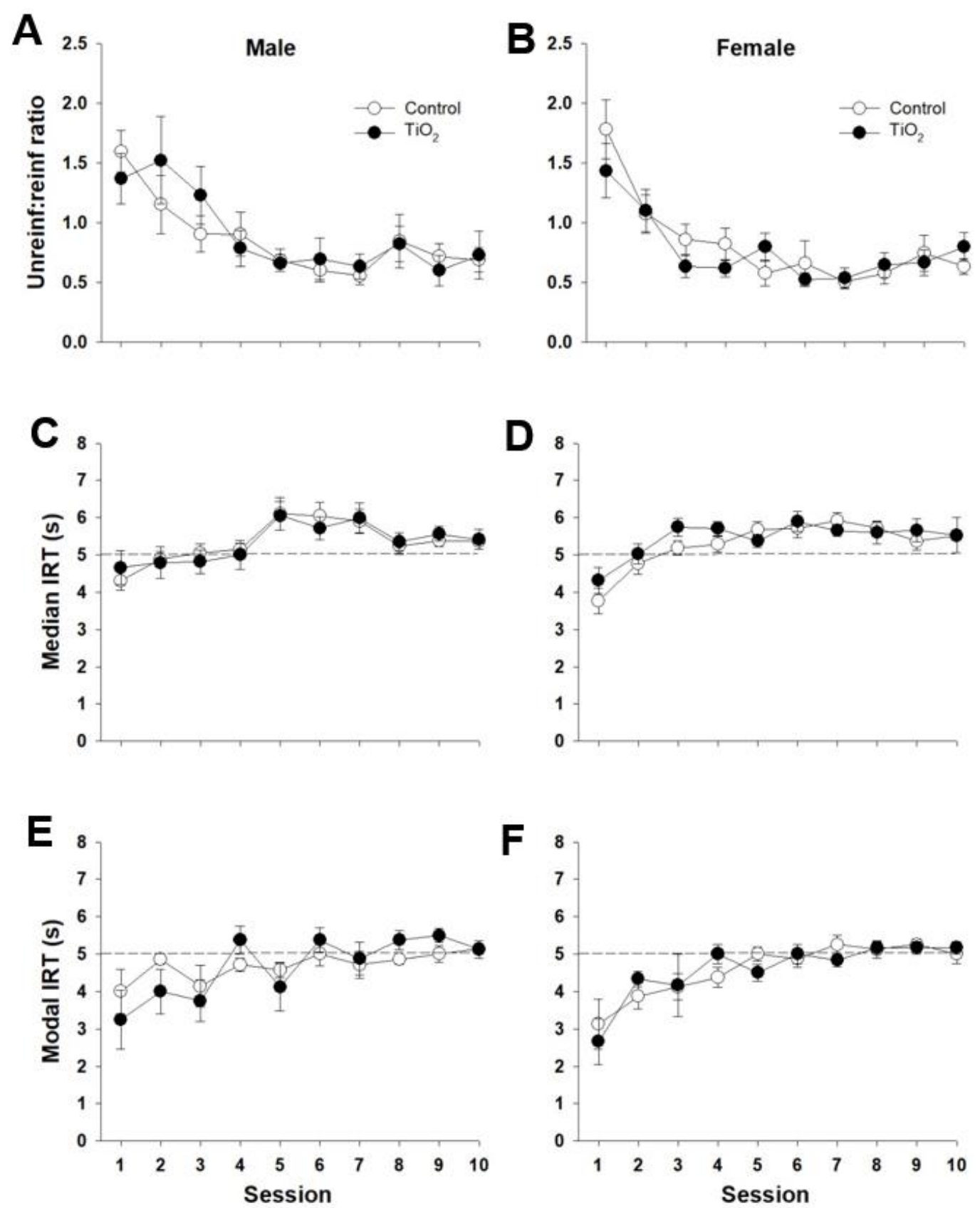

Figure 15. Mean ( \pm SEM) efficiency ratios, median and modal IRT (s) for male and female rats across sessions on the DRL 5-s schedule. The dotted lines on IRT measures indicate the criterion IRT. Unfilled points represent air-exposed control rats, and filled points represent nano- $\mathrm{TiO}_{2}$-exposed rats. (Female $\mathrm{n}=6-8$; Male $\mathrm{n}=7-8$ ). 


\section{DRL 10}

A

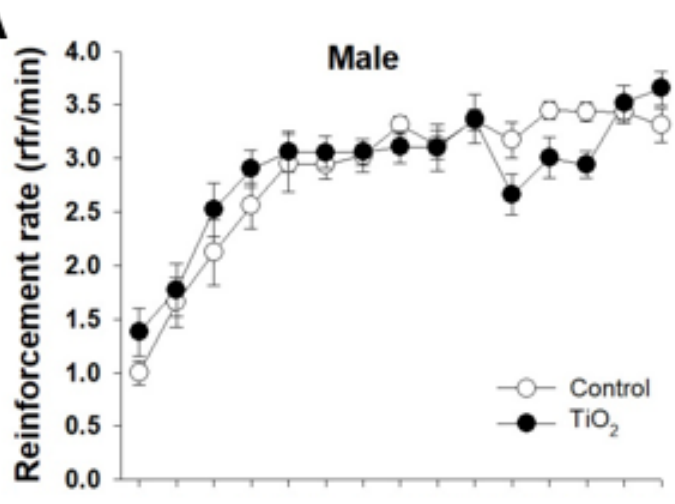

C

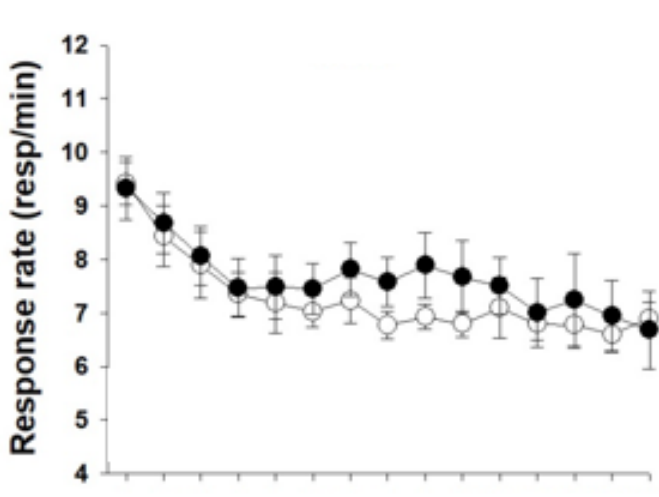

E

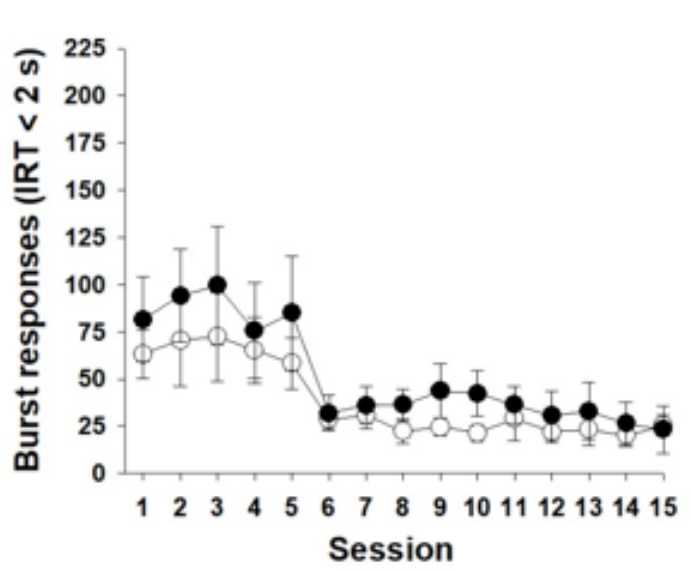

B

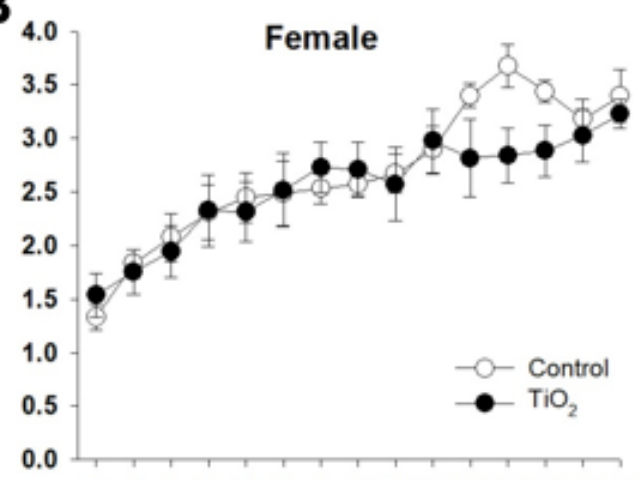

D

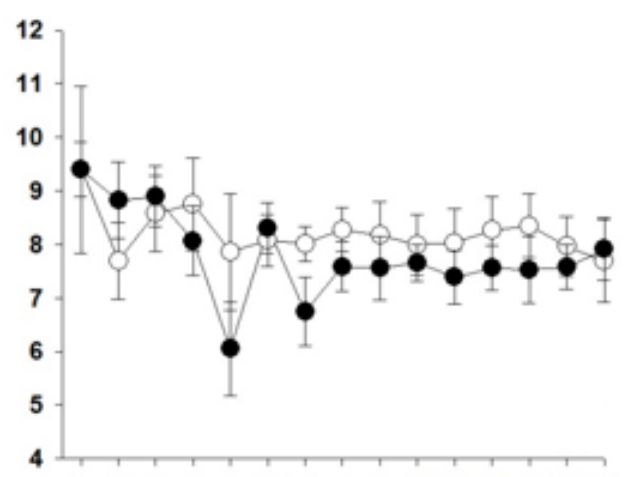

F

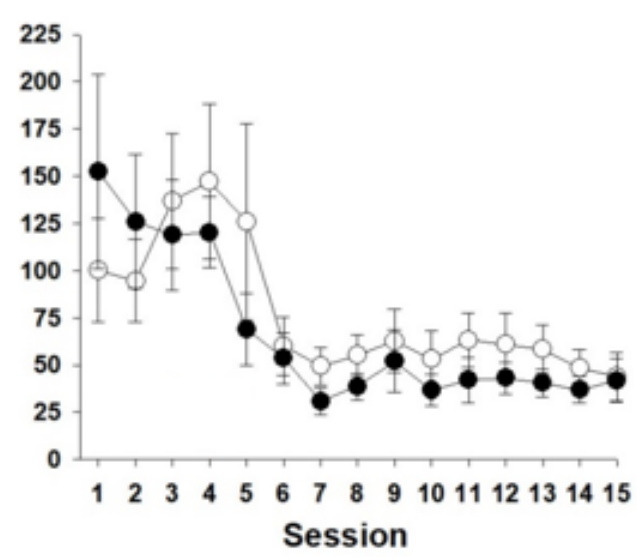

Figure 16. Mean ( $(\mathrm{SEM})$ reinforcement rate, response rate, and burst responses for male and female rats across sessions on the DRL 10-s schedule. Unfilled points represent airexposed control rats, and filled points represent nano- $\mathrm{TiO}_{2}$-exposed rats.

(Female $n=6-8$; Male $n=7-8)$. 


\section{DRL 10}

A

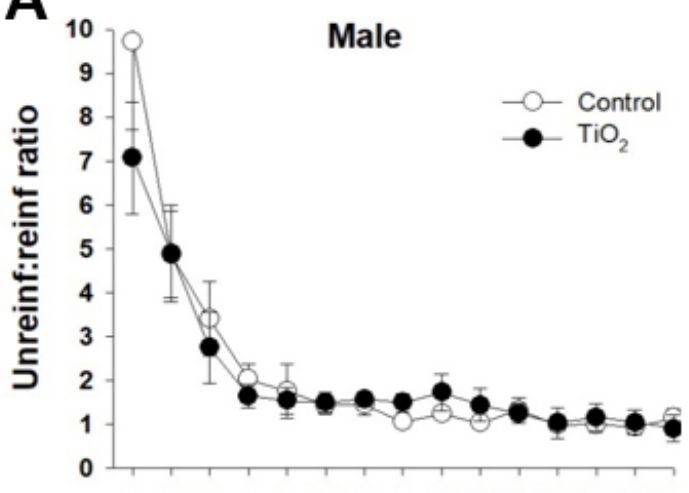

C

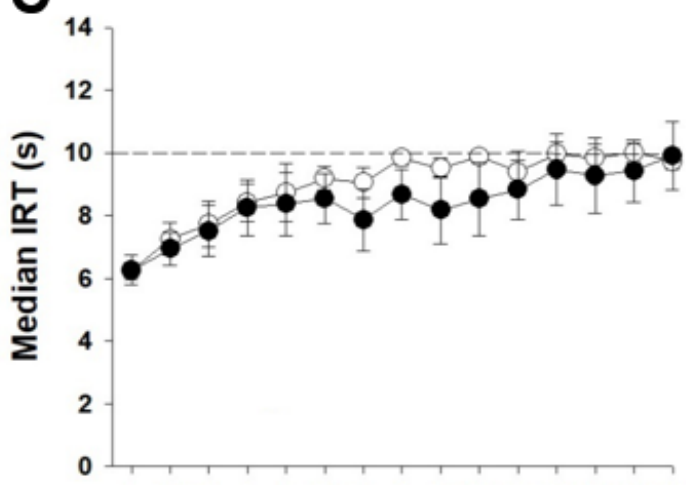

E

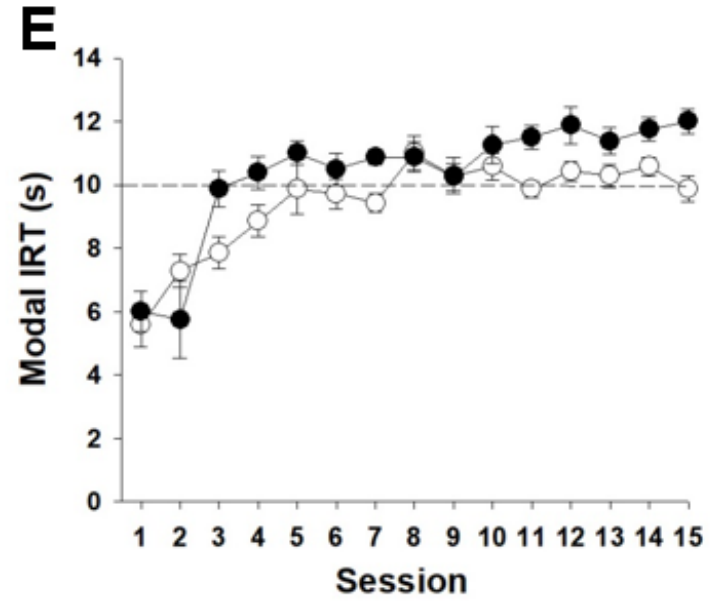

B

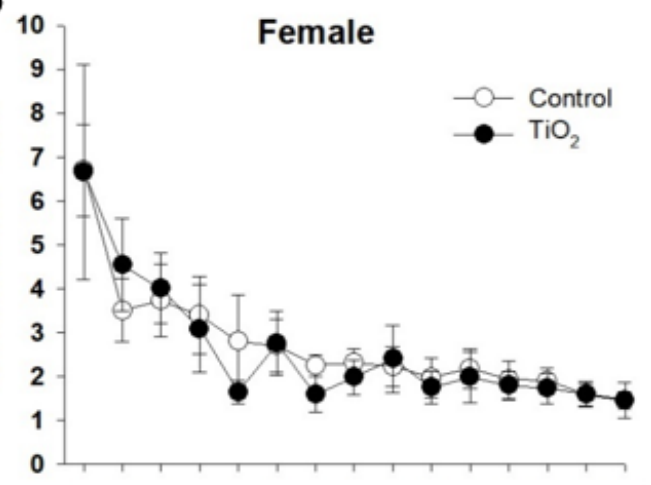

D

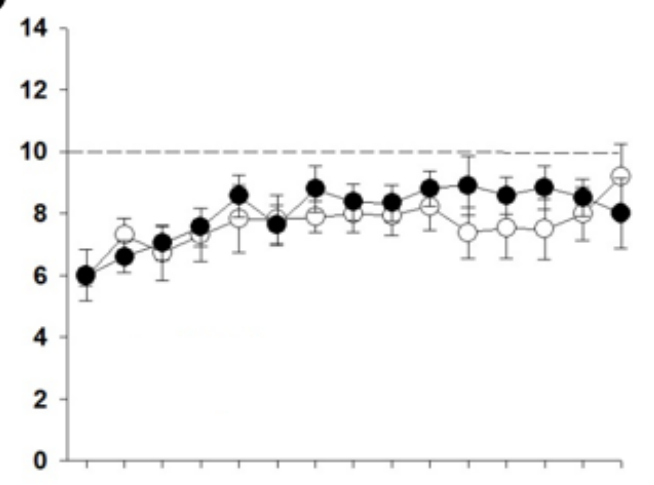

F

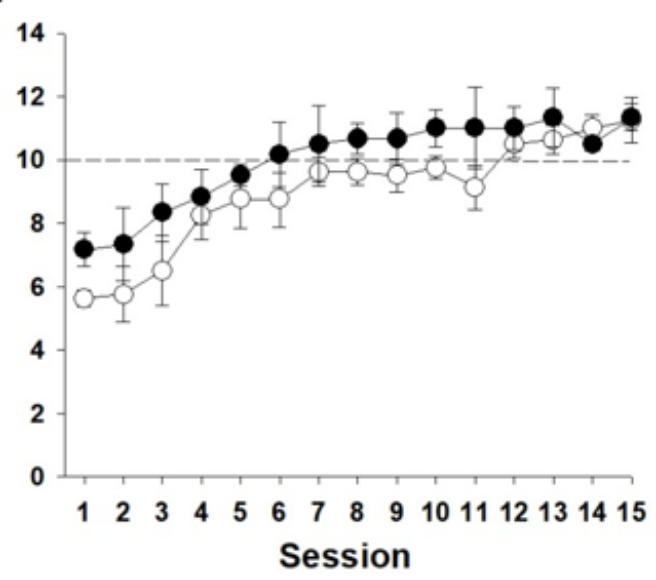

Figure 17. Mean $( \pm$ SEM) efficiency ratios, median and modal IRT (s) for male and female rats across sessions on the DRL 10-s schedule. The dotted lines on IRT measures indicate the criterion IRT. Unfilled points represent air-exposed control rats, and filled points represent nano- $\mathrm{TiO}_{2}$-exposed rats. (Female $\mathrm{n}=6-8$; Male $\mathrm{n}=7-8$ ). 
DRL 20

A

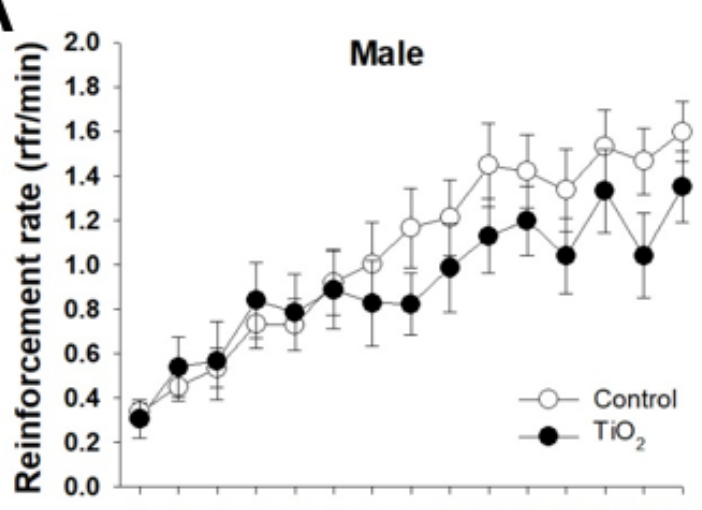

B

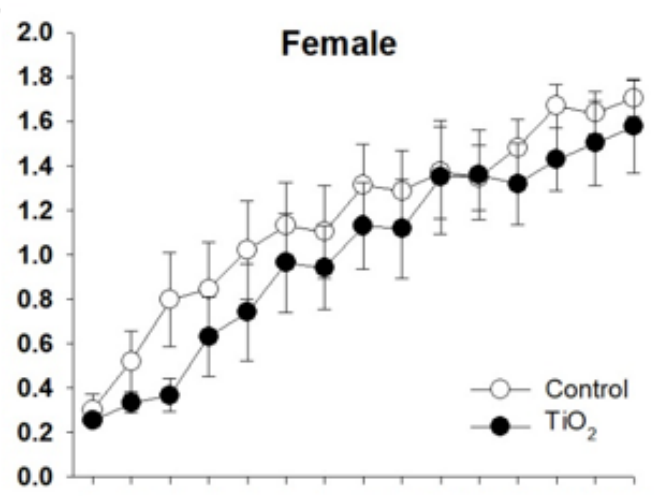

C

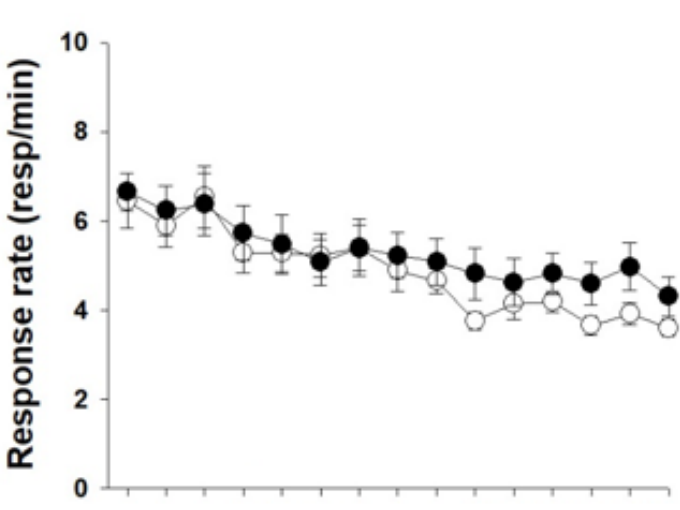

D

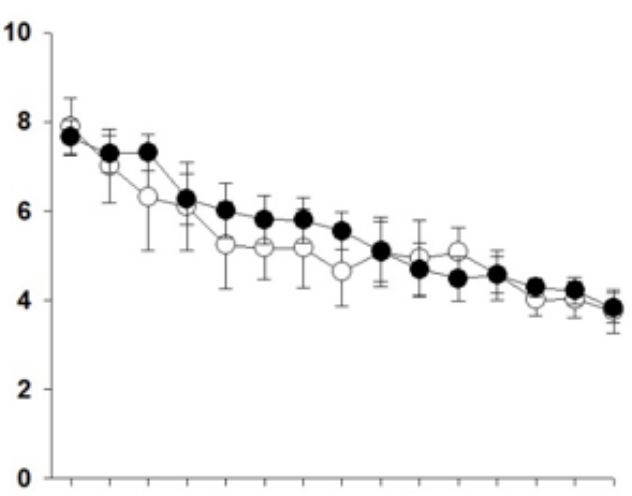

$E$
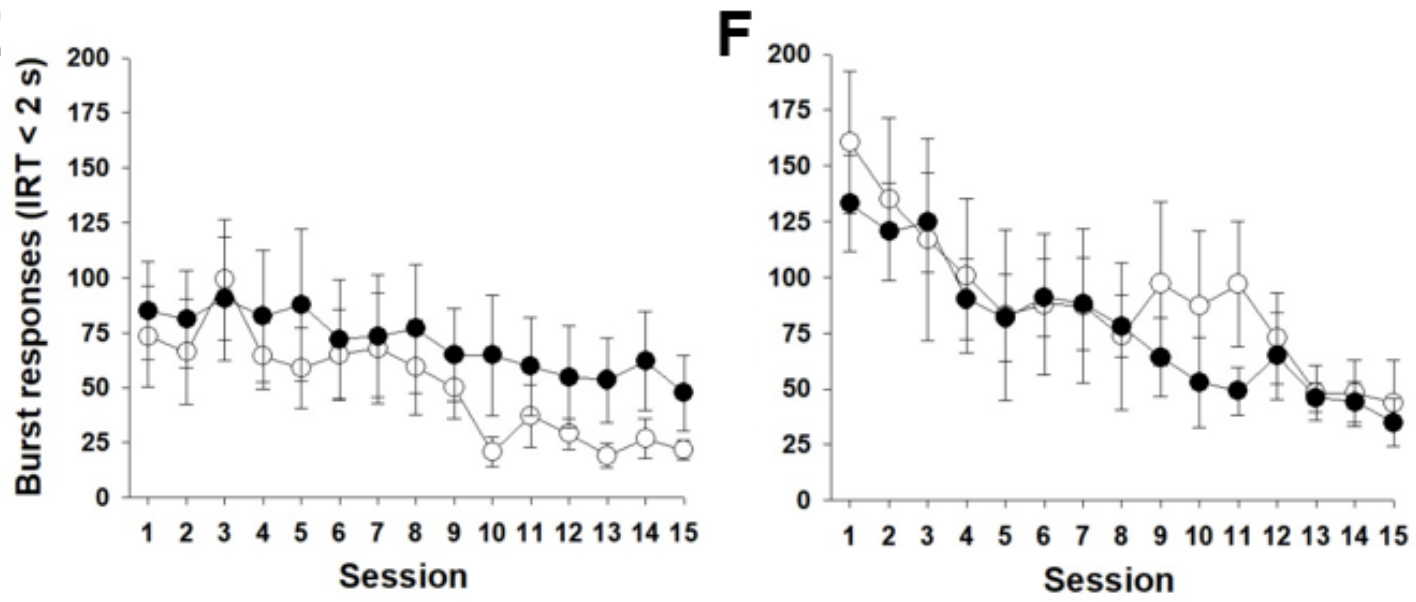

Figure 18. Mean $( \pm \mathrm{SEM})$ reinforcement rate, response rate, and burst responses for male and female rats across sessions on the DRL 10-s schedule. Unfilled points represent airexposed control rats, and filled points represent nano- $\mathrm{TiO}_{2}$-exposed rats.

(Female $\mathrm{n}=6-8$; Male $\mathrm{n}=7-8$ ). 


\section{DRL 20}
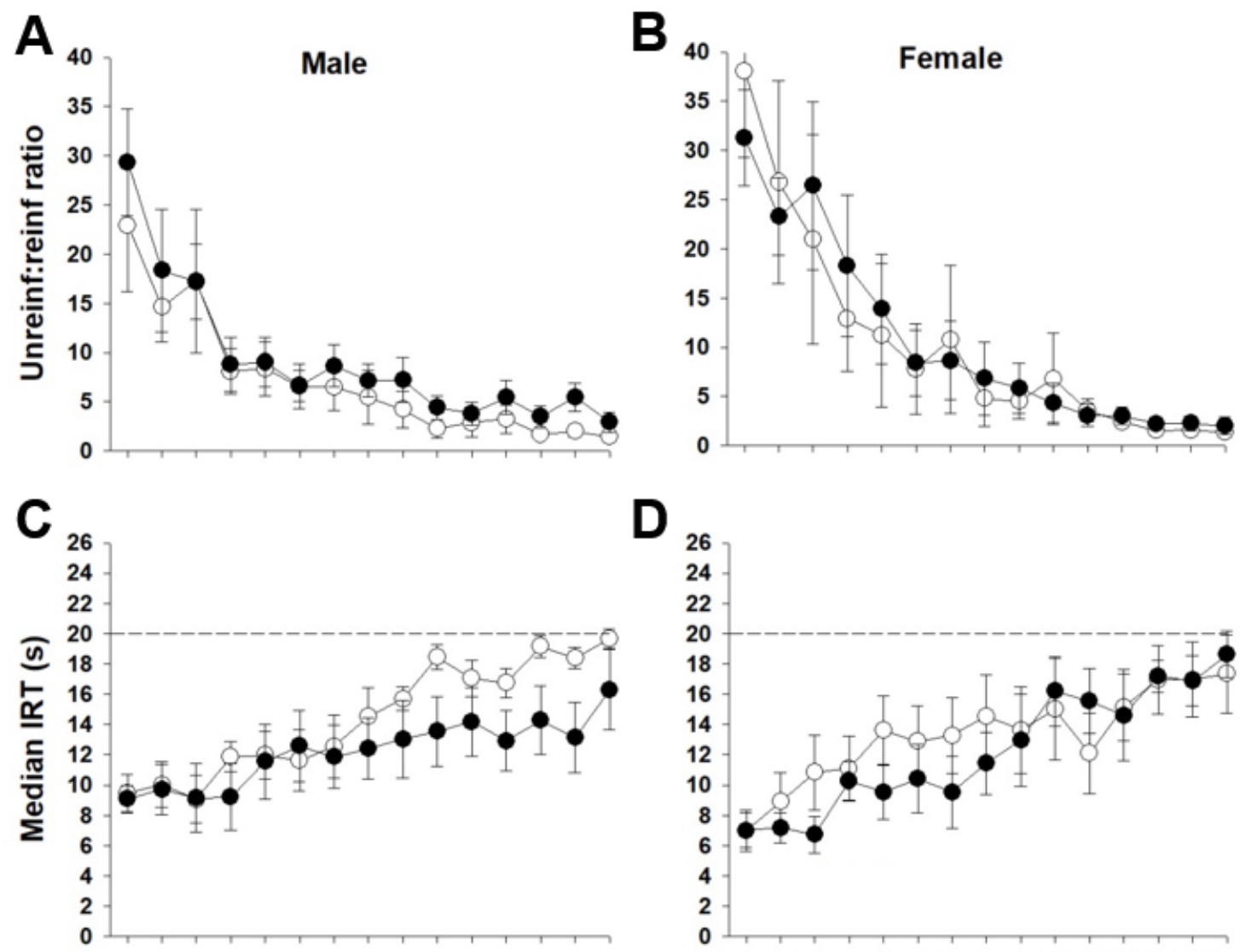

D
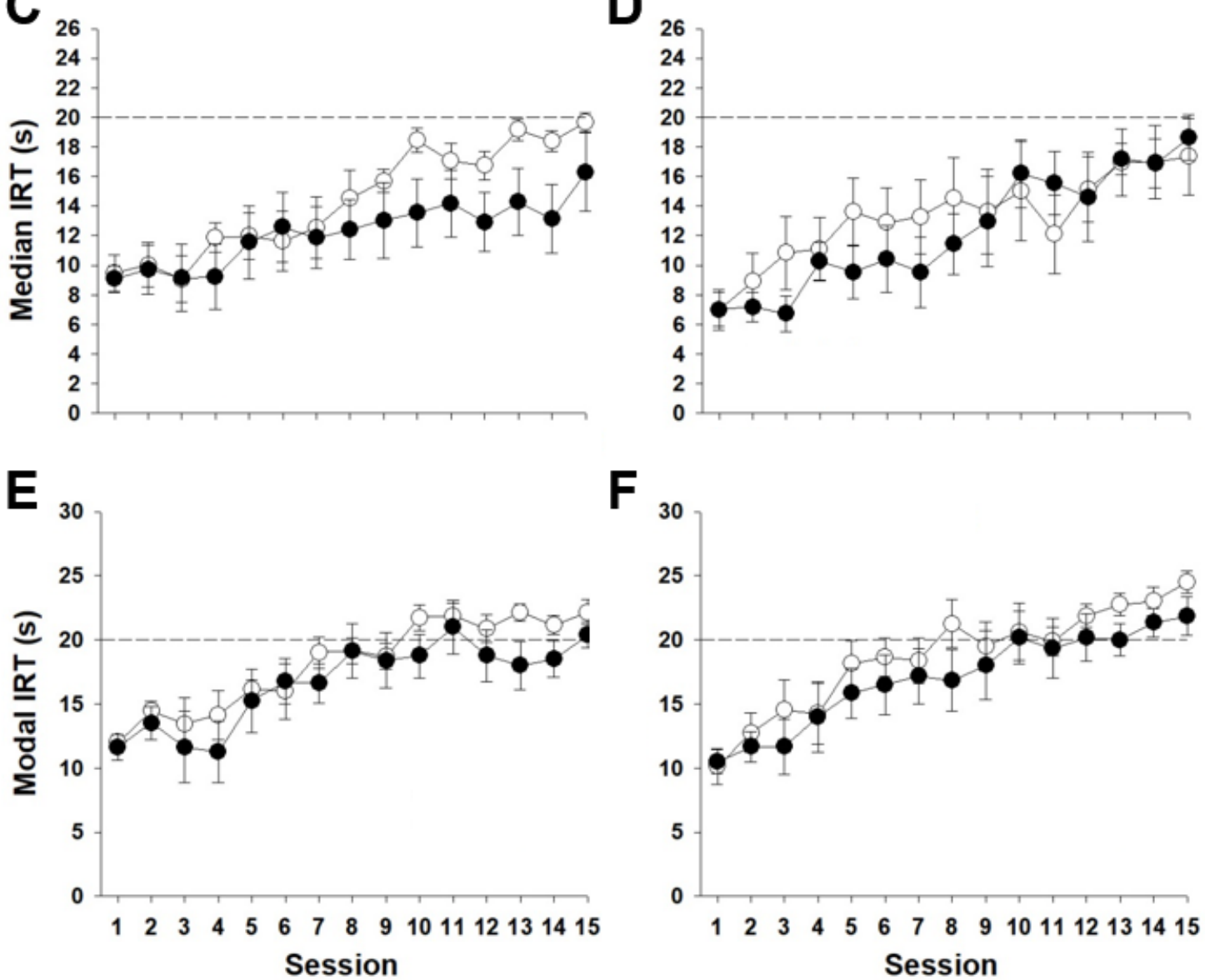

Figure 19. Mean $( \pm \mathrm{SEM})$ efficiency ratios, median and modal IRT (s) for male and female rats across sessions on the DRL 20-s schedule. The dotted lines on IRT measures indicate the criterion IRT. Unfilled points represent air-exposed control rats, and filled points represent nano- $\mathrm{TiO}_{2}$-exposed rats. (Female $\mathrm{n}=6-8$; Male $\mathrm{n}=7-8$ ). 


\section{DRL 30}

A

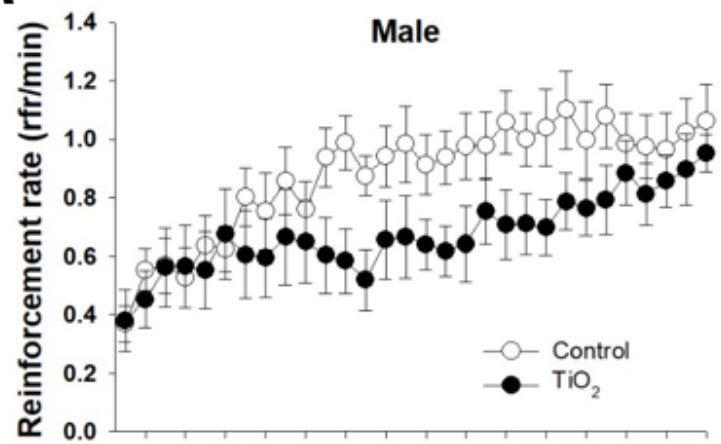

C

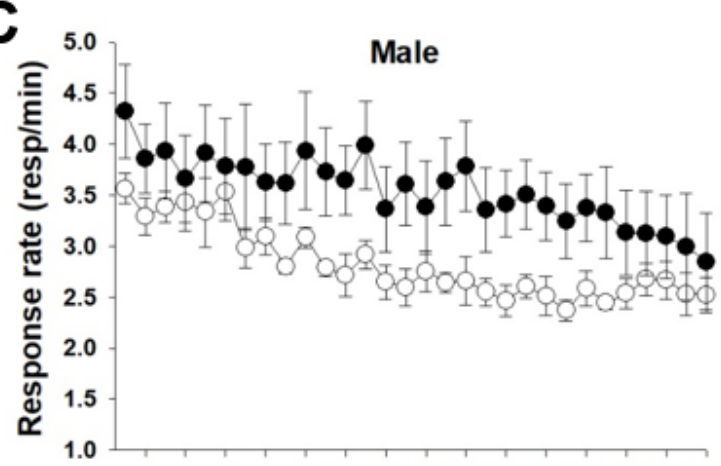

E

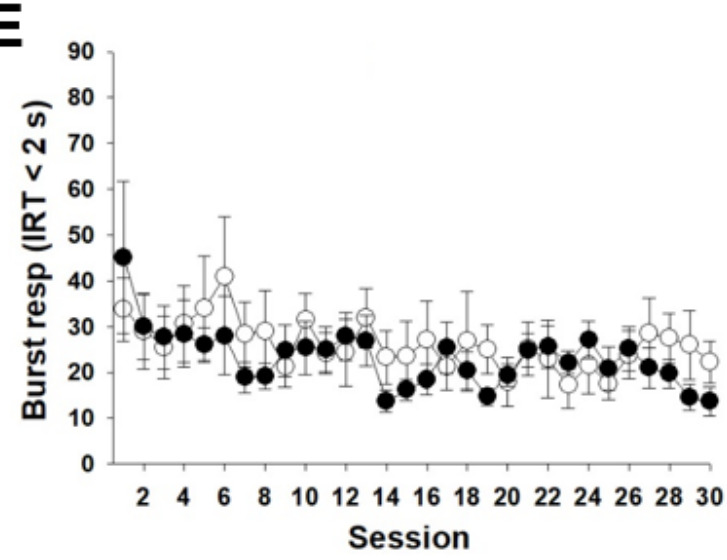

B

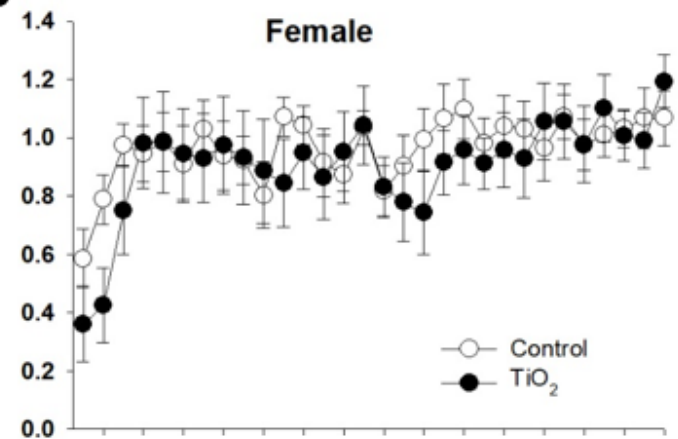

D

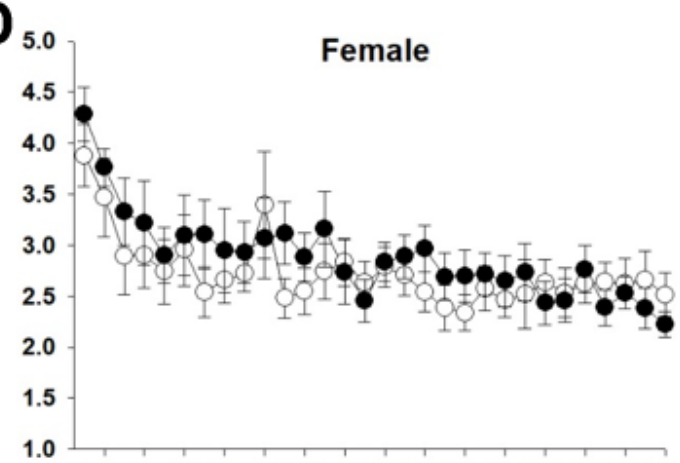

$\mathbf{F}$

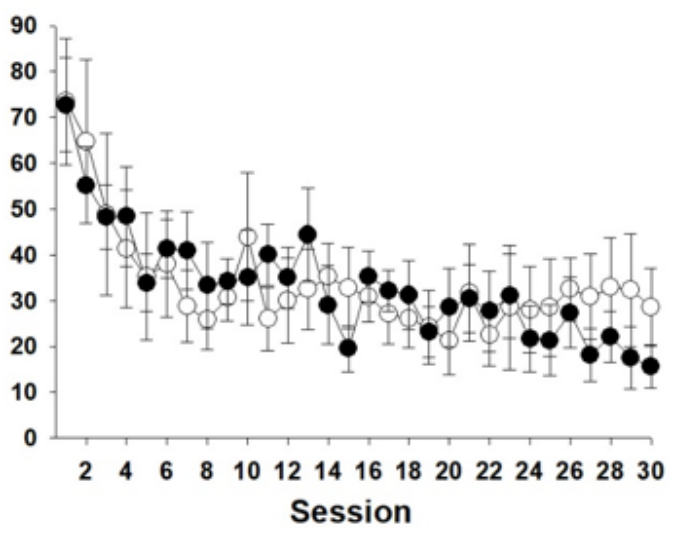

Figure 20. Mean ( \pm SEM) reinforcement rate, response rate, and burst responses for male and female rats across sessions on the DRL 30-s schedule. Unfilled points represent airexposed control rats, and filled points represent nano- $\mathrm{TiO}_{2}$-exposed rats.

(Female $\mathrm{n}=6-8$; Male $\mathrm{n}=7-8$ ). 
DRL 30

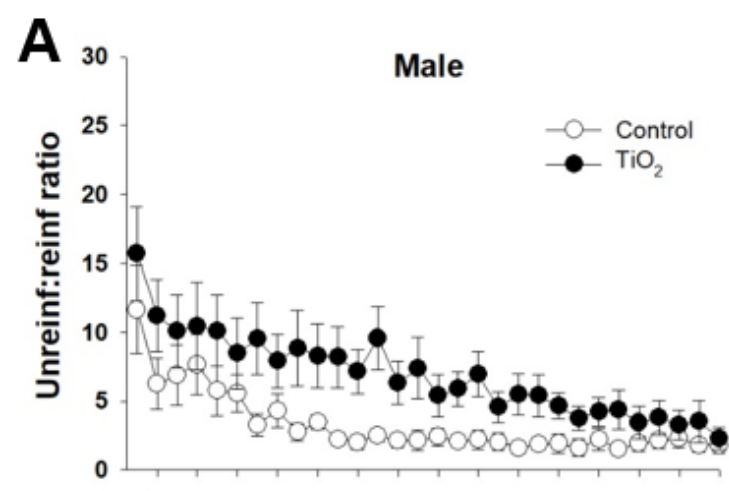

$\mathbf{B}_{30}$
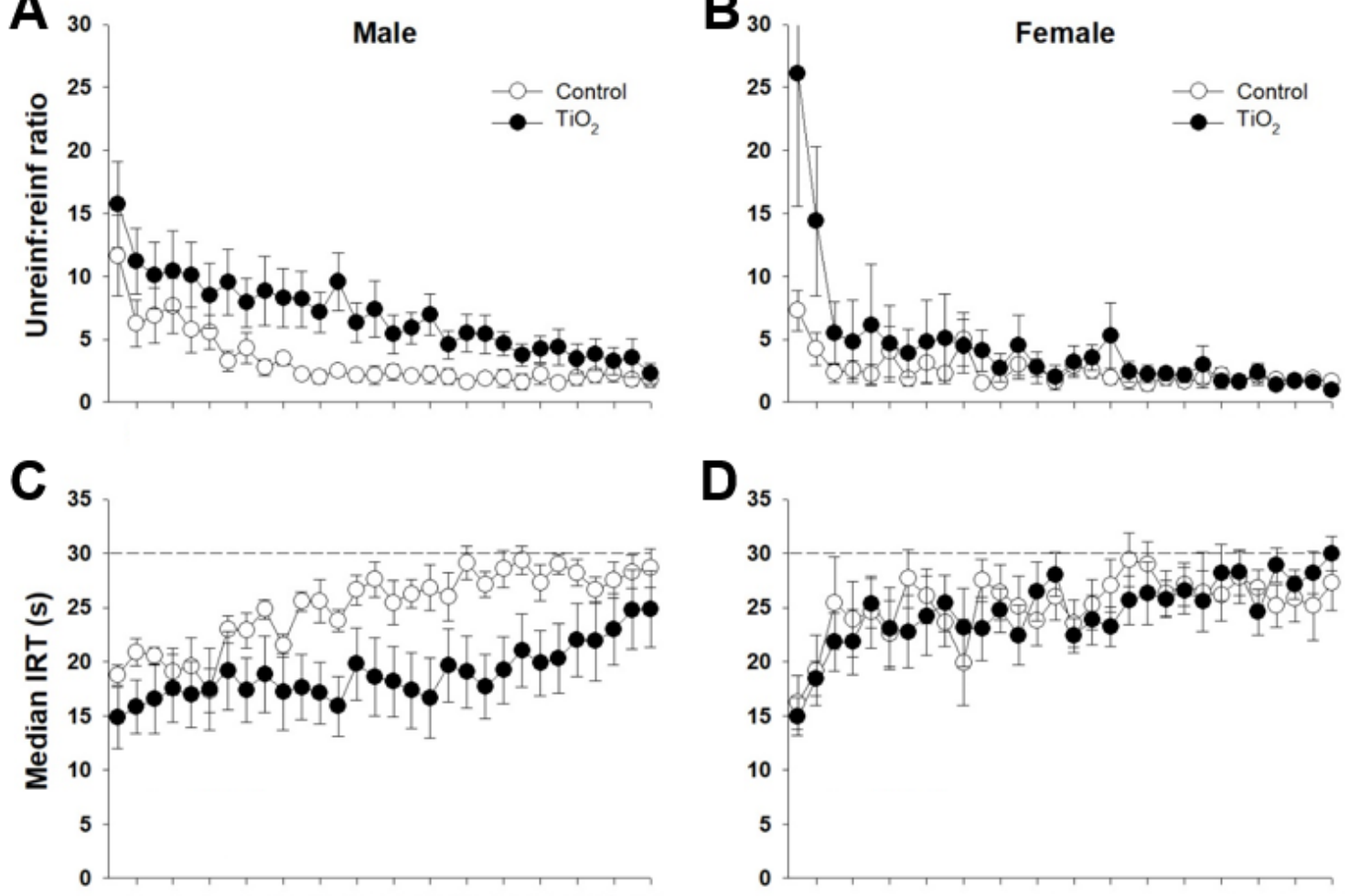

$\mathbf{D}_{3}$
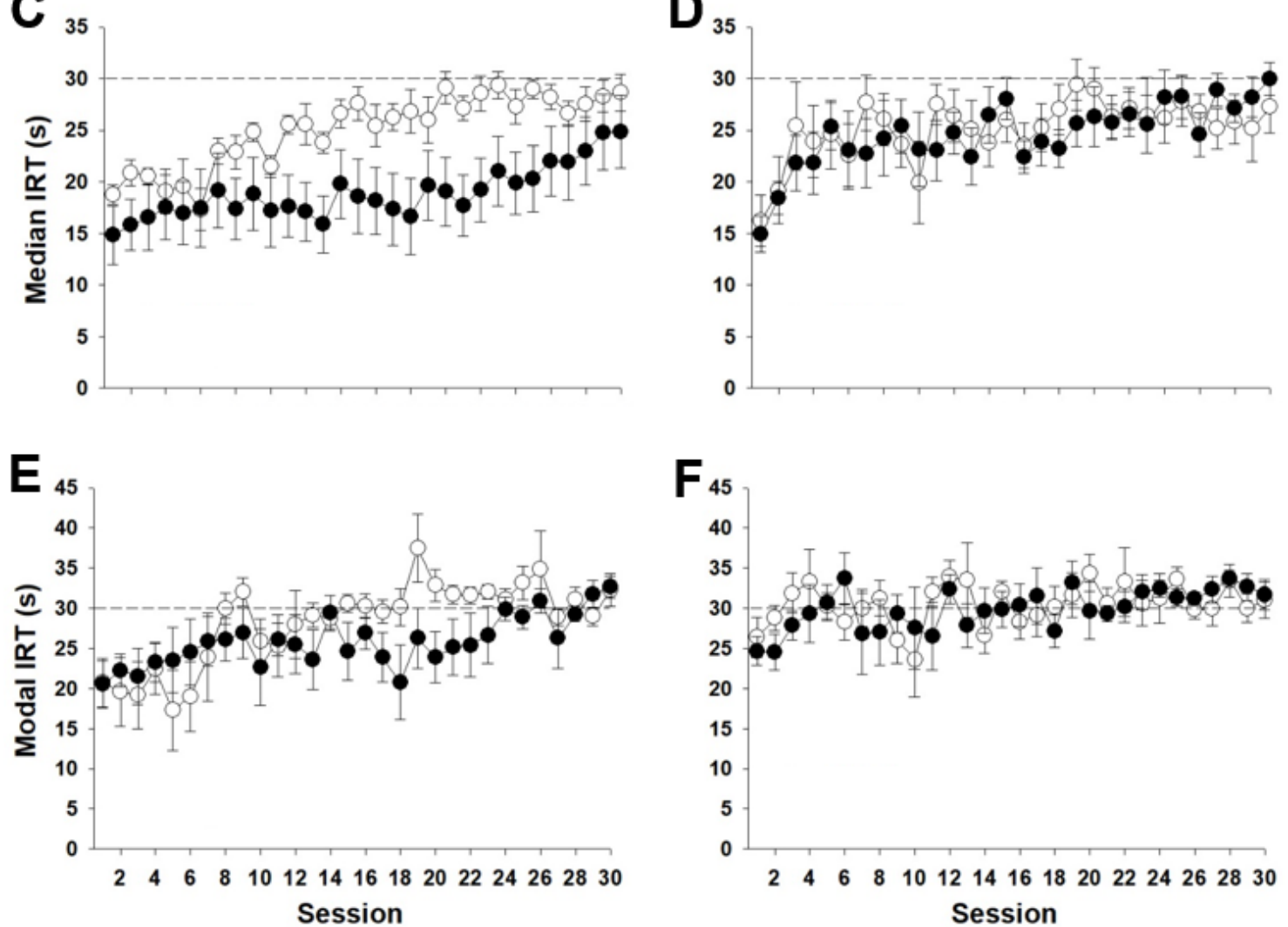

Figure 21. Mean ( \pm SEM) efficiency ratios, median and modal IRT (s) for male and female rats across sessions on the DRL 30-s schedule. The dotted lines on IRT measures indicate the criterion IRT. Unfilled points represent air-exposed control rats, and filled points represent nano- $\mathrm{TiO}_{2}$-exposed rats. (Female $\mathrm{n}=6-8$; Male $\mathrm{n}=7-8$ ). 

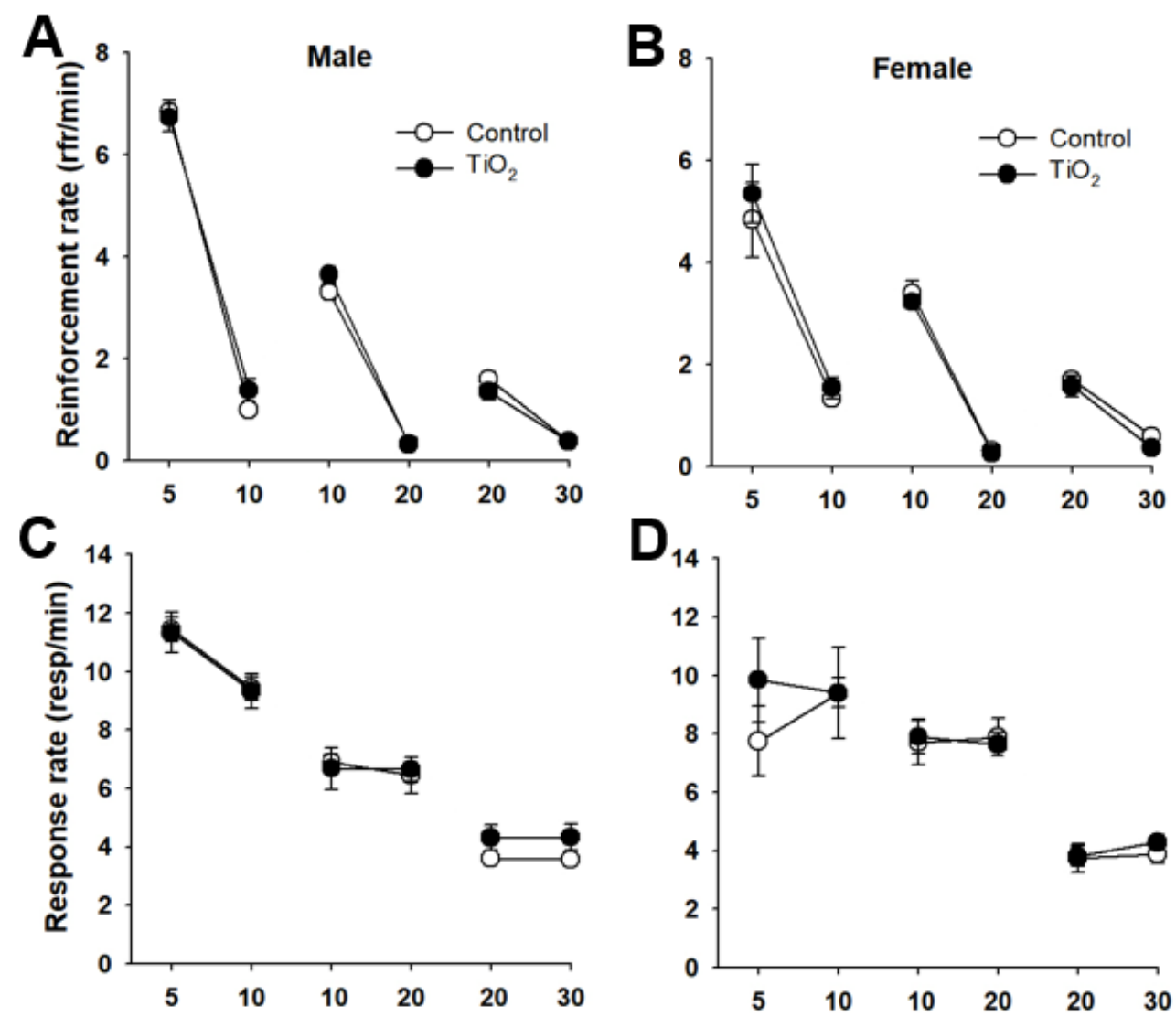

$\mathbf{D}_{14}$
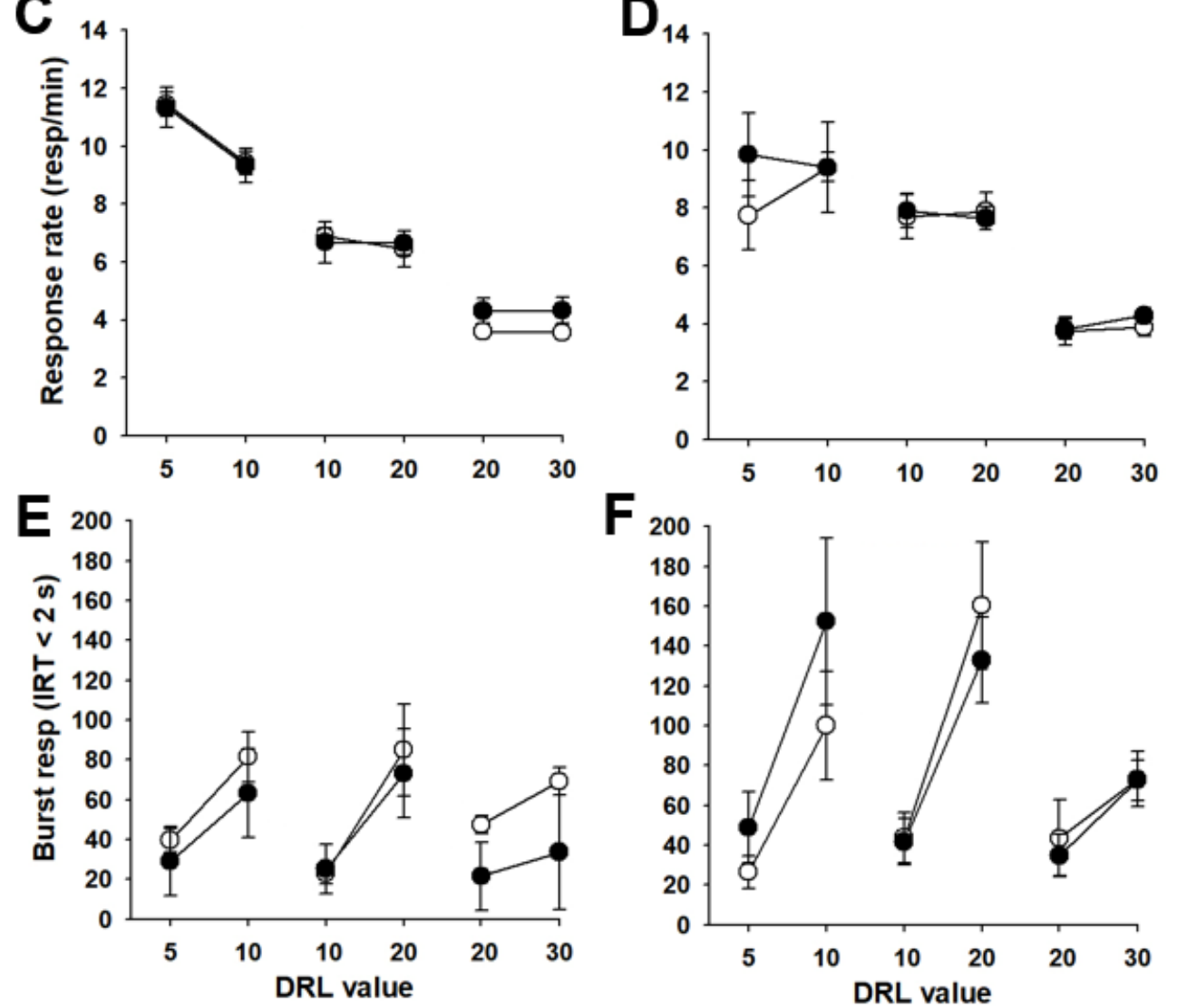

Figure 22. Mean $( \pm \mathrm{SEM})$ reinforcement rate, response rate, and burst responses for male and female rats across all DRL transitions. Transitions encompassed the last session of the previous DRL schedule and the first session of the upcoming DRL schedule. Unfilled points represent air-exposed control rats, and filled points represent nano- $\mathrm{TiO}_{2}$-exposed rats. (Female $n=6-8$; Male $n=7-8$ ). 

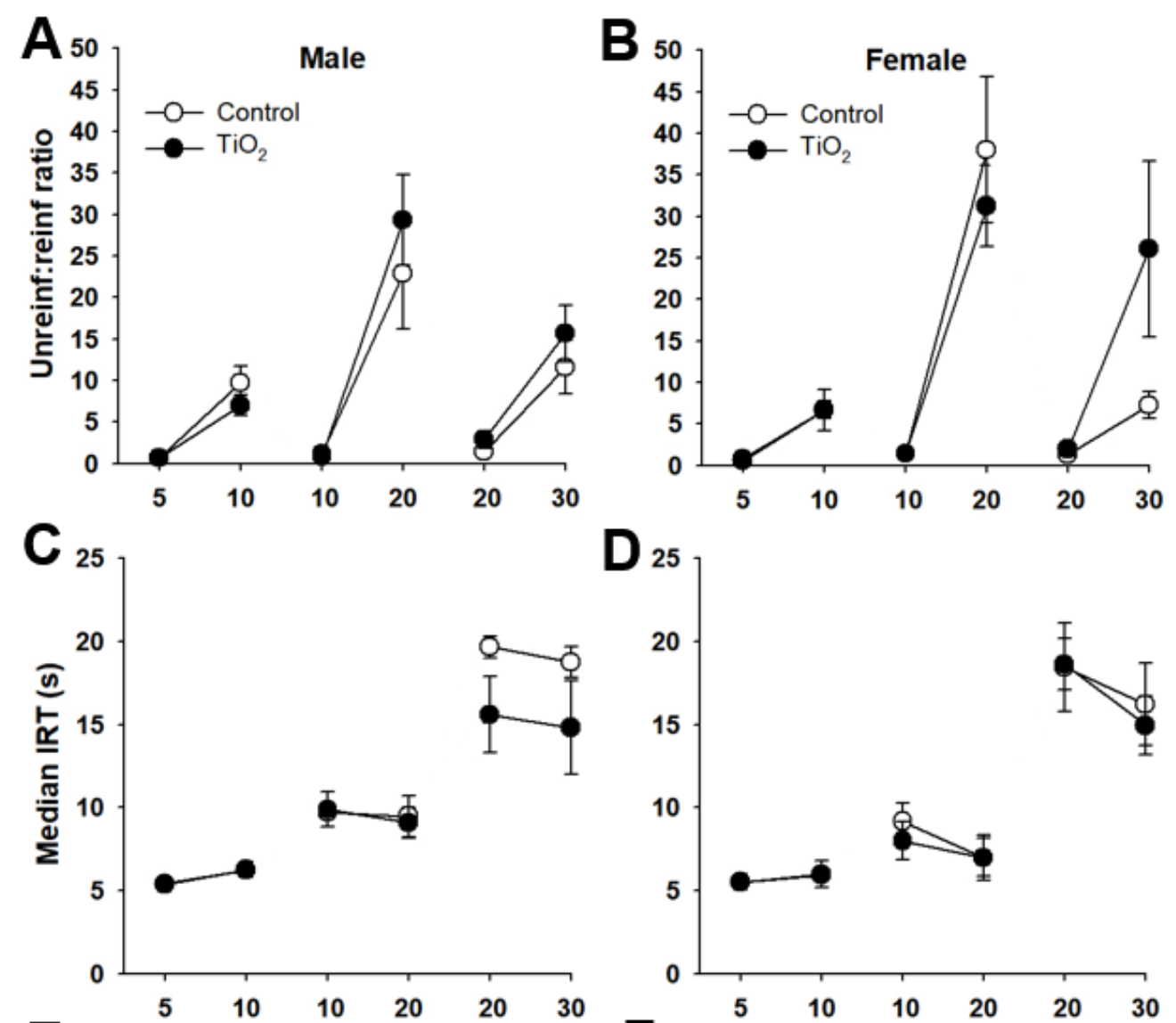

$\mathbf{D}_{25}$
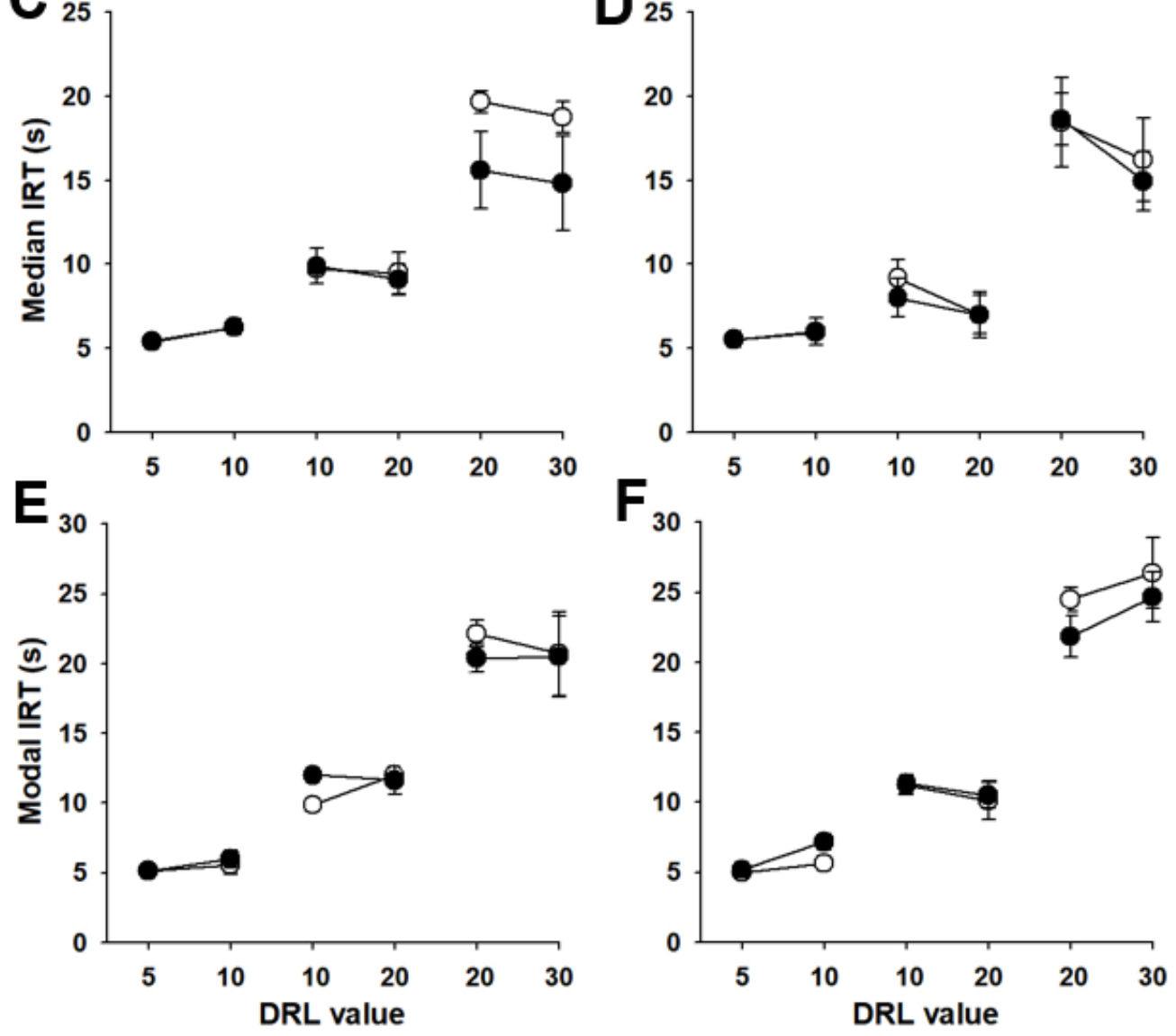

Figure 23. Mean ( \pm SEM) efficiency ratios, median IRT, and modal IRT for male and female rats across all DRL transitions. Transitions encompassed the last session of the previous DRL schedule and the first session of the upcoming DRL schedule. Unfilled points represent air-exposed control rats, and filled points represent nano- $\mathrm{TiO}_{2}$-exposed rats. (Female $\mathrm{n}=6-8$; Male $\mathrm{n}=7-8$ ). 
Appendix A

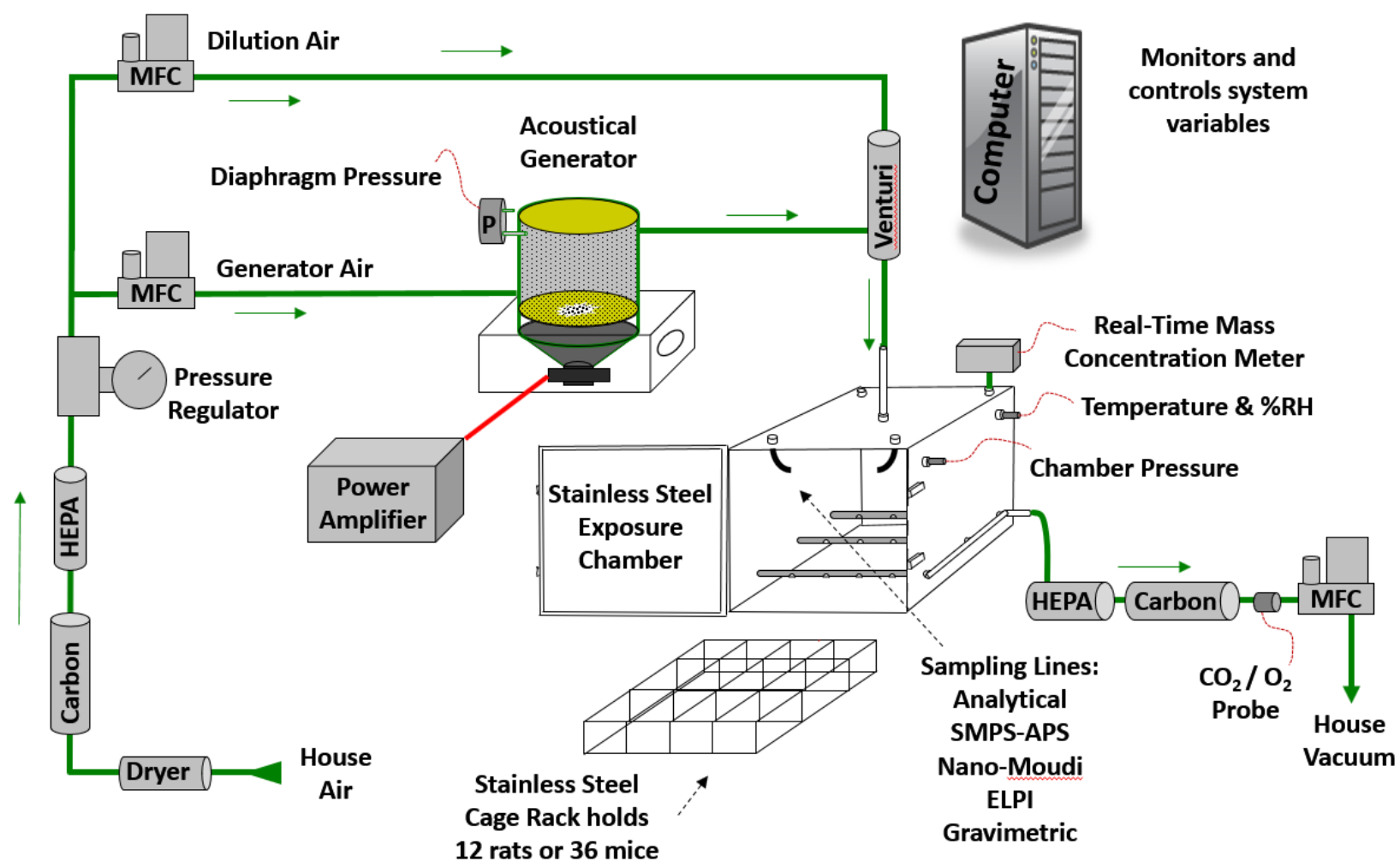

Figure A. Schematic of the acoustical generator aerosol exposure system used for nano$\mathrm{TiO}_{2}$ exposures. 


\section{Appendix B}
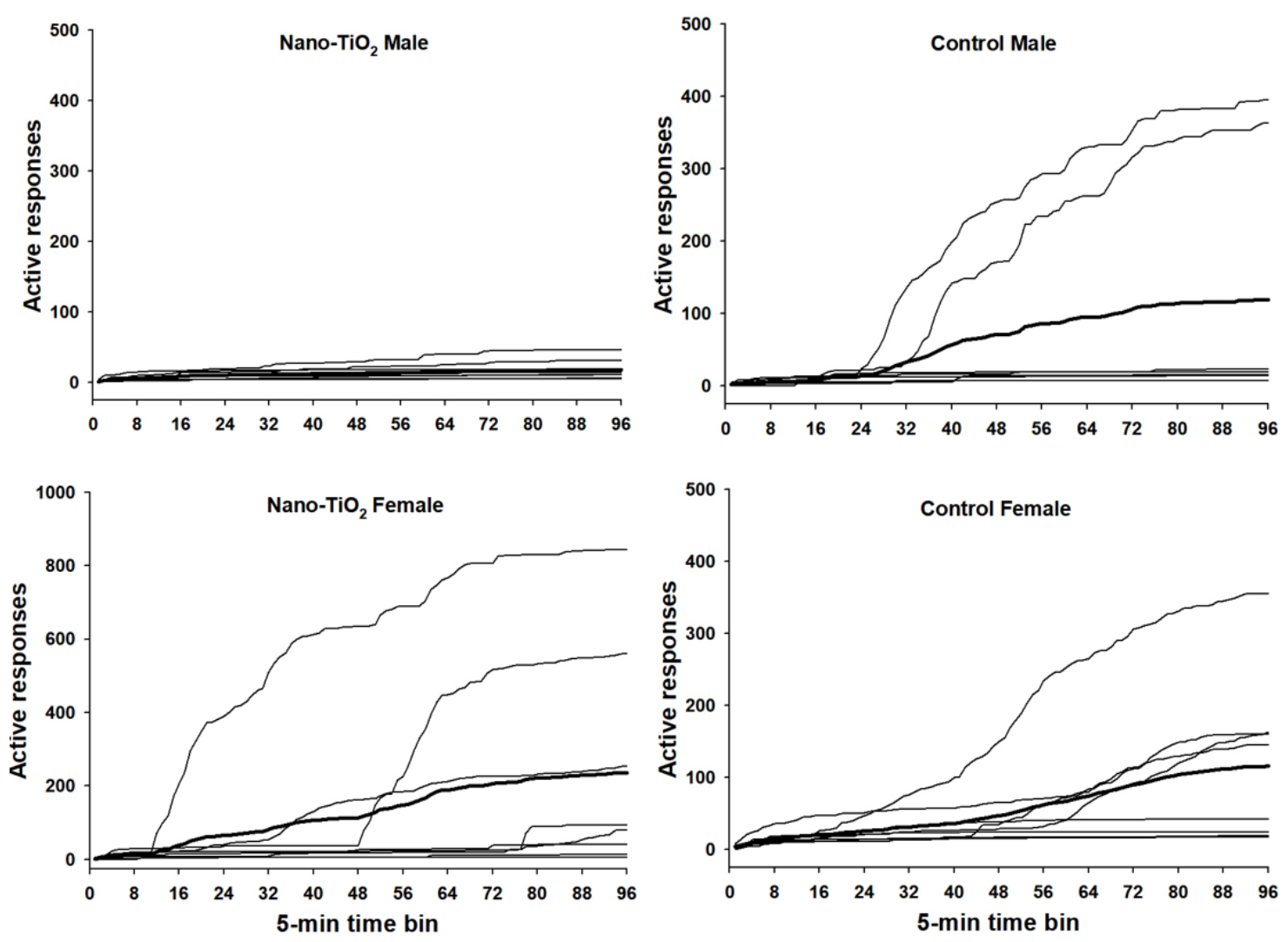

Figure B. Cumulative response records for active lever responses for all rats during the 8$\mathrm{h}$ acquisition session. Bolded lines in each panel represent the average of all rats within that group. (Female $\mathrm{n}=8$; Male $\mathrm{n}=7-8$ ) 
Appendix C

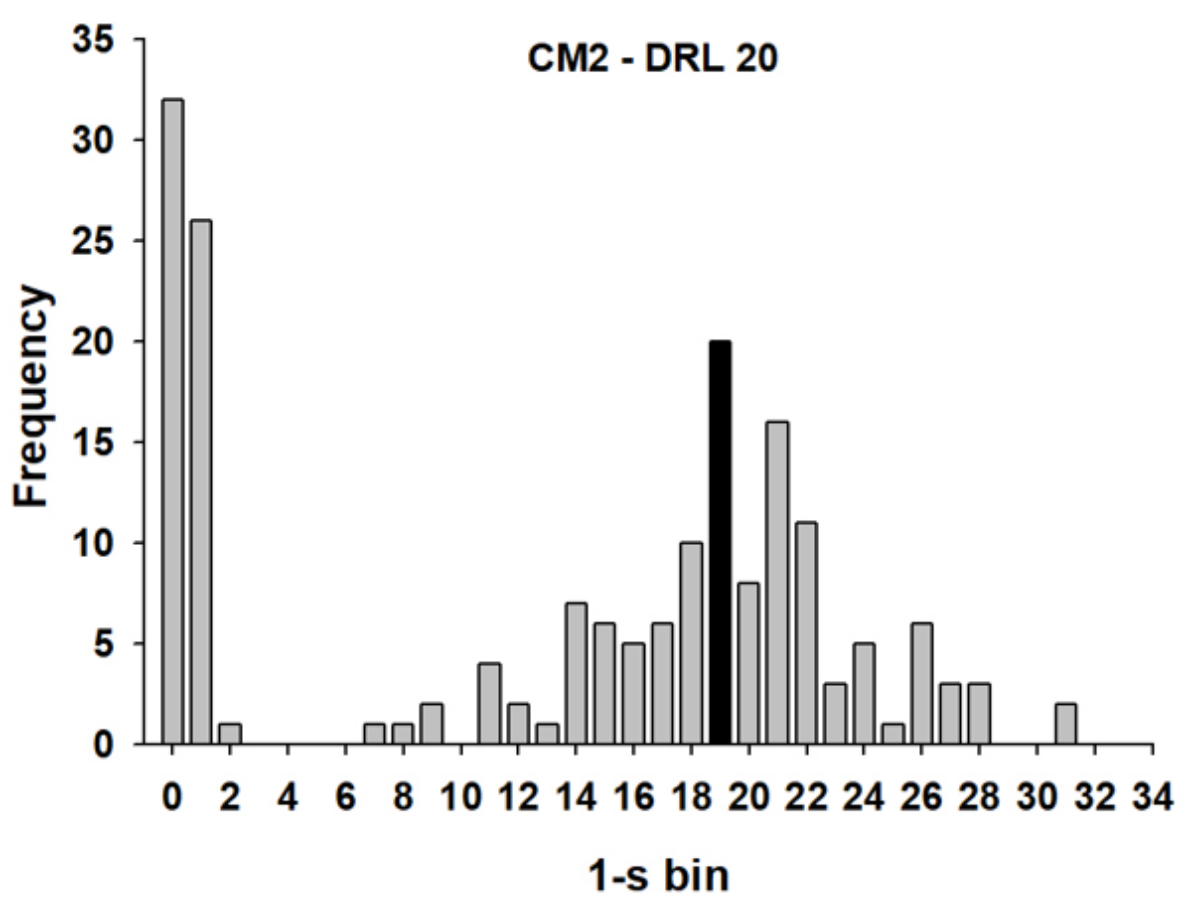

Figure C. Representative IRT histogram from a male control rat on the DRL 20-s schedule. The single filled bar indicates the modal IRT for that session (19 s). 